\title{
Growth and Structural \\ Properties of Rare Earth \\ Nitride Thin Films
}

by

Jay Chan

A thesis

submitted to the Victoria University of Wellington

in fulfilment of the

requirements for the degree of

Doctor of Philosophy

in Physics.

Victoria University of Wellington

2019 



\begin{abstract}
In this thesis aspects of the growth of rare earth nitride thin films and characterisation of the resulting structural, electronic and magnetic properties of the film are investigated. The rare earth nitrides are a class of materials which combine interesting electronic and magnetic properties with potential applications in novel spintronic device structures.

We study the formation of a preferential orientation in polycrystalline thin films of GdN deposited by electron-beam physical vapour deposition. X-ray diffraction is used to characterise the crystalline structure of films of varying thickness to identify a preference to grow along the [111] crystal direction, understood in terms of an evolutionary selection process. Variations in the film microstructure as a result of growth parameter variation are also correlated to electronic and magnetic properties.

Investigation of the epitaxial growth of $\mathrm{SmN}$ on $\mathrm{AlN}$ surfaces revealed a novel growth orientation transition, controllable only via the growth temperature. Epitaxial integration of rare earth nitrides with III-nitride surfaces has previously only resulted in (111)-oriented growth on the (0 001 ) surface as is expected from matching of the close-packed planes in the different crystal structures. High growth temperatures $\left(\geq 800^{\circ} \mathrm{C}\right)$ induce $(001)$-oriented growth of $\mathrm{SmN}$ on the same (0001) AlN surface. This unexpected cubeon-hexagon geometry is confirmed through ex situ x-ray and in situ electron diffraction, the latter for which a computational simulation tool was developed to model and understand.

The viability of using Sm as a temporary capping layer for rare earth nitride thin film samples is investigated. Capping layers are required to passivate samples due to their high reactivity, limiting the range of ex situ
\end{abstract}


characterisation techniques that can be performed on them. Elemental Sm is relatively volatile raising the possibility of removing a Sm cap in situ using moderate annealing temperatures $\left(400^{\circ} \mathrm{C}\right.$ to $\left.600{ }^{\circ} \mathrm{C}\right)$. The ability to remove the capping layer would allow in situ characterisation techniques to be performed in ultra high vacuum systems not directly connected to the growth system. In situ electron diffraction is used to characterise the growth and thermal annealing of Sm grown on top of epitaxial GdN layers, and the effects of the thermal removal process on the structural, electronic and magnetic properties of the GdN layer are investigated. 


\section{Acknowledgments}

This experimental endeavour has not been the work of one person. The combined experience from the Spintronics group at Victoria University of Wellington is what has made this thesis possible.

Thanks first to my supervisor Franck Natali, especially for his patience and understanding throughout the writing process, and the academics of the group, Ben Ruck and Joe Trodahl, who have helped my scientific development greatly while exploring a new and unfamiliar field along with all the opportunities I have been afforded along the way.

Thanks to the people I had the opportunity of meeting and working with during my time at Victoria who have all taught me something or I have learned something from, including the spintronics group (current and former members); Eva Anton, James McNulty, Luis Figueras, Chang-Min Lee, Harry Warring, Bart Ludbrook, Tanmay Maity, Natalie Plank, Simon Granville, Felicia Ullstad, Jackson Miller, Will Hewett.

A special thanks to Stéphane Vézian, Benjamin Damilano and Mohamed Al Khalfioui for their hospitality and help during my stay at Valbonne.

Thanks to my family for all the support. 


\section{Contents}

$\begin{array}{lll}1 & \text { Introduction } & 1\end{array}$

1.1 Outline . . . . . . . . . . . . . . . . . . . . . . . . 3

1.2 Attribution . . . . . . . . . . . . . . . . 3

1.3 Publications . . . . . . . . . . . . . . . . . . . . . . . . . . . . . . . 4

1.3 .1 Patents . . . . . . . . . . . . . . 4

2 Rare Earth Nitrides 5

$2.1 \quad$ History and motivation . . . . . . . . . . . . . . . . 5

$2.1 .1 \quad$ Crystal Structure . . . . . . . . . . . . . . . . . . 7

$2.1 .2 \quad$ Magnetism. . . . . . . . . . . . . . . . . . . . . 8

2.1 .3 Electronic transport $\ldots \ldots \ldots$. . . . . . . . . . 9

2.2 Thin film growth . . . . . . . . . . . . . . . . . . . 10

$2.2 .1 \quad$ Physical vapour deposition . . . . . . . . . . . . . . 11

2.2 .2 Chemical vapour deposition . . . . . . . . . . . . . . 14

2.2 .3 Sources . . . . . . . . . . . . . . . . . 14

$2.2 .4 \quad$ Substrates and capping layers . . . . . . . . . . . 15

\begin{tabular}{llr}
\hline 3 & Experimental Methods & 19
\end{tabular}

3.1 RHEED . . . . . . . . . . . . . . . . . . . . . . . . . . 20

$3.1 .1 \quad$ Crystallographic Theory . . . . . . . . . . . . . . . 21

3.1 .2 Kinematic RHEED simulation . . . . . . . . . . . . . 26

3.1 .3 RHEEDSim GUT . . . . . . . . . . . . . . . . . . . . . . 31

3.1 .4 Summary . . . . . . . . . . . . . . . . . . . . 34 
3.2 Scanning probe microscopy . . . . . . . . . . . . . . . . 35

3.3 X-ray diffraction $\ldots \ldots \ldots \ldots$. . . . . . . . . . . . . 36

3.3 .1 X-ray optics . . . . . . . . . . . . . . . . . . 36

3.3 .2 Geometry . . . . . . . . . . . . . . . . . . . 37

3.4 X-ray Reflectivity . . . . . . . . . . . . . . . . . . . . . . . 39

$3.4 .1 \quad$ XRR at VUW . . . . . . . . . . . . . . . . . . . . . . 39

3.4 .2 Example reflectivity profiles (simulated) . . . . . . . 40

3.4 .3 Multilayer systems . . . . . . . . . . . . . . . 43

3.5 Rutherford Backscattering Spectrometry . . . . . . . . . . . . 43

3.6 Cross-section Scanning Electron Microscopy . . . . . . . . . . 44

3.7 Other Characterisation Methods . . . . . . . . . . . . . . . . . 45

3.7 .1 Electrical characterisation . . . . . . . . . . . . 45

3.7 .2 Magnetic characterisation . . . . . . . . . . . . 48

4 Preferential growth of GdN 51

4.1 Introduction . . . . . . . . . . . . . . . . . . 51

4.2 Thin film growth and texture formation . . . . . . . . . . . . 53

4.3 Thin film microstructural evolution . . . . . . . . . . . . . . . 54

4.4 Sample growth and measurement . . . . . . . . . . . . . 55

4.5 Thin film thickness measurements . . . . . . . . . . . . 56

$4.5 .1 \mathrm{XRR} \ldots \ldots \ldots \ldots \ldots \ldots$

$4.5 .2 \mathrm{RBS} \ldots \ldots \ldots \ldots \ldots$

4.5 .3 SEM . . . . . . . . . . . . . . . . . . . . . . . . . . 59

4.5 .4 Tooling factor . . . . . . . . . . . . . . . . . . 59

4.6 Gd and nitrogen flux ratio . . . . . . . . . . . . . . . . . . . . 62

4.7 Thickness dependence of $\mathrm{GdN}$. . . . . . . . . . . . . . . . . 64

$4.7 .1 \mathrm{XRD} \ldots \ldots \ldots \ldots \ldots \ldots$

4.7 .2 Electrical transport . . . . . . . . . . . . . . . . . 72

4.7 .3 Magnetic properties . . . . . . . . . . . . . . . . 74

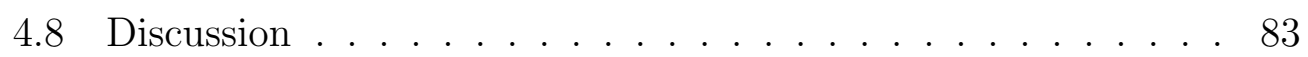

$4.8 .1 \quad$ Flux rate assumptions . . . . . . . . . . . . . . . 83

$4.8 .2 \mathrm{~N}_{2}$ dissociative adsorption $\ldots \ldots \ldots \ldots$. . . . . . 84 
4.9 Conclusion . . . . . . . . . . . . . . . . . . . . . . 85

$\begin{array}{lll}5 & \text { Epitaxial orientation transition in } \mathrm{SmN} & 87\end{array}$

5.1 Introduction . . . . . . . . . . . . . . . . . . . . . . 87

5.2 Sample growth and measurement . . . . . . . . . . . . . . . 89

5.3 RHEED . . . . . . . . . . . . . . . . . . . . . . . . . 90

$5.4 \mathrm{XRD} \ldots \ldots \ldots \ldots \ldots \ldots \ldots$

5.5 STM $\ldots \ldots \ldots \ldots 10 \ldots \ldots$

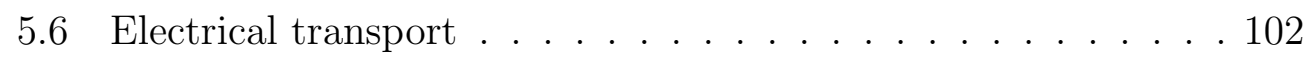

5.7 Discussion $\ldots \ldots \ldots \ldots$. . . . . . . . . . . . . . . . . . . . . . 104

5.7 .1 Epitaxial domains and growth orientation . . . . . . . 104

5.7 .2 Effect of growth temperature . . . . . . . . . . . 106

$5.7 .3 \quad$ Energy considerations . . . . . . . . . . . . . . 108

$5.7 .4 \quad$ Nitrogen precursor dependence . . . . . . . . . . . . . 110

5.7 .5 Other members of the REN series . . . . . . . . . . . . 111

5.8 Conclusion $\ldots \ldots \ldots \ldots$. . . . . . . . . . . . . . . . . . . . . . . . . . 112

$6 \quad$ Removable Samarium capping layer 113

6.1 Introduction . . . . . . . . . . . . . . . . . . . . . . . . . 113

6.2 Properties of elemental Sm . . . . . . . . . . . . . . . . 115

6.2 .1 Structure . . . . . . . . . . . . . . . . . . . 115

6.2 .2 Reactivity . . . . . . . . . . . . . . 115

6.2 .3 Sublimation of bulk Sm . . . . . . . . . . . . 116

6.3 Sample growth and measurement . . . . . . . . . . . . . . . . 118

6.4 Stability of GdN . . . . . . . . . . . . . . . . . . . . . . . . . 119

6.5 Epitaxial Sm growth on $\mathrm{GdN}$. . . . . . . . . . . . . . . . 121

6.5 .1 Structural properties . . . . . . . . . . . . . . . 121

6.5 .2 Electric and magnetic properties . . . . . . . . . . . . . 124

6.6 Thermal desorption of $\mathrm{Sm}$. . . . . . . . . . . . . . . . 125

6.6.1 Annealing without exposure to atmosphere . . . . . . . 127

6.6 .2 Sm capping layer exposed to atmosphere . . . . . . . . 133

6.7 Discussion and conclusion . . . . . . . . . . . . . . . . . . . . . 138 
6.7 .1 Samarium oxide . . . . . . . . . . . . . . . . . . . 138

6.7 .2 Annealing time . . . . . . . . . . . . . . 140

6.7 .3 Conclusion . . . . . . . . . . . . . . . . . 141

$\begin{array}{lll}7 & \text { Summary and outlook } & 143\end{array}$

7.1 Summary . . . . . . . . . . . . . . . . . . . . . . . . . . . 143

7.2 Future work . . . . . . . . . . . . . . . . . . . . . . . . . . . . 144 


\section{List of abbreviations}

AFM Atomic Force Microscopy

ARPES Angle Resolved Photoemission Spectroscopy

CNRS-CRHEA Centre national de la recherche scientifique - Centre de Recherche sur l'Hétéro-Epitaxie et ses Applications

CVD Chemical Vapour Deposition

DHCP Double Hexagonal Close Packed

DRC Deposition Rate Controller

EB Electron Beam

FC Field-cooled

FCC Face Centered Cubic

FWHM Full Width at Half Maximum

GIXRD Grazing Incidence X-ray Diffraction

HCP Hexagonal Close Packed

HRXRD High Resolution X-ray Diffraction

LED Light Emitting Diode

MBE Molecular Beam Epitaxy

PLD Pulsed Laser Deposition

PVD Physical Vapour Deposition

RAM Random Access Memory

RBS Rutherford Backscattering Spectrometry 
RE Rare Earth

REN Rare Earth Nitride

RF Radio Frequency

RHEED Reflection High Energy Electron Diffraction

SEM Scanning Electron Microscopy

SQUID Superconducting Quantum Interference Device

STM Scanning Tunneling Microscopy

SZM Structure Zone Model

TCR Temperature Coefficient of Resistivity

UHV Ultra High Vacuum

$\mathbf{V}_{N}$ Nitrogen Vacancy

VSM Vibrating Sample Magnetometry

VUW Victoria University of Wellington

W-H Williamson-Hall

XPS X-ray Photoelectron Spectroscopy

XRD X-ray Diffraction

XRR X-ray Reflectivity

YSZ Yttrium Stabilized Zirconium

ZFC Zero-field Cooled 


\section{Chapter 1}

\section{Introduction}

Semiconductor technology forms the basis for electronics used in everyday life, with semiconductor devices such as diodes and transistors used to manipulate the flow of electric charge in the form of electrons to process signals and information. The field of spintronics adds onto conventional electronics by additionally manipulating the magnetic property of the electron, its spin. Metal-based spintronics has had a huge technological impact; for example the read heads in modern hard disk drives use giant magnetoresistance to sense smaller and smaller magnetic fields enabling the huge increases in storage capacity [1]. A merging of semiconductor and magnetic technologies has potential advantages over conventional electronics; non-volatility, increased data processing and storage speed combined with decreased power consumption [2 4]. However, there is a significant challenge in filling this gap for semiconductor-based spintronics - the requirement for new materials that combine magnetic and semiconducting properties, an exceedingly rare combination that is essential for the injection and detection of spin-polarised currents.

Recent research into the rare earth nitrides (RENs) was first motivated by the theoretical prediction that some members of the series could form ferromagnetic half-metals with near complete spin polarisation [5]. The first experimental investigations of the RENs in the mid and late 20th century 
revealed with some certainty that almost all members were ferromagnetic, adopting magnetic order at cryogenic temperatures. The electronic transport properties were however much less certain, with conflicting reports ranging from insulating to metallic for nominally identical materials, mainly due to the difficulty in producing high-quality, stoichiometric samples [6]. Only in recent years have modern advances in thin film growth technology overcome these difficulties allowing experimental characterisation to be compared to theoretical predictions and it is now widely accepted that many members of the series are semiconducting in nature [7].

As materials that intrinsically combine ferromagnetism and semiconducting behaviour, the RENs have the potential to fill the long-standing gap impeding the realisation of semiconducting spintronics. Intrinsic ferromagnetic semiconductors contrast with dilute magnetic semiconductors, where a nonmagnetic semiconductor is doped with transition metal ions such as Mn to induce ferromagnetism alongside the semiconducting behaviour while in the RENs, every rare earth ion is magnetic.

A wide range of electronic and magnetic behaviours are observed across the REN series which is especially appealing from an applied device perspective. The rare earths comprise the elements with atomic number ranging from $57(\mathrm{La})$ to $71(\mathrm{Lu})$ along which the $4 f$ orbital is filled. The spin and orbital motion of these $4 f$ electrons results in the following examples: a net magnetisation in direct opposition to the spin moments in the case of $\mathrm{NdN}[8]$; ferromagnetically aligned spins accompanied by a near-zero net magnetisation in $\mathrm{SmN}$ [9]; and a large purely spin moment with no orbital contribution in $\operatorname{GdN}[10]$. These contrasting magnetic behaviours have potential to be combined in memory element applications (non-volatile RAM).

Experimental research into the RENs is based on the fabrication and characterisation of thin film samples. Furthering the understanding of the growth process and the resulting film properties is a crucial step towards their integration into semiconductor-based spintronic devices. 


\subsection{Outline}

The work in this thesis focuses primarily on aspects of the growth process of REN thin films and structural characterisation techniques, and relating these to the resulting electrical and magnetic properties. The thesis is divided into chapters as follows:

- Chapter 2 briefly reviews the current understanding of properties of the rare earth nitrides and an overview of the thin film deposition techniques.

- Chapter 3 focuses on the experimental characterisation techniques used and developed over the duration of this thesis.

- Chapter 4 presents results demonstrating the emergence of a preferential orientation during deposition of polycrystalline GdN and some electrical and magnetic characterisation for varying growth parameters.

- Chapter 5 presents results on the surprising epitaxial growth orientation of $\mathrm{SmN}$ on AlN controllable by the growth temperature and subsequent structural and electrical sample characterisation.

- Chapter 6 presents preliminary work on using Sm as a temporary capping layer for GdN samples, intended for characterisation techniques requiring access to the GdN surface.

\section{$1.2 \quad$ Attribution}

The following experimental work was done in collaboration with others:

- Chapter 3: Development of the XRR technique done with O. Pantoja

- Chapter 4: RBS measurements and analysis performed by P. Murmu 
- Chapter 5: SmN sample growth carried out by S. Vèzian, electrical characterisation assisted by M. Al Khalfioui

- Chapter 6: AFM measurements primarily carried out by M. Le Ster

\subsection{Publications}

1. F. Ullstad, J. R. Chan, H. Warring, N. Plank, B. Ruck, J. Trodahl, and F. Natali. "Ohmic contacts of $\mathrm{Au}$ and $\mathrm{Ag}$ metals to n-type GdN thin films". AIMS Materials Science 2.2 (2015), pp. 79-85

2. J. R. Chan, S. Vézian, J. Trodahl, M. A. Khalfioui, B. Damilano, and F. Natali. "Temperature-Induced Four-Fold-on-Six-Fold Symmetric Heteroepitaxy, Rocksalt SmN on Hexagonal AlN". Crystal Growth 86 Design 16.11 (2016), pp. 6454-6460

3. J. R. Chan, M. Al Khalfioui, S. Vézian, J. Trodahl, B. Damilano, and F. Natali. "Effect of the growth temperature and nitrogen precursor on the structural and electrical transport properties of SmN thin films". MRS Advances 2.3 (2017), pp. 165-171

\subsubsection{Patents}

1. F. Natali, B. J. Ruck, J. H. Trodahl, and J. R. Chan. "Rare Earth Nitride Structures and Devices and Method for Removing a Passivating Capping". European Patent 16202663.7 (Filed 07 December 2016)

2. F. Natali, S. Vézian, J. R. Chan, B. J. Ruck, and J. H. Trodahl. "Rare Earth Nitride Structure or Device and Fabrication Method". US Patent No. 15/607,596 (Filed 29 May 2017) 


\section{Chapter 2}

\section{Rare Earth Nitrides}

\subsection{History and motivation}

The rare earths comprise the elements with atomic number ranging from 57 (lanthanum) to 71 (lutetium). It is across this series that the $4 f$ orbitals are filled, giving the rare earths and their compounds a wide range of interesting magnetic properties with as many as 7 unpaired electrons in the $4 f$ orbital in the case of Gd. Attempts to synthesise the rare earth nitrides (RENs) and study their properties began in the 1950s after techniques to purify rare earth sources were developed 16$]$. Although the magnetic character of the REN series was identified early on, with many members adopting magnetic order at cryogenic temperatures below $70 \mathrm{~K}$, characterisation of the electronic transport properties was much less certain with the difficulties in creating stoichiometric samples due to their high reactivity [6]. The methods used to synthesise bulk crystals, by reacting rare earths in various forms directly with nitrogen or ammonia were prone to contamination as well as high concentrations of nitrogen vacancies 17, 18. Even small deviations from one-to-one stoichiometry, i.e. samples of $\mathrm{RE}_{1} \mathrm{~N}_{1-x}$ for small $x$ can produce samples ranging from insulating to metallic conducting behaviour for nominally identical compounds, while not significantly affecting the magnetic ordering. Interest in the RENs recovered in the early 2000s 
with theoretical predictions of interesting conductive states across the series such as semiconducting, semimetallic and even half metallic states $[5]$. Combined with the magnetic behaviour known from the early studies, the RENs would demonstrate properties ideal for exploitation in semiconductor-based spintronic technologies [3].

Rather than concentrating on synthesising bulk crystals as in the early studies, thin films of RENs (ranging from tens of monolayers up to hundreds of nanometres in thickness) have been the focus of most modern research [7]. Development of the technologies for thin film growth have been spurred on in recent decades by the ever-increasing demands of the semiconductor industry, and in particular ultra high vacuum based techniques have been used to address the earlier problems with REN synthesis. Deposition of the films in vacuum chambers with base pressures a billionth of atmospheric pressure limits the contamination from unwanted species. A relatively simple reactive physical vapour deposition based process could be used to synthesise many of the RENs; a pure RE metal source is evaporated in a low partial pressure of $\mathrm{N}_{2}$ and allowed to condense on an appropriate substrate, with a spontaneous reaction occurring to form the REN thin film. To prevent the oxidation that plagued early samples, passivating capping layers using appropriate materials could then be deposited on the REN thin film to prevent them reacting with ambient atmosphere.

The study of REN thin films synthesised in this way or by using similar techniques has shed light on many of the questions surrounding the electronic state and REN thin films are primarily semiconducting, with intrinsic doping from nitrogen vacancies formed during the deposition process. While answering some questions, the thin film deposition process has unleashed many more, surrounding how varying the growth parameters can influence the electronic and magnetic properties, such as enhancement of the magnetic transition temperature from $70 \mathrm{~K}$ to $200 \mathrm{~K}$ in N-deficient GdN [19]. Control of the film properties would be essential for any integration into device structures whose functionality relies on reproducibility and reliability. 


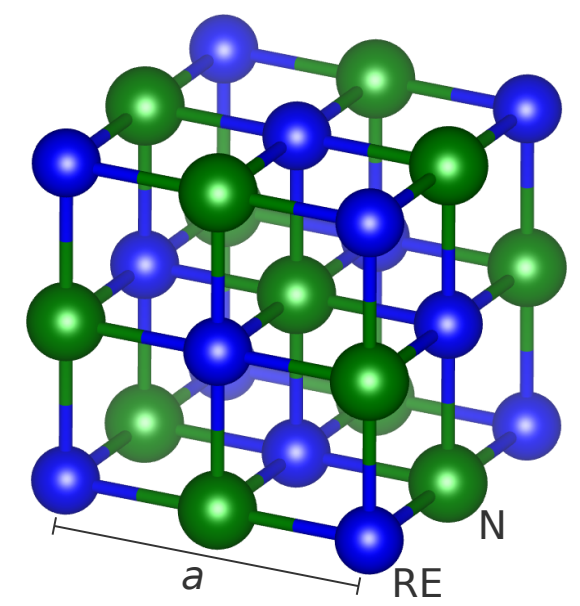

Figure 2.1: The rock salt structure assumed by the RENs. The RE ${ }^{3+}$ cation and $\mathrm{N}^{3-}$ anion form separate face-centred cubic lattices translationally offset by half of the lattice constant $a$.

Despite the recent progress, no reliable experimental data for many fundamental material properties of most of the RENs, either bulk or thin film, including thermal properties such as thermal expansion and thermal conductivity, and elastic moduli (e.g. bulk modulus and Young's modulus) have been reported; the main limitation being their reactivity and thus the necessity for passivating capping layers on thin film samples. Such properties are of particular importance in understanding the epitaxial growth of REN thin films and the formation of defects, which require growth temperatures several hundred degrees above room temperature. Additionally, the nature of passivating capping layers precludes many characterisation techniques that could otherwise be used to explore the properties of the thin films.

\subsubsection{Crystal Structure}

The RENs all crystallise in the cubic rock salt structure (space group Fm $\overline{3} \mathrm{~m}$ ) in a one-to-one stoichiometric ratio between the rare earth and nitrogen (Fig. 2.1). The rare earths adopt a trivalent state except $\mathrm{CeN}$ which exhibits a mixed trivalent and tetravalent state. The lattice parameters de- 


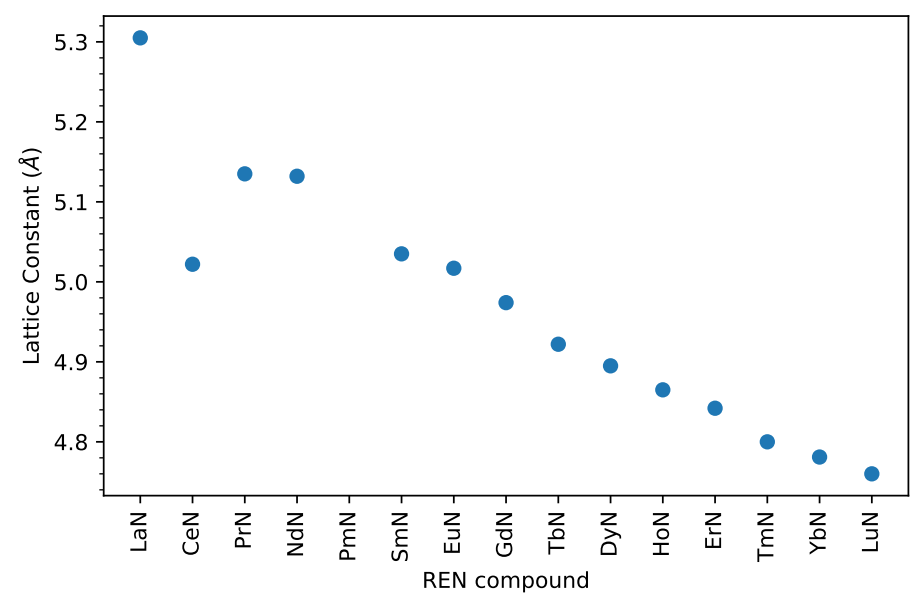

Figure 2.2: Experimental lattice constants for members of the REN series [7]. The lattice constants decrease along the series as the RE cation radius decreases.

crease across the series with increasing atomic number (Fig. 2.2) as the ionic radius decreases $[7]$. One aspect important for technological applications is the potential to grow multilayer structures consisting of different members of the REN series. The small lattice mismatch of less than $1 \%$ between most neighbouring members and a total difference of $10 \%$ across the series raises the possibility of epitaxial growth of such structures. The simple rock salt structure common to all members makes them somewhat amenable to theoretical calculations which can then be compared to experiment.

\subsubsection{Magnetism}

Early investigations into the magnetic ordering of members of the REN series found a majority were ferromagnetic: NdN, GdN, TbN, DyN, HoN, ErN, with some antiferromagnetic: $\mathrm{SmN}$ and $\mathrm{YbN}[16,20]$. Recent investigations into thin films have reconfirmed most of these observations, except for $\mathrm{SmN}$ which has been reidentified as being ferromagnetic with a nearzero net magnetic moment with a Curie temperature of about $30 \mathrm{~K}[9]$. The 
most thoroughly studied member is GdN, mainly because it has the highest Curie temperature typically reported to be between $50 \mathrm{~K}$ to $70 \mathrm{~K}$, but it notably also has a large, purely spin magnetic moment of $\sim 7 \mu_{B} / \mathrm{Gd}^{3+}$ from the half-filled $4 f$ shell of $\mathrm{Gd}^{3+}$. The stoichiometry can have a notable effect on the Curie temperature as seen in the cases of GdN 19 and $\operatorname{EuN} 21$ where enhanced Curie temperatures of up to $200 \mathrm{~K}$ and $120 \mathrm{~K}$ are reported respectively. The formation of magnetic polarons around nitrogen vacancies in GdN has been suggested as the origin of the interplay between magnetism and charge transport 22 .

\subsubsection{Electronic transport}

Experimental work on REN thin films in recent years was sparked by the theoretical predictions that some of them may show interesting conducting states such as GdN possibly being half-metallic [5]. The combination of many results have been used to demonstrate the intrinsic semiconducting nature. Here we will mainly focus on most studied member so far, GdN, which has the most supporting experimental evidence.

The largest impediment to experimental investigations of the electronic transport in the RENs is their reactivity and the difficulty of producing samples with good stoichiometry. Doping via nitrogen vacancies has been shown to be the source of the observed n-type conductivity in GdN [23] and the high carrier concentrations on the order of $\sim 10^{20} \mathrm{~cm}^{-} 3$. A quick calculation to estimate the order of magnitude of nitrogen vacancies to result in this carrier concentration, assuming each vacancy contributes 1 electron to the conduction band gives $\sim 1 \%$ of the $\sim 3 \times 10^{22} \mathrm{~cm}^{-3}$ nitrogen sites to be vacant. Such high carrier concentrations with accompanying low resistivities are often measured in epitaxial films in particular, as the high growth temperatures dictate the formation of nitrogen vacancy concentrations on that level 24, 25].

The temperature coefficient of resistivity (TCR), i.e. the slope of the resistivity measured as a function of temperature in these samples is typi- 
cally positive; behaviour that is often considered to be metallic, but is also seen in degenerately doped semiconductors. Polycrystalline thin film samples intentionally grown to have low nitrogen vacancy concentrations show much higher resistivities and negative TCR, more characteristic of an intrinsic semiconductor [19]. Perhaps the most convincing experimental evidence for semiconducting behaviour is the ability to dope epitaxial GdN with $\mathrm{Mg}$ whereby $\mathrm{Mg}$ concentrations of up to $5 \times 10^{19} \mathrm{~cm}^{-3}$ are able to compensate for the inherent doping from nitrogen vacancies resulting in carrier concentrations of $10^{16} \mathrm{~cm}^{-3}$ and resistivities as high as $10^{3} \Omega \cdot \mathrm{cm}[26]$. The Curie temperature is additionally observed to shift from $70 \mathrm{~K}$ to $\sim 50 \mathrm{~K}$, in agreement with the magnetic polaron situation suggested before [22].

Measurements of the optical band gap in the RENs have also been used to show their semiconducting nature. The optical band gap has been measured for polycrystalline $\mathrm{GdN}$ to be $1.31 \mathrm{eV}$ [27], and indirect and direct transitions found to be $0.95 \mathrm{eV}$ and $1.18 \mathrm{eV}$ respectively in epitaxial GdN [28]. The optical band gap has also been measured in DyN to be $1.2 \mathrm{eV}$ [29], EuN with a gap of $0.9 \mathrm{eV}[30]$, NdN with a gap of $0.9 \mathrm{eV}[8]$ and $\mathrm{YbN}$ with a gap of $1.5 \mathrm{eV} 31$.

\subsection{Thin film growth}

The advancement in ultra-high vacuum based film deposition techniques has been crucial in obtaining high quality REN samples for experimental characterisation. Physical vapour deposition techniques, in particular molecular beam epitaxy, are the main techniques employed for the growth of all samples explored in this thesis. Other techniques that have been applied to REN thin film growth are pulsed laser deposition and radio-frequency sputter deposition and very recently chemical vapour deposition. The main features of each deposition technique and specific details related to REN deposition are described below. 


\subsubsection{Physical vapour deposition}

Physical vapour deposition (PVD) broadly encompasses a number of vacuum deposition techniques used to create thin films and coatings. The specific methods mainly differ by the method in which solid sources are forced into a vapour phase which then condenses onto a substrate in the form of a thin film. Solid source can be evaporated thermally, i.e. using resistive heating (evaporative deposition) or by bombarding the source with an electron beam (EB-PVD). Pulsed laser deposition uses high-powered pulsed lasers targeted at the source to vaporise it. Direct current sputtering involves bombarding the target material with energised gas ions to sputter atoms onto the substrate. With regards to the growth of thin films, each technique has advantages and disadvantages such as the materials that a technique can be applied to, control of the deposition parameters such as the deposition rate, and complexity of the technique and the required hardware and equipment.

High vacuum deposition chambers are used to limit the incorporation of impurities into the film during deposition and in the case of RENs, to prevent reaction of the deposited REN film with atmosphere. Additionally, high vacuum ensures that the mean free path of the source vapour is generally larger than the source-to-sample distance, meaning that deposition is line-ofsight from the source to the sample. An example schematic of an ultra high vacuum deposition system is shown in Fig. 2.3. A separately pumped load lock chamber is used for loading and transferring samples without breaking the vacuum in the main deposition chamber. Substrates are loaded onto a manipulator which can additionally heat the substrate for outgassing or high temperature deposition. Solid sources are evaporated from the electron gun source, thermally via resistive heating or using an effusion cell while $\mathrm{N}_{2}$ can be introduced through a leak valve with a controlled mass flow. The deposition conditions are monitored using the quartz crystal microbalance for the deposition rate while the background pressure recorded by the vacuum gauge. A mass spectrometer can be used to identify the residual gas composition. Deposition can also be monitored in situ using reflection high energy 


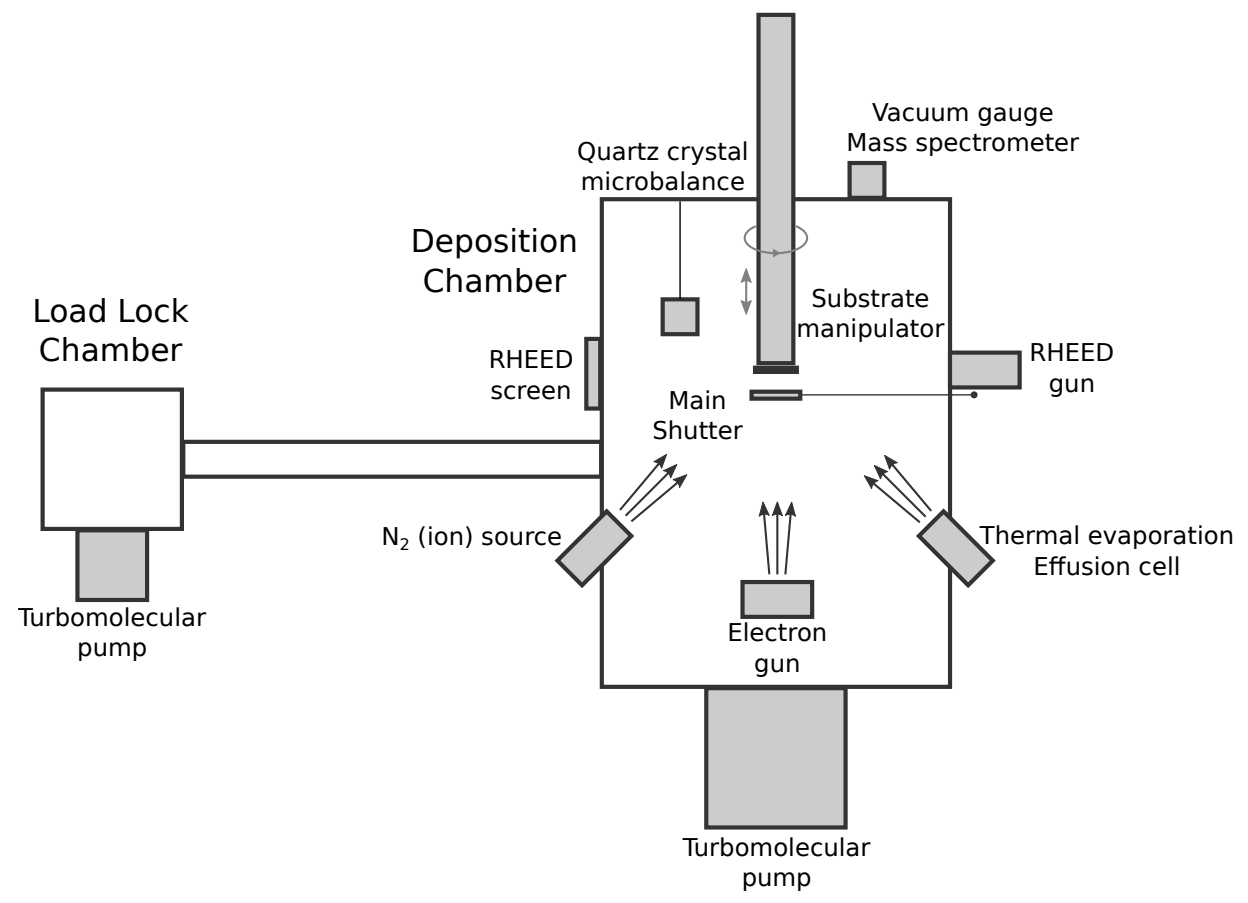

Figure 2.3: Schematic diagram showing the main components and rough layout of a ultra high vacuum deposition system. 
electron diffraction (RHEED) employing a grazing incidence geometry.

\section{Epitaxial growth and molecular beam epitaxy}

Epitaxy is a term used to refer to the growth of ordered crystalline films or layers on top of crystal substrates. The quality of the deposited epitaxial layer and its resulting properties depends on the choice of substrate material and the growth conditions and processes. Epitaxy can be split into two general categories: homoepitaxy, where the substrate and epitaxial layer are a single identical material, and heteroepitaxy, where the substrate and epitaxial layer are different materials. The growth of rare earth nitride thin films all fall under heteroepitaxy as no native rare earth nitride substrates exist. In general, heteroepitaxy allows a wide range of materials to be grown on different substrates or for heterostructures to be grown consisting of multiple epitaxial layers of different materials. Naturally, epitaxy of layers of different materials results in films of lower quality than could be obtained through homoepitaxy; one of the main goals of epitaxy in general is to explore the rich parameter space to optimise growth processes and to grow structures of adequate quality for particular applications.

Of the RENs, epitaxial thin film growth has been demonstrated only for CeN [32], GdN [24, 25, 33 36], SmN [25, 37] and EuN [25]. The molecular beam epitaxy (MBE) technique has so far been shown to be able to produce the highest quality REN thin film samples. In the technique, a molecular beam is created using one of the evaporation techniques as above to be directed towards a chosen substrate. The key advantages of MBE that lends itself particularly well to the deposition of RENs is the control of the deposition rate $\left(300 \mathrm{~nm} \mathrm{~h}^{-1}\right.$ or lower) that allows epitaxial growth of the film. The substrate is normally heated to several hundred degrees to allow deposited adatoms to diffuse and nucleate on the surface in a highly ordered fashion. 


\subsubsection{Chemical vapour deposition}

Although not explored in this thesis, chemical vapour deposition (CVD) is another process often used industrially to produce thin film materials. In contrast to PVD, a chemical process is used, where volatile precursors are allowed to react or decompose at the substrate surface to form the thin film of the desired composition. The difficulty with applying CVD to the growth of RENs has been finding efficient RE precursors. The deposition of GdN and DyN via metal-organic CVD has been demonstrated using guanidinato and amidinate complexes of Gd and Dy 38,39 .

\subsubsection{Sources}

The precursor sources for the thin film samples grown and analysed in this thesis (GdN, SmN, Sm, and GaN/AlN capping layers) are detailed. Rare earth metal sources are cleaned with organic solvents and heated up and outgassed inside the vacuum chamber to remove residual solvents. Electron beam evaporation is used to evaporate Gd which has a high melting point of $1312^{\circ} \mathrm{C}$ while resistive heating can be used to evaporate $\mathrm{Sm}$ which has a lower melting point of $1072{ }^{\circ} \mathrm{C}$ and a much higher vapour pressure at lower temperatures. Ga used for the capping of some samples was also evaporated via electron beam. Effusion cells were also employed for the evaporation of $\mathrm{Al}$, and for Sm and Ga.

There are a number of choices for the nitrogen precursor for the growth of RENs. For some (including GdN and $\mathrm{SmN}$ ) it is possible to use pure molecular $\mathrm{N}_{2}$ which catalytically reacts with the rare earth deposited on the substrate to form the REN. $\mathrm{NH}_{3}$ has also been used as a nitrogen precursor in epitaxial growth, relying on the thermal cracking of $\mathrm{NH}_{3}$ to provide access to atomic N. Alternatively, activated nitrogen sources can been used such as plasma or Kaufman ion sources when radical nitrogen is required. 


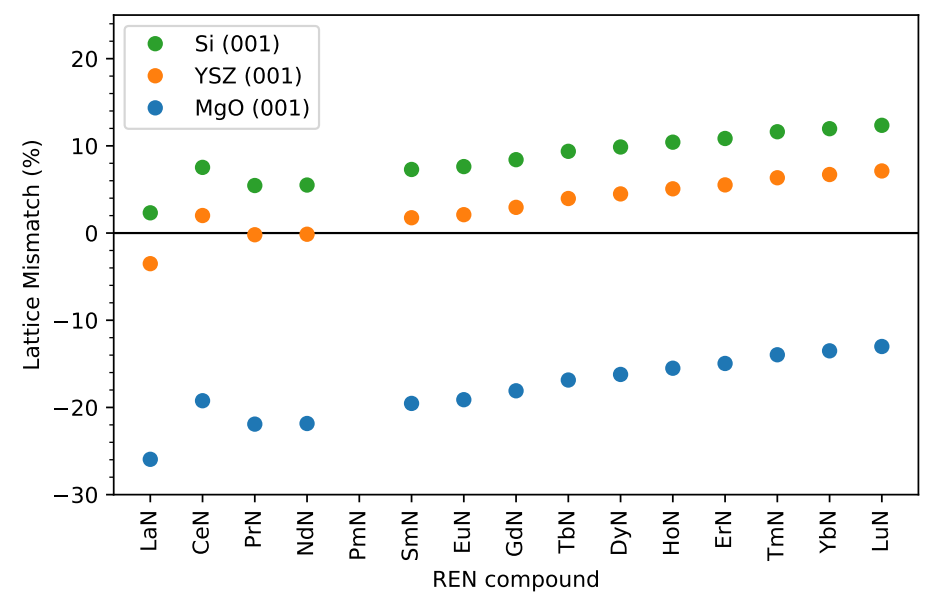

Figure 2.4: Calculated lattice mismatch between the (001) RENs

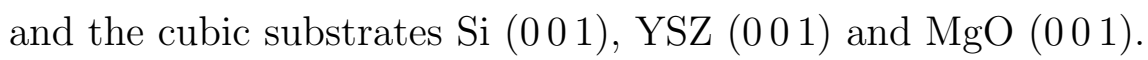

\subsubsection{Substrates and capping layers}

A number of different substrates have been used for the growth of polycrystalline REN thin films, such as $\mathrm{Al}_{2} \mathrm{O}_{3}$ and $\mathrm{Si}$ or amorphous substrates like fused silica, which the RENs adhere to well [7]. Choosing suitable substrates for epitaxial growth is more challenging, due to the lattice mismatch of the RENs with common candidates. The epitaxial lattice mismatch $f$ defined as

$$
f=\frac{a_{s u b}-a_{e p i}}{a_{\text {sub }}}
$$

where $a_{\text {sub }}$ and $a_{e p i}$ are the reference lattice constants of the substrate and epitaxial layer respectively. For $f<0$ the epitaxial layer would be under compressive strain and for $f>0$, tensile strain is expected. The lattice mismatch is shown for the cubic materials $\mathrm{Si}(a=5.431 \AA)$, YSZ $(a=5.125 \AA)$ and $\mathrm{MgO}$ $(a=4.212 \AA)$ in Fig. 2.4. The nearest lattice-matched cubic substrate is YSZ, although an oxide layer forms at the YSZ-REN interface due to the oxygen ion mobility in the substrate [35]. Si has a relatively low lattice mismatch but it too reacts with the depositing $\mathrm{RE}$ at high temperatures to form rare earth silicides. $\mathrm{MgO}$, also cubic, has been used for epitaxial growth but the 


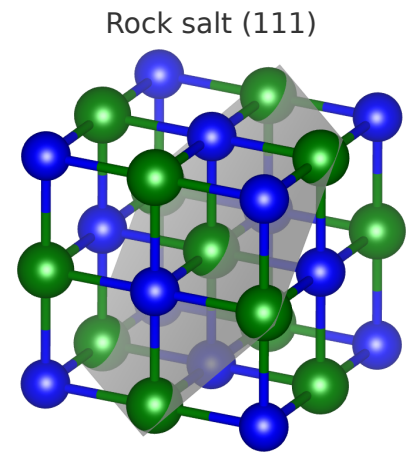

Figure 2.5: Comparison of the rock salt structure of the RENs with the wurtzite structure of the III-nitrides (AlN, GaN, InN). The matching of the rock salt (111) plane and wurtzite (0001) plane is highlighted.

lattice mismatch with the RENs is very high [33], more than $-18 \%$ in the case of GdN.

As an alternative to cubic substrates, the epitaxial growth of the cubic RENs on hexagonal wurtzite GaN and AlN has been demonstrated 24, 25, 34, 36], taking advantage of matching of the close-packed (111) plane of the REN with the ( $\left.\begin{array}{llll}0 & 0 & 0 & 1\end{array}\right)$ plane of the wurtzite structure (Fig. 2.5). The IIInitride is grown epitaxially on $\mathrm{Si}$ or $\mathrm{Al}_{2} \mathrm{O}_{3}$ as a buffer layer for the REN layer deposited on top. Such systems also have a moderately high lattice mismatch ( $-11 \%$ for the case of GdN (111) on GaN (0001), see Fig. 2.6), though the potential to combine the functionalities of the RENs with the wide band gap III-nitrides is compelling. The III-nitrides have many applications in optoelectronics (LEDs), high-power and high-frequency devices [40]. Notably, the lattice mismatch with $\mathrm{InN}$ is nearly zero for the middle of the REN series however InN substrates or templates are not available commercially.

Capping layers are required to passivate the REN thin films due to their reaction with the atmosphere if exposed. A number of different capping layer materials have been used depending subsequent measurements to be performed on the sample[7]; metallic layers such as $\mathrm{NbN}$ are suitable as 


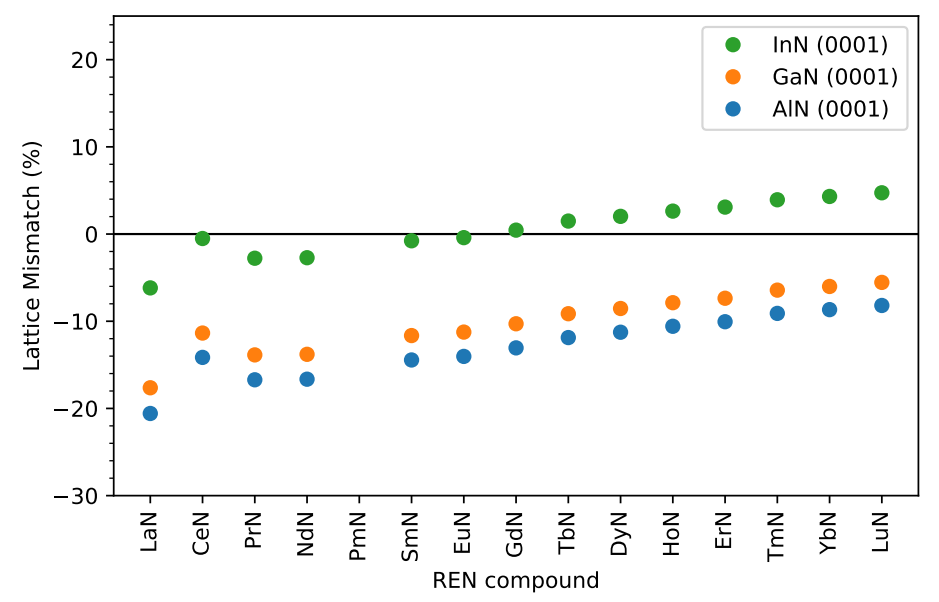

Figure 2.6: Calculated lattice mismatch between (111)-oriented RENs and the (0 0001 ) plane of the wurtzite III-nitrides AlN GaN and $\mathrm{InN}$

top contacts for vertical transport measurements, or transparent insulating layers such as amorphous or nanocrystalline GaN or AlN which can be used for optical measurements and horizontal transport. Mainly GaN and AlN have been used in this thesis due to their good chemical stability over time. 


\section{Chapter 3}

\section{Experimental Methods}

The development of in situ and ex situ characterisation methods has been closely associated with the continuing progress of understanding and controlling the epitaxial growth process. Using such methods that will be described below is essential for gaining a better understanding of the RENs. A lacking aspect in the characterisation tools and methods for the RENs has been related to structural, surface and material properties. As mentioned earlier, reaearch groups involved in the study of RENs have focused mostly on their electronic and magnetic properties. To get a better understanding of the epitaxial growth of the RENs, one of the goals of my thesis was to further develop related experimental techniques and simulation tools that were initially unavailable at VUW or are not easy to access within NZ. The first of these techniques was RHEED, where simulation tools were developed to aid in understanding experimentally obtained diffraction patterns. The second technique focused on extending the XRD measurement capabilities at VUW with techniques more applicable to thin film samples, in particular XRR, a non-destructive technique that can be used to infer layer thicknesses. 


\subsection{RHEED}

Reflection High Energy Electron Diffraction (RHEED) is a crystallographic technique used to characterise the surface of materials. A beam of high energy electrons $(>10 \mathrm{keV})$ is directed at the surface of a sample at grazing incidence, typically less than a few degrees. Electrons interact strongly with the surface atoms on the sample and this is combined with the grazing incidence to avoid bulk interactions, enabling surface sensitivity.

The main application of RHEED is for monitoring the epitaxial growth of thin films as the grazing incidence doesn't interfere with the deposition process. Depending on the nature of the surface being analysed, a variety of characteristic RHEED patterns can be observed such as diffraction streaks resulting from a flat surface with out-of-phase domains, to transmission spots if the surface is rough with 3D islands structures. Non-epitaxial growth resulting in polycrystalline surfaces can result in diffraction arcs from the random orientation of crystallies instead. Although these patterns can still be interesting, analysis of these patterns is beyond the scope of this thesis.

While the above-mentioned qualitative observations can be quickly deduced, a quantitative description of electron diffraction from crystalline materials allows much more information to be extracted from experimental RHEED patterns. As part of this thesis project, I have developed and written a program to simulate the resulting RHEED patterns from arbitrarily oriented crystal structures to be used in qualitative analysis of experimentally obtained patterms. We develop the necessary background theory describing the diffraction of waves in the general case from 3D crystal lattices and how this is implemented algorithmically in the simulation program.

\section{D RHEED simulation}

While the following theory will focus on the general case of 3D diffraction, it is relatively simple to modify the calculation to reproduce the surface sensitivity of RHEED. Instead of defining a full 3D crystal lattice, a 2D lattice 
corresponding to the surface layers of the crystal is defined and the analysis can be continued in the same manner, ignoring the diffraction condition in the direction perpendicular to the sample.

\subsubsection{Crystallographic Theory}

\section{Crystal structure}

A crystal is an arrangement of atoms or molecules which form an ordered, periodically repeating microscopic structure. The arrangement of these atoms can be described mathematically by a lattice structure; a Bravais lattice in $n$ dimensions is generated from a set of primitive vectors $\mathbf{a}_{\mathbf{i}}$ which span $\mathbb{R}^{n}$ and consists of all points $\mathbf{R}$ where

$$
\mathbf{R}=\sum_{i=1}^{n} k_{i} \mathbf{a}_{\mathbf{i}}
$$

for $k_{i} \in \mathbb{Z}$. It is possible to specify any $3 \mathrm{D}$ Bravais lattice $\left(\mathbf{a}_{\mathbf{1}}, \mathbf{a}_{\mathbf{2}}, \mathbf{a}_{\mathbf{3}}\right)$ using 6 parameters, the lengths of the three primitive vectors $a, b, c$ and the angles between each pair of vectors, $\alpha, \beta, \gamma$. The following transformation matrix $\mathbf{T}$ describes the Bravais lattice with the columns corresponding to the primitive vectors:

$$
\mathbf{T}=\left[\begin{array}{l}
\mathbf{a}_{\mathbf{1}} \\
\mathbf{a}_{2} \\
\mathbf{a}_{3}
\end{array}\right]^{T}=\left[\begin{array}{ccc}
a & b \cos \gamma & c \cos \beta \\
0 & b \sin \gamma & c\left(\frac{\cos \alpha-\cos \beta \cos \gamma}{\sin \gamma}\right) \\
0 & 0 & c \sqrt{1-\frac{\cos ^{2} \alpha+\cos ^{2} \beta-2 \cos \alpha \cos \beta \cos \gamma}{\sin ^{2} \gamma}}
\end{array}\right]
$$

A physical crystal consists of an atomic basis, an arrangement of one or more atoms, which repeats at each lattice point $\mathbf{R}$. The location of a basis atom, $\mathbf{r}$ within a unit cell can be described by fractional coordinates $\mathbf{r}_{\text {frac }} \in \mathbb{R}^{3}$, e.g.

$$
\mathbf{r}=\mathbf{T r}_{\text {frac }}=\mathbf{T}\left[\begin{array}{l}
r_{1_{\text {frac }}} \\
r_{2_{\text {frac }}} \\
r_{3_{\text {frac }}}
\end{array}\right]
$$


where $0 \leq r_{i_{\text {frac }}} \leq 1$. As an example, a rock salt structure can be described using a cubic lattice, e.g. $a=b=c$ and $\alpha=\beta=\gamma=\frac{\pi}{2}$ giving

$$
\mathbf{T}=\left[\begin{array}{lll}
a & 0 & 0 \\
0 & a & 0 \\
0 & 0 & a
\end{array}\right]
$$

where $a$ is the cubic lattice constant and a two element basis $\left(e_{1}, e_{2}\right)$ with eight atoms,

$$
e_{1}=\left\{\left[\begin{array}{l}
0 \\
0 \\
0
\end{array}\right],\left[\begin{array}{c}
\frac{1}{2} \\
\frac{1}{2} \\
0
\end{array}\right],\left[\begin{array}{c}
\frac{1}{2} \\
0 \\
\frac{1}{2}
\end{array}\right],\left[\begin{array}{c}
0 \\
\frac{1}{2} \\
\frac{1}{2}
\end{array}\right]\right\}, e_{2}=\left\{\left[\begin{array}{c}
\frac{1}{2} \\
0 \\
0
\end{array}\right],\left[\begin{array}{c}
0 \\
\frac{1}{2} \\
0
\end{array}\right],\left[\begin{array}{c}
0 \\
0 \\
\frac{1}{2}
\end{array}\right],\left[\begin{array}{c}
\frac{1}{2} \\
\frac{1}{2} \\
\frac{1}{2}
\end{array}\right]\right\}
$$

\section{Reciprocal Lattice}

The reciprocal lattice is calculated from the Fourier transform of the crystal lattice. Instead of looking at the physical atoms in real space, we take advantage of the periodicity of the crystal; any property $f$ of the crystal that is periodic satisfies

$$
f(\mathbf{r})=f(\mathbf{R}+\mathbf{r})
$$

for any lattice point $\mathbf{R}$. The Fourier expansion of this is

$$
f(\mathbf{R}+\mathbf{r})=\sum_{m} f_{m} e^{i \mathbf{G}_{\mathbf{m}} \cdot \mathbf{r}} e^{i \mathbf{G}_{\mathbf{m}} \cdot \mathbf{R}}
$$

Noting that $\mathbf{R}=0$ is also a lattice point,

$$
\sum_{m} f_{m} e^{i \mathbf{G}_{\mathbf{m}} \cdot \mathbf{r}} e^{i \mathbf{G}_{\mathbf{m}} \cdot \mathbf{R}}=\sum_{m} f_{m} e^{i \mathbf{G}_{\mathbf{m}} \cdot \mathbf{r}} e^{i \mathbf{G}_{\mathbf{m}} \cdot 0}=\sum_{m} f_{m} e^{i \mathbf{G}_{\mathbf{m}} \cdot \mathbf{r}}
$$

$e^{i \mathbf{G}_{\mathbf{m}} \cdot R}=1$ must hold for all lattice points $\mathbf{R}$, i.e.

$$
\mathbf{G}_{\mathbf{m}} \cdot \mathbf{R}=2 \pi k
$$

where $k \in \mathbb{Z}$. The reciprocal lattice comprises all points $\mathbf{G}_{\mathbf{m}}$ which satisfy Eq. (3.9) for all lattice vectors $\mathbf{R}$. The reciprocal lattice is also a Bravais lattice; a convenient way to find the primitive reciprocal lattice vectors $\mathbf{b}_{\mathbf{i}}$ is through

$$
\left[\begin{array}{lll}
\mathbf{b}_{\mathbf{1}} & \mathbf{b}_{\mathbf{2}} & \mathbf{b}_{\mathbf{3}}
\end{array}\right]^{T}=2 \pi \mathbf{T}^{-1}
$$




\section{Miller indices}

Miller indices are a notation used to denote crystal planes and directions in Bravais lattices. Three integers $h, k$, and $l$ represent the family of planes orthogonal to the reciprocal lattice point $h \mathbf{b}_{\mathbf{1}}+k \mathbf{b}_{\mathbf{2}}+l \mathbf{b}_{\mathbf{3}}$ and are written as $(h k l)$. Negative integers are written with overbars, and Miller indices are typically written such that the greatest common divisor is $1 .\{h k l\}$ is used to denote the set of all planes equivalent to $(h k l)$ by symmetry. The distance $d_{h k l}$ between $(h k l)$ planes that intersect at least one lattice point is given by

$$
d_{h k l}=\frac{2 \pi}{\left\|h \mathbf{b}_{\mathbf{1}}+k \mathbf{b}_{\mathbf{2}}+l \mathbf{b}_{\mathbf{3}}\right\|}=\frac{2 \pi}{\left\|\mathbf{G}_{h k l}\right\|}
$$

Crystal directions are written with respect to the direct lattice rather than the reciprocal lattice; $[h k l]$ denotes the direction parallel to $h \mathbf{a}_{\mathbf{1}}+k \mathbf{a}_{\mathbf{2}}+l \mathbf{a}_{\mathbf{3}}$ and similarly $\langle h k l\rangle$ denotes the set of all directions symmetrically equivalent to $[h k l]$. For cubic crystal systems, $(h k l)$ is orthogonal to $[h k l]$, however this does not necessarily hold for other crystal systems.

A final convention used in diffraction is miller indices without any brackets, i.e. $h k l$; this is used to denote reflections or diffraction orders.

\section{Miller-Bravais indices}

For hexagonal systems such as AlN and GaN, two materials which crystallise in the wurtzite structure that are relevant within the context of this thesis, it is common to use a four-index notation $(h k i l)$ where $h, k, l$ are unchanged from $(h k l)$ and the extra index obeys $i=-(h+k)$. The advantage of this four-index Miller-Bravais index system is that it makes the 3 -fold symmetry apparent; e.g. $(1 \overline{1} 0) \equiv(1 \overline{1} 00)$ and $(010) \equiv\left(\begin{array}{lll}0 & 1 & 1\end{array}\right)$ are related by a $120^{\circ}$ rotation.

A direction $[h k l]$ can also be converted to Miller-Bravais notation 
$\left[\begin{array}{llll}H & K & I & L\end{array}\right]$ through the following relations:

$$
\begin{aligned}
H & =\frac{2 h-k}{3} \\
K & =\frac{2 k-h}{3} \\
I & =-\frac{h+k}{3} \\
L & =l
\end{aligned}
$$

Using this definition, the four-index direction $[h k i l]$ is orthogonal to the ( $h k i l$ ) plane, similar to the cubic crystal system.

\section{Laue equations}

The Laue equations describe the conditions required for an incident wave to be diffracted by a crystal lattice. Given an incident wavevector $\mathbf{k}_{\mathbf{i}}$ and a crystal lattice described by a transformation matrix $\mathbf{T}$ as above, Laue's condition for an outgoing wavevector $\mathbf{k}_{\mathbf{o}}$ can be written

$$
\mathbf{T}\left(\mathbf{k}_{\mathbf{o}}-\mathbf{k}_{\mathbf{i}}\right)=\mathbf{T} \Delta \mathbf{k}=2 \pi \mathbf{x}
$$

where $\mathbf{x} \in \mathbb{N}^{3} . \Delta \mathbf{k}=\mathbf{k}_{\mathbf{o}}-\mathbf{k}_{\mathbf{i}}$ is called the scattering vector. Multiplying Eq 3.13 on the left by $\mathbf{T}^{-1}$ gives

$$
\Delta \mathbf{k}=2 \pi \mathbf{T}^{-1} \mathbf{x}
$$

Noting that $2 \pi \mathbf{T}^{-1}$ is the definition of the reciprocal lattice from above, i.e. Laue's condition for diffraction is equivalent to saying that the scattering vector must be a point on the reciprocal lattice

$$
\Delta \mathrm{k}=\mathrm{G}
$$

\section{Elastic scattering}

Considering only elastic scattering of incoming waves, i.e. $\left\|\mathbf{k}_{\mathbf{i}}\right\|=\left\|\mathbf{k}_{\mathbf{o}}\right\|$, we can write

$$
\mathbf{G}+\mathbf{k}_{\mathbf{i}}=\mathbf{k}_{\mathbf{o}}
$$


Squaring both sides,

$$
\begin{aligned}
\|\mathbf{G}\|^{2}+2 \mathbf{G} \cdot \mathbf{k}_{\mathbf{i}}\left\|\mathbf{k}_{\mathbf{i}}\right\|^{2} & =\left\|\mathbf{k}_{\mathbf{o}}\right\|^{2} \\
\|\mathbf{G}\|^{2}+2 \mathbf{G} \cdot \mathbf{k}_{\mathbf{i}} & =0
\end{aligned}
$$

Using the fact that $-\mathbf{G}$ is also a reciprocal lattice point and combining with Eq. 3.11,

$$
\frac{2 \pi}{d}=2\left\|\mathbf{k}_{\mathbf{i}}\right\| \cos \phi
$$

where $\phi$ is the angle between $\mathbf{G}$ and $\mathbf{k}_{\mathbf{i}}$. Writing in terms of the wavelength $\lambda=2 \pi / k_{i}$ and using the complementary angle $\theta$ instead of $\phi$ results in Bragg's Law:

$$
\begin{aligned}
\frac{2 \pi}{d} & =2 \frac{2 \pi}{\lambda} \sin \theta \\
\lambda & =2 d \sin \theta
\end{aligned}
$$

\section{Diffracted Intensity}

While the above conditions for diffraction tell you what angles diffraction should be observed at, they say nothing about the intensity that should be observed. For a Bravais lattice with a given atomic basis, the intensity is related to the structure factor

$$
F(\mathbf{G})=A \sum_{i}^{n} f_{i}(s) e^{2 \pi i \mathbf{G} \cdot \mathbf{r}_{\mathbf{i}}}
$$

summing over the $n$ atoms in the basis. $A$ is a normalization factor, $f_{i}$ is the atomic scattering factor, which can be estimated for isolated atoms using e.g. the Cromer-Mann parameterization, dependent on the parameter $s$

$$
s=\frac{\sin \theta}{\lambda}=\frac{\|\mathbf{G}\|}{4 \pi}
$$

and $r_{i}$ is the location of the atom in the unit cell given in fractional coordinates. 


\section{Forbidden reflections}

Computing the structure factor for specific crystal systems reveals that certain reciprocal lattice vectors result in zero diffracted intensity. For example, the structure factor for a monatomic FCC crystal with atomic basis

$$
e=\left\{\left[\begin{array}{l}
0 \\
0 \\
0
\end{array}\right],\left[\begin{array}{c}
\frac{1}{2} \\
\frac{1}{2} \\
0
\end{array}\right],\left[\begin{array}{c}
\frac{1}{2} \\
0 \\
\frac{1}{2}
\end{array}\right],\left[\begin{array}{c}
0 \\
\frac{1}{2} \\
\frac{1}{2}
\end{array}\right]\right\}
$$

of a reciprocal lattice point $\mathbf{G}_{\mathbf{h k l}}$ is given by

$$
\begin{aligned}
F\left(\mathbf{G}_{\mathbf{h k l}}\right) & =A f(s)\left(e^{2 \pi i(0)}+e^{2 \pi i\left(\frac{h}{2}+\frac{k}{2}\right)}+e^{2 \pi i\left(\frac{h}{2}+\frac{l}{2}\right)}+e^{2 \pi i\left(\frac{k}{2}+\frac{l}{2}\right)}\right) \\
& =A f(s)\left(1+(-1)^{h+k}+(-1)^{h+l}+(-1)^{k+l}\right) \\
& = \begin{cases}4 A f(s) & h, k, l \text { all even or all odd } \\
0 & h, k, l \text { mixed parity }\end{cases}
\end{aligned}
$$

i.e. the reflections $111,200,220,311,222$ give a non-zero diffracted intensity while 100,210 etc. are all forbidden due to the mixed parity.

\subsubsection{Kinematic RHEED simulation}

Using the theory outlined above, it is possible to simulate RHEED patterns from arbitrarily defined crystal structures. This is implemented algorithmically following the program flowchart outlined in Fig. 3.1. A crystal structure is defined and the reciprocal lattice points $G_{h k l}$ and associated structure factors $f_{h k l}$ computed. Simulated RHEED patterns produced using this software can be directly compared to experimental observations, allowing viable structure models to be quickly identified. Additional details specific to the programmatic implementation such as the geometric setup and necessary simulation parameters and some limitations will be explained further below. 


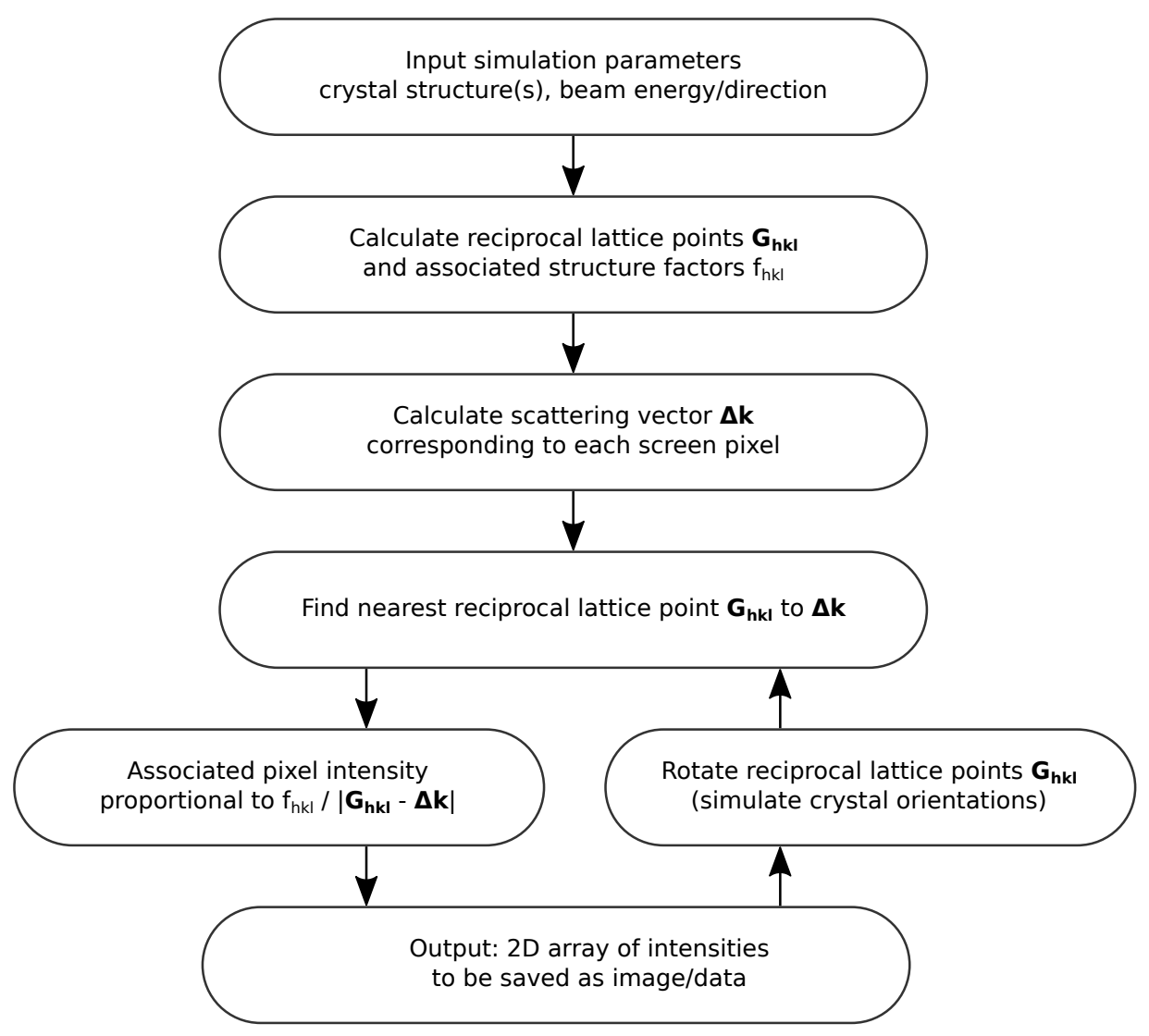

Figure 3.1: Program flowchart for the RHEED simulation code 


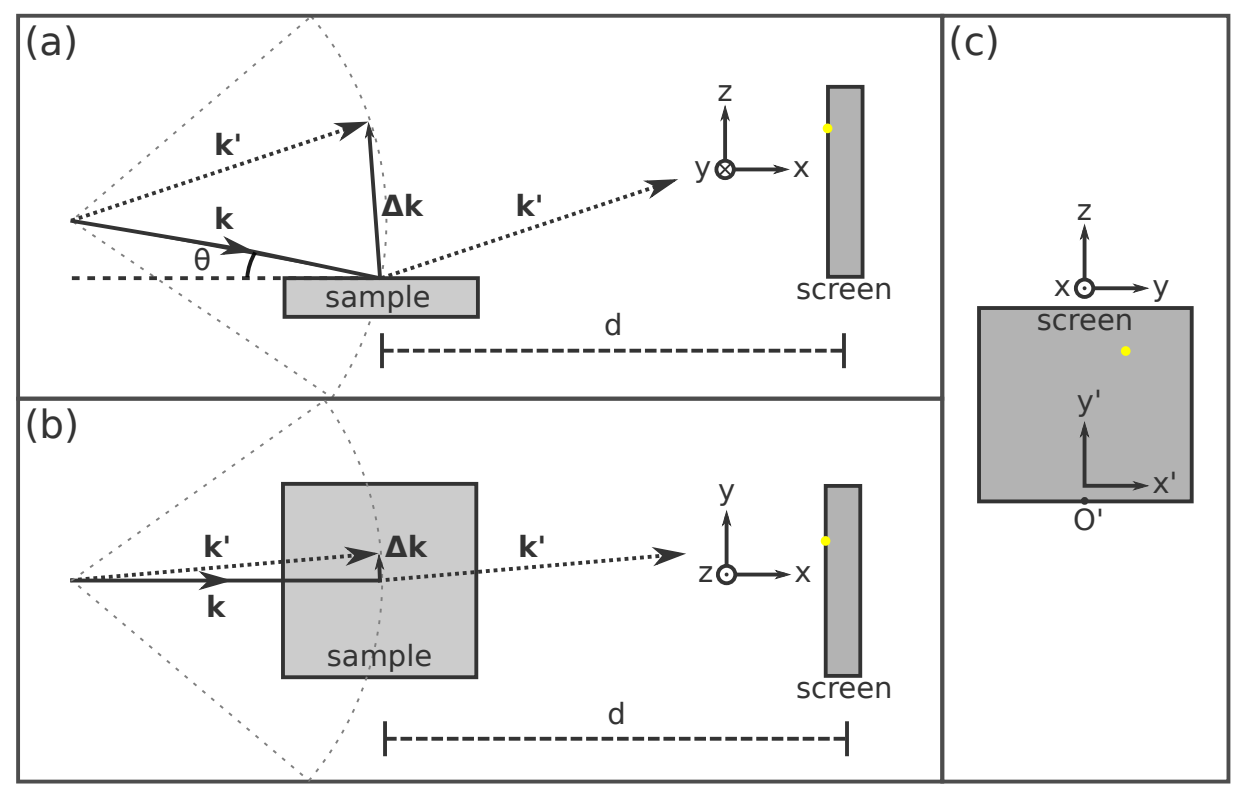

Figure 3.2: Schematic view of the RHEED simulation geometry from (a) side, (b) top and (c) screen view. The origin of both real and reciprocal space is defined as the point of incidence of the incoming electron beam, described by the wavevector $\mathbf{k}$, on the sample. The dashed arc marks the Ewald sphere. The yellow dot represents the incidence of a scattered wavevector $\mathbf{k}^{\prime}$ on the fluorescent screen. 


\section{Geometry}

The geometry of the simulation is shown schematically in Fig. 3.2, The coordinate system of the sample and the reciprocal lattice is denoted by $(x, y, z)$ with the origin $O$ set as the point where the electron beam $\mathbf{k}$ is incident on the sample. $d$ is the horizontal distance between the sample and the screen. The coordinate system of the screen is denoted by $\left(x^{\prime}, y^{\prime}\right)$ with its origin $O^{\prime}$ set at $(d, 0,0)$ giving a simple relationship between the two coordinate systems as

$$
\begin{aligned}
& x^{\prime}=y \\
& y^{\prime}=z
\end{aligned}
$$

The Laue diffraction condition can be understood geometrically as being satisfied where the Ewald sphere marked in Fig. 3.2 intersects with reciprocal lattice points; this is implemented algorithmically as explained below.

\section{Electron beam}

The incident electron beam is defined by two parameters, the electron energy $E$, in $\mathrm{keV}$, and the incident angle $\theta$, in radians. As displayed in Fig. 3.2, the electron beam is defined so that it has no $\mathbf{y}$ component, giving the incident electron beam wavevector $\mathbf{k}$ as

$$
\mathbf{k}=k_{0}\left(\begin{array}{c}
\cos \theta \\
0 \\
\sin \theta
\end{array}\right)
$$

where the magnitude of the incident electron beam wavevector can be calculated

$$
k_{0}=\frac{p}{\hbar}=\frac{1}{\hbar} \sqrt{\frac{2 m_{0} E}{c^{2}}+\frac{E^{2}}{c^{2}}}
$$

with $p$ the momentum of the electron, $m_{0}$ the mass of the electron and $c$ the speed of light. 


\section{Screen}

The screen is where the RHEED pattern is seen, resulting from the electron beam diffracting off the crystal sample. In practice, the screen is a window coated with a fluorescent phosphor which converts incident electrons into visible photons collected by a CCD camera. The size of the screen is defined by lower and upper bounds in the $x^{\prime}$ and $y^{\prime}$ directions. The distance from the sample to the screen $d$ is also specified. To compute the RHEED pattern algorithmically, the screen is divided into pixels. The chosen pixel width determins the final resolution of the simulation output, with a smaller width resulting in a higher resolution image at the expense of increased computation time.

\section{Calculation}

For a pixel located at $\mathbf{p}^{\prime}=\left(x^{\prime}, y^{\prime}\right)$ on the screen, the geometry defined in Section. 3.1 .2 gives the vector pointing from the origin to that pixel as $\mathbf{p}=$ $\left(d, x^{\prime}, y^{\prime}\right)$. For the kinematic approximation being used, we want to scale $\mathbf{p}$ so that it has the same magnitude as the incident wavevector; this gives us the diffracted wavevector $\mathbf{k}^{\prime}$ :

$$
\mathbf{k}^{\prime}=k_{0} \frac{\mathbf{p}}{\|\mathbf{p}\|}
$$

i.e. the wavevector leaving the sample that would hit the screen at point $\mathbf{p}^{\prime}$. The scattering vector $\boldsymbol{\Delta} \mathbf{k}=\mathbf{k}^{\prime}-\mathbf{k}$ is then used to calculate an intensity to associate with the pixel at $\mathbf{p}^{\prime}$.

A perfect infinite crystal produces a reciprocal lattice with point-like intensity and to satisfy the Laue diffraction conditions, the scattering vector $\Delta \mathbf{k}$ would have to match exactly with a reciprocal lattice point $G_{h k l}$. However for real, finite crystals with defects, the intensity is spread out over reciprocal space; this is done using a normalized 3D gaussian function. The intensity associated with a particular $\Delta \mathrm{k}$ is then

$$
I=I_{h k l} * \sum_{i} N_{i} \exp \left(\frac{-r_{i}^{2}}{\Gamma^{2}}\right)
$$


where $r_{i}$ is the proximity of $\boldsymbol{\Delta} \mathbf{k}$ to the $i$ th reciprocal lattice point in reciprocal space calculated using the standard Euclidean norm and $N_{i}$ is a normalization constant given by

$$
N_{i}=\frac{1}{\Gamma^{3}(2 \pi)^{\frac{3}{2}}}
$$

and $\Gamma$ a broadening factor that controls how much the intensity associated with a reciprocal lattice point is spread out over reciprocal space.

The negative exponential allows one to only consider the reciprocal lattice points closest to $\Delta \mathbf{k}$ using an appropriate heuristic to reduce the number of calculations.

This calculation is carried out for each pixel on the screen to produce the final RHEED pattern output.

\subsubsection{RHEEDSim GUI}

The parameters for a kinematic RHEED simulation are input using a graphical user interface (GUI) which can also display the output of a simulation. An overview of the GUI is shown in Fig. 3.3. The GUI is divided into sections; one window for editing the crystal lattices and orientations to be included in the simulation and another for specifying the remaining simulation parameters. The source code for a programmatic implementation of the process outlined above is freely available online[41]. A detailed description of the interface is presented below.

1. The Lattice Selection section allows multiple lattices to be included in a simulation, for example when considering a twinned system. Each lattice is selected edited and manipulated separately. The dimensionality of the selected lattice is also specified here, allowing a full 3D lattice to be defined (resulting in transmission spots) or a 2D lattice (resulting in diffraction streaks).

2. The lattice parameters for the selected lattice are specified in this section using the parametrization defined in 3.1.1. 


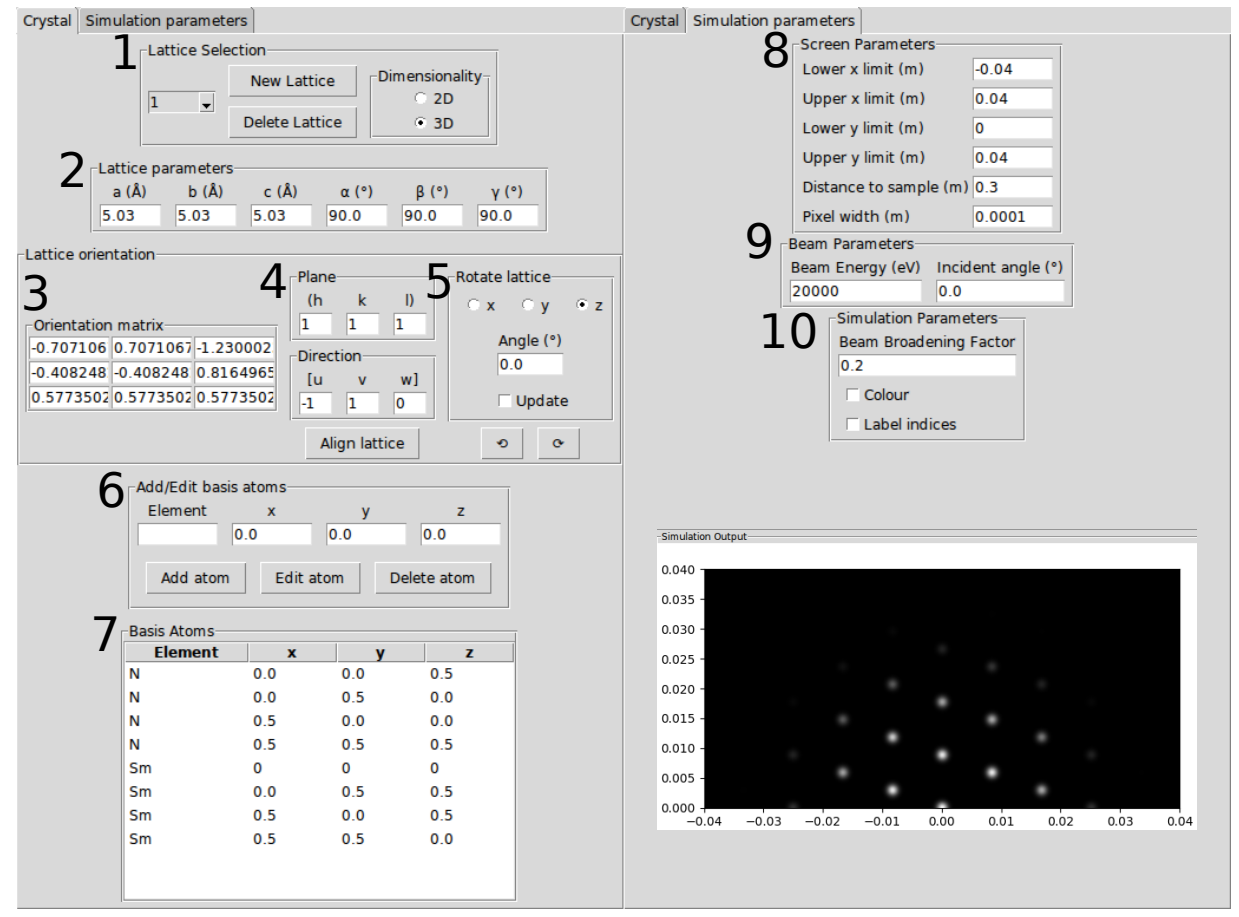

Figure 3.3: Graphical user interface for the RHEEDSim program. Sample parameters for a 3D diffraction pattern through a SmN crystal with the beam direction along the (111) [ 110$]$ azimuth with the output overlaid on the bottom right. 
3. The orientation of the lattice is saved in a $3 \times 3$ matrix (reduced to $2 \times 2$ for a $2 \mathrm{D}$ matrix), and is manipulated using the following operations

4. The orientation can be adjusted by specifying a lattice plane and a direction along that plane for the electron beam to be incident along, with the new orientation calculated when the "Align lattice" button is pressed. The Weiss Zone law must be satisfied for the resulting orientation to be meaningful; i.e. $h u+k v+l w=0$.

5. Further adjustments to the orientation of a lattice can be produced by rotating the lattice by a specified angle clockwise or anticlockwise around any of the $x, y$, or $z$ axes of the lab frame. The "update" option allows the simulation output to be automatically updated after a rotation is performed.

6. The atomic basis for a crystal lattice is input in this section by specifying an elemental symbol along with the fractional coordinates for the atom to be located at 3.1.1

7. A list of all the basis atoms that have been added to a lattice is stored and shown here, allowing atoms to be selected to edit or delete.

8. The screen parameters for the simulation are specified in this section according to the simulation geometry in 3.1 .2 with some typical values for a real system chosen as an example. The pixel width specified here determines the final resolution of the simulation output, with higher resolution images resulting from smaller pixel widths at the expense of longer computation time.

9. The beam parameters, namely the beam energy in $\mathrm{eV}$ and incident angle in degrees are specified here.

10. Some final miscellaneous simulation parameters are specified here. The beam broadening factor can be adjusted resulting in broader diffraction spots. The colour option allows the contributions from up to three 
lattices to be split into red, green and blue components respectively, resulting in an RGB colour image output instead of a grayscale pattern. The label indices option allows diffraction maxima to be labelled with the corresponding reciprocal lattice index.

\subsubsection{Summary}

A python program was written to simulate kinematic RHEED diffraction patterns from predefined crystal lattices. The code is flexible, allowing the simulation of arbitrarily oriented single crystal lattices or multiple crystal lattices, i.e. for crystal twinning. The graphical user interface allows one to easily modify parameters of a simulation, such as editing lattice structures or their orientations and quickly see the resulting changes to the output diffraction patterns.

\section{Discussion and limitations}

The beam broadening factor corresponding to $\Gamma$ in Eq. 3.30 is not derived from first principles; in essense it encompasses a number of limitations in the real experimental RHEED setup such as imperfections in the electron beam (finite beam size, non-monochromaticity) and crystalline material such as defects wich act to broaden reciprocal lattice points in reciprocal space, but the value used in the simulation parameter is not quantitatively obtained from these physical variables and instead is chosen to reproduce similar patterns to those obtained experimentally.

The kinematic approximation used in this simulation is limited in that it does not reproduce observed intensities accurately enough for some applications. Instead, more detailed analysis requires consideration of dynamic scattering events such as inelastic scattering and multiple scattering events. 


\subsection{Scanning probe microscopy}

Complementary to RHEED and other crystallographic techniques, Atomic force microscopy (AFM) and Scanning tunneling microscopy (STM) were used to study the surface morphology of thin films. Both techniques are forms of scanning probe microscopy which use a probe to scan the surface of a sample, employing different techniques to measure and control the distance between the sample and probe. Piezoelectric materials are used to control the motion of the scanning tip with the very fine precision required for the atomic resolution that scanning probe microscopy techniques are capable of. These techniques directly image the material surface, probing real-space, and complement the crystallographic techniques that rely on diffraction. The latter techniques instead probe reciprocal space, and provide information about the crystallographic order and periodicidy of the material.

\section{Atomic force microscopy}

Atomic force microscopy uses a sharp tip on the end of a cantilever to scan along the sample surface. A laser is directed onto the cantilever and reflected into a photodiode; as the tip moves across the sample and interacts with the surface, the reflection is also displaced, allowing the surface morphology to be recorded.

\section{Scanning tunneling microscopy}

Scanning tunneling microscopy uses a conducting tip to probe the surface of a conducting sample. A bias is applied between the sample and tip allowing electrons to tunnel between them. Measuring this tunneling current as a function of position on the sample is one way to record the surface morphology 


\subsection{X-ray diffraction}

X-ray diffraction is a ubiquitous tool for the characterization of atomic structures of materials, mainly crystalline. X-ray tubes are a common laboratory source for generating X-rays; a metal anode (e.g. copper) is supported inside a vacuum and bombarded with electrons accelerated through a high voltage, typically tens of kilovolts. A spectrum of x-ray photon energies characteristic of the anode material is emitted, and the highest intensity of emitted x-rays usually comes from the K-alpha transition; copper, for example, results in an x-ray wavelength of $\lambda=1.540598 \AA$. Synchrotron radiation is also used as an alternative high resolution, high intensity source of x-rays for diffraction.

The background theory outlined in 3.1.1 also applies to the elastic scattering of x-rays, although x-rays interact with the spatial distribution of electrons in a material compared to the electrons used in RHEED which interact strongly with matter through Coulomb forces. X-ray diffraction is applied using a number of different scattering geometries to probe the reciprocal lattice of crystalline materials to extract information complementary to that obtained using RHEED.

\subsubsection{X-ray optics}

A wide range of x-ray optics are used to control the properties of the incident and detected x-ray beam. Because the index of refraction of x-rays for most materials is less than one, manipulation cannot be done with lenses as is done with visible light and we are restricted to reflection and diffraction methods. With regards to powder and thin film XRD, there are two focusing methods normally employed; a pseudo-parafocusing geometry has a divergent beam incident on a curved sample which diffracts completely at the detector point. Alternatively, the incident beam is focused into a parallel beam while the detector is also fitted with collimators to ensure the detector is picking up only x-rays being diffracted at the correct angles. The parallel beam geometry offers some advantages over the more typical parafocusing 
geometry, however it requires the use of more complicated optics.

\section{Parallel beam}

It can be preferrable to employ a parallel beam geometry rather than the typical parafocusing geometry with a diverging beam. Using a parallel beam can reduce or even eliminate many errors due to sample alignment errors which thin film samples are prone to. A parabolically curved multilayer structure called a Göbel mirror is used to reflect the diverging x-ray source into a parallel beam with very low divergence while also helping to monochromatize the beam. It is also possible to combine additional monochromators made from single crystals of Si or Ge to further improve the quality of the beam at the expense of intensity. This is preferrable for certain x-ray measurements such as grazing incidence XRD (GIXRD) and high resolution XRD (HRXRD). It is also essential for a non-diffraction technique, x-ray reflectivity (XRR), covered further in Section 3.4 .

\subsubsection{Geometry}

In the most flexible set-up, the orientation of crystalline samples is controlled with a four circle goniometer system. The sample is placed at the center of the goniometer and can typically be rotated in two perpendicular axes, $\phi$ and $\psi$, while the angles of the incident beam and detector are also independently controllable through the $\omega$ and $2 \theta$ axes respectively. The linear position of the sample is also finely tuneable in the $x, y, z$ directions. As the x-ray wavelength is typically fixed, different measurements are made by controlling the sample orientation and the angles of the detectors which, in effect, maps out different sections of reciprocal space to provide information about the crystallinity of the sample. 


\section{Bragg-Brentano}

The Bragg-Brentano geometry is widely used for powder diffraction experiments. The scattering angle is restricted to perpendicular to the sample by adjusting the $2 \theta$ angle such that it is always double the incident angle $\omega$; it is sometimes also called a $2 \theta-\theta$ scan (or $\theta-2 \theta$ when plotted with respect to the incident angle). Only information about the planes perpendicular to the sample geometry is obtained, but this is usually more than enough for properly prepared powder samples which should have randomly oriented crystallites in all directions to diffract at all the expected planes. For thin film samples which can be textured, the Bragg-Brentano geometry is less than ideal; it still gives some information about the crystal phases in the sample but is restricted by the small illuminated volume from the thin film and a strong signal from single-crystal substrates which can sometimes outshadow the film's signal.

\section{Asymmetric scans}

A lot of information can be gleamed from asymmetric scans compared to the symmetric scan geometry used in the Bragg-Brentano setup. The most general form is encompassed in reciprocal space mapping, where an entire region of reciprocal space can be mapped iteratively by continuously varying the sample orientation and incident and detector angles. More specific scans map out slices of reciprocal space, such as rocking curves where the incident and detector are fixed on a known reflection and the sample is tilted, i.e. scanning over the $\omega$ axis while keeping $2 \theta$ fixed, or $\phi$-scans which instead rotate the sample, allowing the rotational symmetry of the crystal to be recorded. 


\subsection{X-ray Reflectivity}

X-ray reflectivity is a technique used to characterise thin film structures. A monochromatic beam of x-rays is incident on a sample and the specularly reflected intensity is measured as a function of incidence angle. This reflected intensity depends on the electron density profile of the sample being measured; in practice, this allows extraction of properties of the sample such as layer thicknesses, layer densities and surface and interface roughnesses. Note that this does not depend on the crystallinity of the material and works equally well on amorphous layers as well as epitaxial or polycrystalline layers. A model density profile is combined with the aformentioned sample parameters and used to simulate a theoretical reflectivity curve. The model parameters are refined using a genetic algorithm optimization process until the calculated theoretical curve matches the experimentally obtained data. The software used for this simulation and parameter fitting is PANanalytical X'Pert Reflectivity. Example simulations of a single layer on top of a substrate will be presented later to show how each sample parameter affects the output reflectivity profile.

\subsubsection{XRR at VUW}

The ability to perform basic XRR measurements at VUW was developed during this project. The PANalytical XRD machine located there is primarily used for powder XRD measurements, with the basic optics required for the parallel beam geometry present but otherwise unused. A Göbel mirror is used to reflect the $\mathrm{x}$-rays exiting the $\mathrm{x}$-ray tube into a monochromatic, parallel beam with a low divergence. A parallel plate collimator is used on the detector side to ensure the detected x-rays are also parallel with being diffracted or reflected from the sample. While this setup is capable of basic XRR measurements and other measurement techniques that take advantage of a parallel beam setup, it lacks the flexibility of a 3- or 4-circle goniometer which would allow motorized movement of a sample for alignment purposes. 


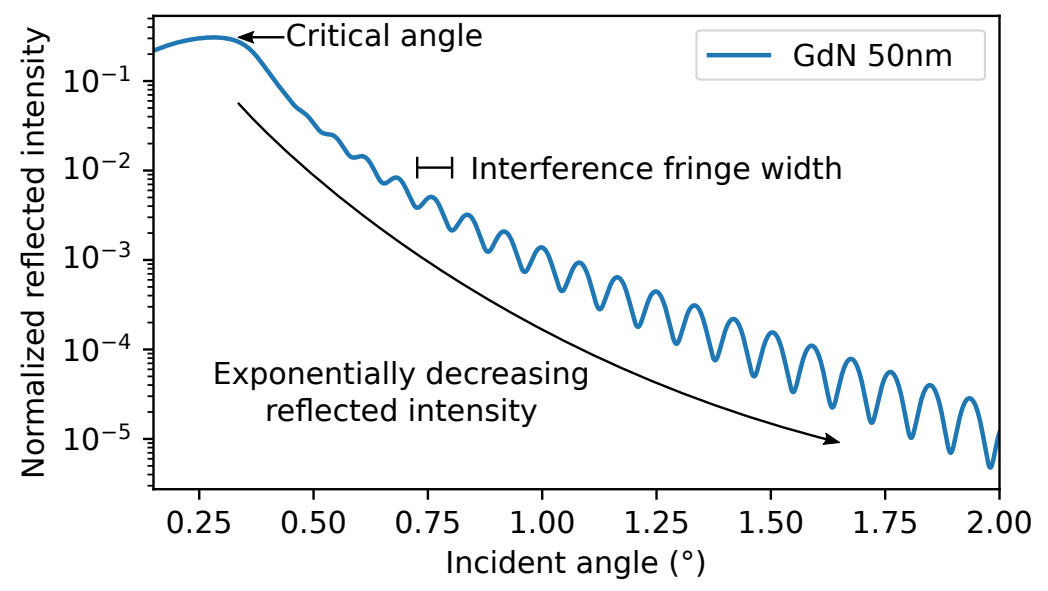

Figure 3.4: Example simulated reflectivity profile from a single GdN layer on a $\mathrm{SiO}_{2}$ substrate.

Correct alignment is essential for high quality XRR measurements to more accurately extract most of the sample parameters.

Additionally, while the Göbel mirror acts as a partial monochromator, it does not completely separate the $k \alpha_{1}$ and $k \alpha_{2}$ components of the copper $\mathrm{x}$-rays and the outgoing beam divergence may not be low enough for some applications. Single crystal monochromators made from Si or Ge are used for this purpose to give even lower divergence with higher monochromaticity. This could possibly allow for even thicker films to be analysed with XRR compared to the experimental limit with just the Göbel mirror of around $150 \mathrm{~nm}$.

\subsubsection{Example reflectivity profiles (simulated)}

A simulated reflectivity profile of a single GdN layer on a $\mathrm{SiO}_{2}$ substrate is shown as an example in Fig. 3.4. Some key features of general reflectivity profiles are indicated. The critical angle is the angle below which total external reflection of incident x-rays occurs. Interference fringes are observed due to the (electron) density difference between the GdN layer and the sub- 


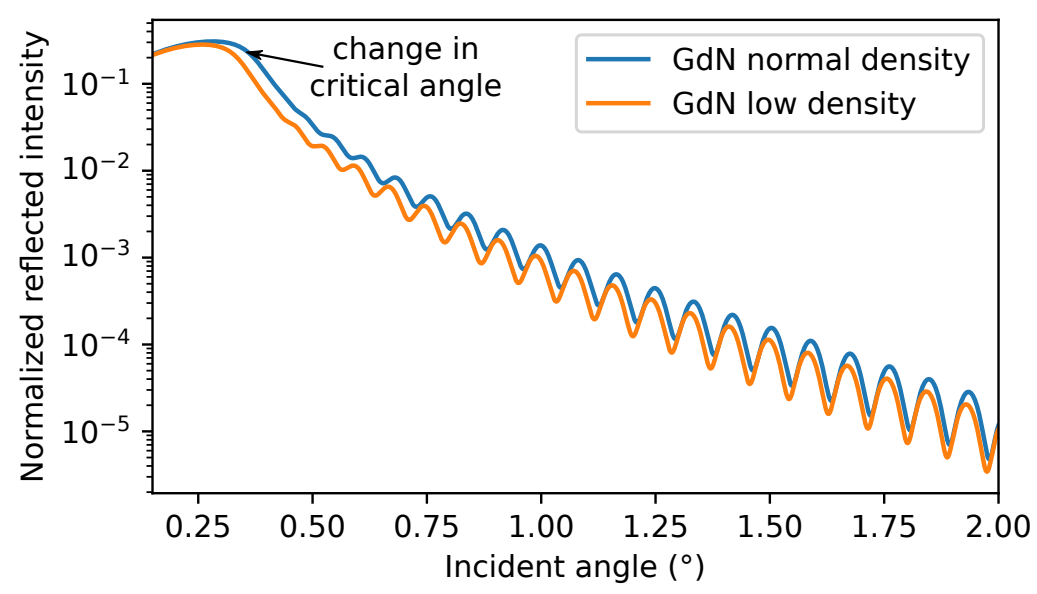

Figure 3.5: Example reflectivity profiles from single layers with different densities, showing the influence on the critical angle.

strate and the sharp interface between them. The reflectivity is seen to decrease exponentially with increasing incident angle; in practice, this limits the amount of useful information that can be obtained before noise outweighs the reflectivity signal.

\section{Density}

Fig. 3.5 shows the effect of changing the density of the top layer, with a lower density causing the critical angle to shift to a lower value. This is due to the effect of density on the refractive index of the material changing the angles at which total external reflection of x-rays can occur. The density between different materials can differ significantly meaning XRR can act as preliminary material identification tool.

\section{Layer thickness}

Fig. 3.6 shows the effect of having layers of different thicknesses. The $100 \mathrm{~nm}$ thick layer has much smaller interference fringe widths than the $50 \mathrm{~nm}$; as 


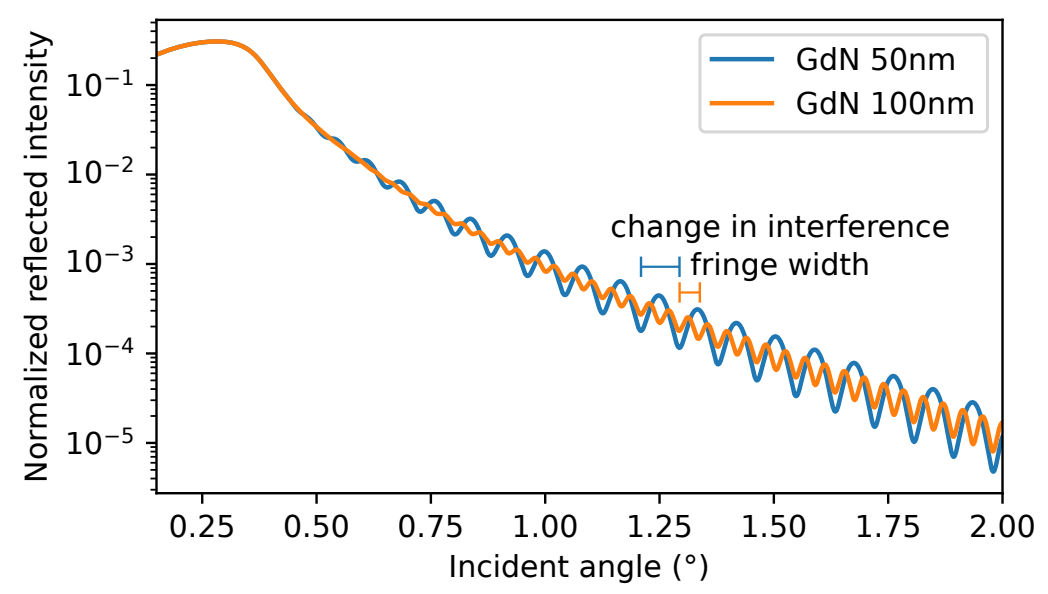

Figure 3.6: Example reflectivity profiles from single layers with different layer thicknesses, showing the influence on interference fringe width.

such, higher scan resolution is more important when trying to measure thicker films. Much thinner films on the order of a few $\mathrm{nm}$ instead require more intensity to see fringes that appear at higher incident angles.

\section{Surface and interface roughness}

Fig. 3.7 shows the effect of increasing the surface and interface roughnesses in a sample. The interface roughness, that is, the roughness between the GdN layer and the substrate acts to dampen the size of the interference fringe in terms of the observed intensity difference between the peaks and troughs. If the interface roughness is too high, it may become impossible to observe any interference fringes at all. On the other hand, surface roughness increases the rate of exponential decay of the reflectivity with incident angle, which in practise limits the number of fringes you can observe before noise outweighs the signal. These effects limit the type of samples that are suitiable for XRR measurements to relatively smooth films. 


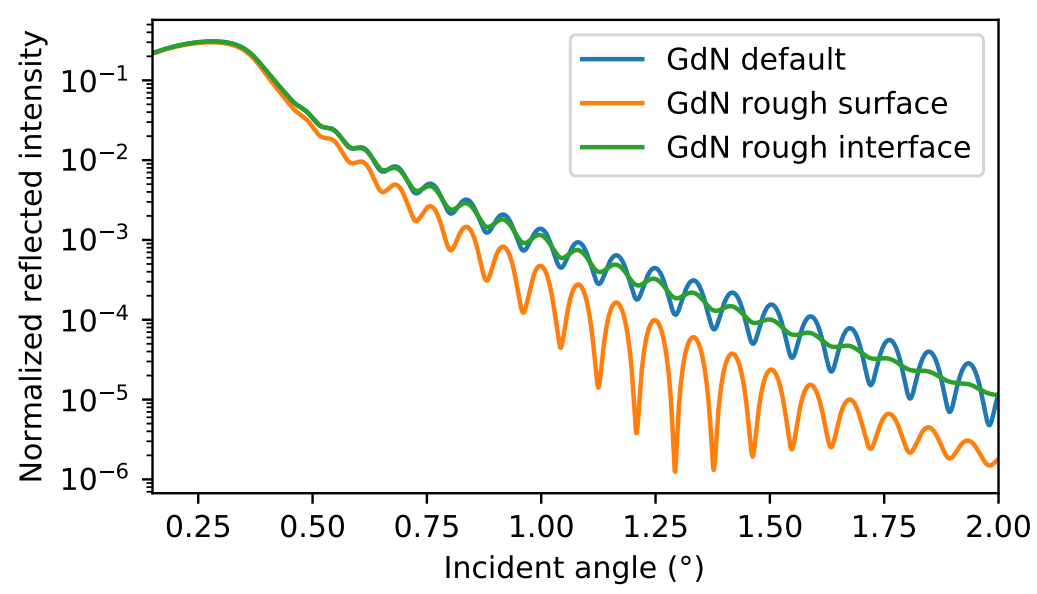

Figure 3.7: Example reflectivity profiles from single layers with increased surface and interface roughnesses respectively, changing the intensity variation observed in the interference fringes.

\subsubsection{Multilayer systems}

While parameters are relatively easy to extract from reflectivity of single layer systems as shown above, the presence of multiple layers necessitates curve fitting with sample models using software due to the complex interference that occurs between the multiple layers. In this situation, a good initial guess for the true sample model is needed to avoid finding local minima giving incorrect layer thicknesses.

\subsection{Rutherford Backscattering Spectrometry}

Rutherford Backscattering Spectrometry (RBS) is an analytical technique used to study the composition of thin films. High energy ions $(\sim 2 \mathrm{MeV})$ of a light element such as $\mathrm{He}^{+}$are fired at a target sample and the energy distribution of elasically backscattered ions is detected. Using conservation of momemtum and kinetic energy for the collision, the energy $E_{1}$ of the scattered 
particle is reduced from the initial energy $E_{0}$ by the following relation

$$
\frac{E_{1}}{E_{0}}=k=\left(\frac{m_{1} \cos \theta_{1} \pm \sqrt{m_{2}^{2}-m_{1}^{2}\left(\sin \theta_{1}\right)^{2}}}{m_{1}+m_{2}}\right)
$$

$k$ is called the kinematical factor. $m_{1}, m_{2}$ are the masses of the scattering ion and the target nucleus respectively and $\theta_{1}$ the scattering angle in the lab frame of reference. Additionally, the incident ions also lose energy through small angle scattering from the electron distribution in the sample. This energy loss is expressed in terms of the material-dependent stopping power $S:$

$$
S(E)=\frac{d E}{d x}
$$

Simulation software combines these basic principles and more to compute energy spectra from thin film models with given parameters to reproduce experimentally obtained data, allowing the determination of compositional depth profiles.

\subsection{Cross-section Scanning Electron Mi- croscopy}

Cross-section scanning electron microscopy (SEM) is a technique used to measure the thicknesses of deposited layers. Electrons emitted from a hot filament are focussed using apertures and electromagnetic lenses onto a sample to be imaged. The surface is scanned using deflections coils to direct the beam across the sample surface. Secondary electron imaging is primarily used for the imaging in this thesis; secondary electrons are emitted from near the surface of the sample due to inelastic interactions between the electron beam and the sample. These secondary electrons have lower energy than elastically backscattered electrons and are useful for investigating sample surface topography.

To prepare the thin film samples for cross-section SEM, they are cleaved using a diamond pen to expose a fresh cross-section of the layer stack and 


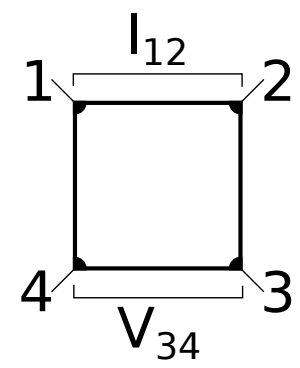

Figure 3.8: van der Pauw geometry used for four-terminal electrical measurements on thin film samples. Four contacts are placed on the edge of a homogeneous sample with no holes and a current is passed through one pair of contacts while the voltage between the other pair is recorded.

then mounted onto sample holders and introduced into the SEM without any coating. The samples are then aligned at the desired beam current and voltage to produce adequate contrast for layer thickness extraction. A ZEISS Supra 40 was used for the SEM measurements presented in this thesis.

\subsection{Other Characterisation Methods}

Several other standard measurement techniques were used to analyse REN thin films presented in this thesis to characterise the electrical and magnetic properties. To study the properties of these films at low temperatures, several separate specially designed liquid helium-cooled cryostats are required to allow measurements down to $4 \mathrm{~K}$

\subsubsection{Electrical characterisation}

Electrical characterisation such as the temperature dependent resistivity or carrier concentration of thin films is generally performed using a four terminal measurement using the van der Pauw geometry, which can be applied to twodimensional samples of arbitrary shape (Fig. 3.8). Four ohmic contacts are 
placed on the perimeter of a sample, labelled 1 through 4 in clockwise order. A measurement is made by sending a current through one pair of contacts, e.g. $I_{12}$ for a positive current injected in contact 1 through contact 2 , and the induced voltage is measured across the remaining contacts, e.g. $V_{34}$. A corresponding resistance value is calculated:

$$
R_{12,34}=\frac{V_{34}}{I_{12}}
$$

This measurement is repeated for all six possible current/voltage contact pairs; diagonal pairs measure the hall effect when a magnetic field is applied normal to the sample. The average sheet resistance, $R_{S}$ has units of $\Omega / \square$ and is related to the resistances measured by the van der Pauw formula

$$
e^{-\pi R_{12,34} / R_{S}}+e^{-\pi R_{23,41} / R_{S}}=1
$$

Additional accuracy can be obtained by using reciprocal and reversed polarity measurements; define

$$
\begin{aligned}
R_{\text {vertical }} & =\frac{R_{12,34}+R_{34,12}+R_{21,43}+R_{43,21}}{4} \\
R_{\text {horizontal }} & =\frac{R_{23,41}+R_{41,23}+R_{32,14}+R_{14,32}}{4}
\end{aligned}
$$

The van der Pauw formula then takes the form

$$
e^{-\pi R_{\text {vertical }} / R_{S}}+e^{-\pi R_{\text {horizontal }} / R_{S}}=1
$$

There is no general solution for $R_{S}$; for the special case $R_{\text {vertical }}=R=$ $R_{\text {horizontal }}, R_{S}$ is given by

$$
R_{S}=\frac{\pi R}{\ln 2}
$$

Otherwise, a numerical method such as Newton-Raphson can be used to solve for $R_{S}$ recursively. The bulk resistivity of a uniform thin film sample is given by

$$
\rho=R_{S} \cdot T
$$

where $T$ is the thickness of the film. 


\section{Hall measurement}

The van der Pauw geometry also allows for the Hall effect to be measured when a magnetic field $B$ is applied perpendicular to the sample. If we consider a material with only one carrier type, e.g. electrons, the Hall voltage $V_{H}$ measured perpendicular to the direction of current $I$ in the steady state is given by

$$
V_{H}=\frac{I B}{q n t}
$$

where $q$ is the elementary charge and $n$ is the carrier density. It is common to define the hall coefficient $R_{H}$ as

$$
R_{H}=\frac{V_{H} t}{I B}=\frac{1}{n q}
$$

We can also define a Hall resistivity $\rho_{H}$

$$
\rho_{H}=\frac{V_{H}}{I} t
$$

\section{Anomalous Hall effect}

In ferromagnetic materials, there is an additional contribution to the Hall resistivity due to scattering resulting from spin-orbit coupling proportional to the perpendicular magnetization in the sample [42], empirically described by

$$
\rho_{H}=R_{H} B+\mu_{0} R_{S} M_{\perp}
$$

To extract the carrier concentration from Hall measurements in this regime, it is thus necessary to take the measurements at high field where the sample magnetization is saturated and the anomalous Hall effect no longer contributes to the recorded signal.

\section{Measurement apparatus}

A helium closed-cycle cryostat is used to perform temperature-dependent resistivity measurements down to liquid helium temperatures around $4 \mathrm{~K}$. 
The operation of this cryostat is more fully described elsewhere 43. A commercial Quantum Design Physical Property Measurement System (PPMS) is used to measure electric transport properties using a rotating sample stage in magnetic fields of up to $8 \mathrm{~T}$ and down to as low as $2 \mathrm{~K}$. For both the closed-cycle cryostat and PPMS, measurement sequences are defined, including details such as temperature ranges, temperature ramp rates and electric currents and magnetic field strengths to be applied to the sample, and data is recorded to the computer.

\subsubsection{Magnetic characterisation}

The magnetic properties of thin film samples are measured using laboratory magnetometry techniques, such as Superconducting quantum interference device (SQUID) or Vibrating Sample Magnetometry (VSM). Examples of measurements employed are zero-field cooled/field-cooled (ZFC-FC) curves, where the magnetic moment is measured while the sample is cooled down in zero field and heated up in varying applied field strengths. Alternatively, one measures the magnetic moment as a function of applied field at fixed temperatures. The resulting curve is called a hysteresis loop.

\section{SQUID}

SQUID magnetometry is based on the Josephson effect; two Josephson junctions are connected in parallel in a superconducting loop, and it is possible to measure changes in the magnetic flux threading this loop as oscillations in voltage across the junction corresponding to the magnetic flux quantum.

It is necessary to know the total volume of the thin film itself (i.e. the film thickness) to extract the magnetic properties per unit mass or volume of the material being studied. The surface area of a sample to be measured in the SQUID (limited to about $\left.(5 \mathrm{~mm})^{2}\right)$ can be calculated by measuring the mass of the sample and using the known density and thickness of the substrate being used. The sample must also be carefully mounted while taking care 
not to introduce magnetic contamination which can easily overshadow the film material. The magnetic contribution from the substrate itself must also be accounted for and removed. This is done most accurately by measuring a sample of the substrate alone using the same sequence to be performed on the thin film sample.

A commercial Quantum Design Magnetic Property Measurement System (MPMS) is used to measure the sample magnetic moment down to $2 \mathrm{~K}$ and in fields of up to $7 \mathrm{~T}$. Measurement sequences are defined to perform the temperature dependent ZFC-FC and field-dependent hysteresis measurements described above. The MPMS is primarily operated using the reciprocating sample option, where the sample is moved up and down through a second order gradiometer detection coil coupled to the SQUID detector. The magnetization of the sample is extracted from the sample position-dependent SQUID voltage measurements.

\section{Curie-Weiss law}

The temperature dependence of the magnetic susceptibility $\chi$ of a ferromagnetic material above its magnetic ordering temperature in the paramagnetic regime is empirically described by the Curie-Weiss law:

$$
\chi=\frac{M}{H}=\frac{C}{T-\theta_{C}}
$$

where $M$ is the sample magnetization, $H$ the externally applied magnetic field, $C$ the material-specific Curie Constant, $T$ the temperature of the material and $\theta_{C}$ the Curie-Weiss temperature. The Curie constant is equal to

$$
C=\frac{\mu_{0} \mu_{B}^{2}}{3 k_{B}} N g^{2} J(J+1)
$$

where $\mu_{0}$ is the permeability of free space, $\mu_{B}$ the Bohr magneton, $k_{B}$ the Boltzmann constant, $N$ the number of magnetic atoms or ions, $g$ the Landé gfactor and $J$ the angular momentum quantum number. An effective magnetic moment $\mu_{\text {eff }}$ can be defined as

$$
\mu_{e f f}=\mu_{B} g \sqrt{J(J+1)}
$$


For the case of $\mathrm{Gd}^{3+}, g=2$ and $J=\frac{7}{2}$ giving an effective moment of $\mu_{\text {eff }}=$ $7.937 \mu_{B}$. It is convenient to plot the inverse susceptibility vs temperature:

$$
\frac{1}{\chi}=\frac{T-\theta_{C}}{\frac{\mu_{0} N}{3 k_{B}} \mu_{e f f}^{2}}
$$

This relation is used to estimate the Curie temperature $T_{C}$ in temperature dependent magnetic measurements of $\operatorname{GdN}\left(T_{C} \approx \theta_{C}\right.$ for a first-order ferromagnetic transition, hereafter used interchangeably) with a linear fit to the paramagnetic region $\left(T \gg T_{C}\right)$. Additionally, the number of magnetic ions $N$ corresponding to the ferromagnetic transition at $T_{C}$ is extracted and compared to the calculated number of Gd ions in a measured sample. 


\section{Chapter 4}

\section{Study of the preferential growth of GdN}

\subsection{Introduction}

Gadolinium nitride is the most studied of the rare earth nitride series. It crystallises in the rock salt structure with a lattice parameter of about $4.98 \AA$. It is widely accepted that GdN is both intrinsically ferromagnetic and semiconducting 23], with n-type conductivity usually observed due to doping by nitrogen vacancies $\left(V_{N}\right)$ which donate as many as three electrons per $V_{N}$. GdN has the highest ferromagnetic transition temperature among the RENs, often reported to be between $50 \mathrm{~K}$ to $70 \mathrm{~K}[26,34$. It is notably a soft ferromagnet, with a large saturation magnetisation of up to $7 \mu_{B} / \mathrm{Gd}^{3+}$ observed at low temperatures in highly ordered epitaxial thin films and a coercivity on the order of 100 Oe [36]. The growth conditions for GdN thin films are known to affect both the electronic and magnetic properties, in particular the $V_{N}$ concentration is affected by the $\mathrm{N}_{2}$ : Gd ratio during deposition. High $\mathrm{N}_{2}$ : Gd ratios $(>100)$ have generally applied to minimise the $V_{N}$ concentration, and to avoid the formation of unwanted metallic clusters [19, 25]. In the case of epitaxial growth which requires high substrate temperatures above $500{ }^{\circ} \mathrm{C}$, the low $V_{N}$ formation energy of $\sim 0.5 \mathrm{eV}$ ensures a $V_{N}$ concentration on the 
order of $\sim 0.01$ regardless of the $\mathrm{N}_{2}$ :Gd ratio.

Many effects of the individually controllable deposition parameters during growth of GdN and other REN thin films are not fully understood. This chapter explores the room temperature growth of polycrystalline GdN and in particular how growth parameters such as the GdN film thickness and the respective $\mathrm{Gd}$ and $\mathrm{N}_{2}$ fluxes affect the structural properties of the deposited films. The structural properties, primarily characterised by x-ray diffraction, are correlated to the resulting electronic transport and magnetic properties.

One key structural characterisation of deposited thin films that will be focused on is the crystallographic texture. A thin film is textured if the orientations of crystallites are not uniformly random and instead have preferred orientations. The formation of such textures is generally a function of the properties of the material to be deposited as well as the deposition conditions, as will be expanded on below. The preferential orientation of various rock salt structure materials has been long been studied, for example, an early study showing preferred orientations of (111) for $\mathrm{LiF}$ and (110) for $\mathrm{NaCl}$ [44], and more recently a kinetic model developed to explain and predict the preferred orientation of $\mathrm{MgO}$ grown on amorphous substrates [45].

For the RENs, it has been observed that many of then show a tendency to preferentially grow with a strong (111) orientation when deposited polycrystalline. This has been taken advantage of by different groups for the epitaxial growth of REN thin films. Instead of using a cubic lattice-matched substrate for which suitable choices are limited, GaN and AlN which crystallise in the hexagonal wurtzite structure can be used. The close-packed (111) plane of the REN is matched to the close-packed (0 001 ) plane of the wurtzite structure; heteroepitaxial growth of GdN and $\mathrm{SmN}$ on GaN and AlN with this relationship has been successfully demonstrated by different groups worldwide 24, 25, 28, 34, 37]. Understanding of the roots of this preferential orientation also lends itself to further understanding of the epitaxial growth mode on such surfaces.

The basic technique behind the growth of thin films of rare earth nitrides 
is deceptively simple; solid sources of high purity rare earth metals are heated to form a vapour which is allowed to deposit on a chosen substrate in the presence of nitrogen gas. However, despite the simplicity of this concept, there is much that is not completely understood about the growth process at the nanoscale. Of particular interest is the fact that no activation of the molecular $\mathrm{N}_{2}$ gas is required for the formation of the rare earth nitride. The strong triple bond in $\mathrm{N}_{2}$ is catalytically broken providing the atomic nitrogen for incorporation into the growth front of the film. The catalytic breaking of $\mathrm{N}_{2}$ is not new in itself; it has been studied for many transition metals and is used extensively in the Haber process for the fixation of nitrogen to ammonia using iron-based catalysts, for example. With regards to REN film growth, the catalysis adds another dimension to what needs to be understood for optimisation of the growth process via molecular beam epitaxy.

\subsection{Thin film growth and texture formation}

The formation of crystallographic texture in thin films has three principle contributing factors; preferentially oriented nucleation, evolutionary selection of preferred growth orientations, and post-deposition recrystallisation [46]. The evolutionary selection aspect is the factor that is primarily explored in this chapter; preferentially oriented nucleation is intentionally minimised by using amorphous substrates upon which nucleation should be random, and post-deposition recrystallisation is usually a result of annealing processes which are not explored here.

Evolutionary selection is understood to result from the fact that different crystal faces grow at different rates. This specifically means that the vertical growth rate of any given crystallite is orientation dependent. Given an initial deposited layer consisting of randomly oriented crystallites, there will be an evolutionary pressure selecting for those crystallites which are oriented with a fast growing axis perpendicular to the substrate surface. As film thickness increases, more and more of the misoriented crystallites will "die" as 


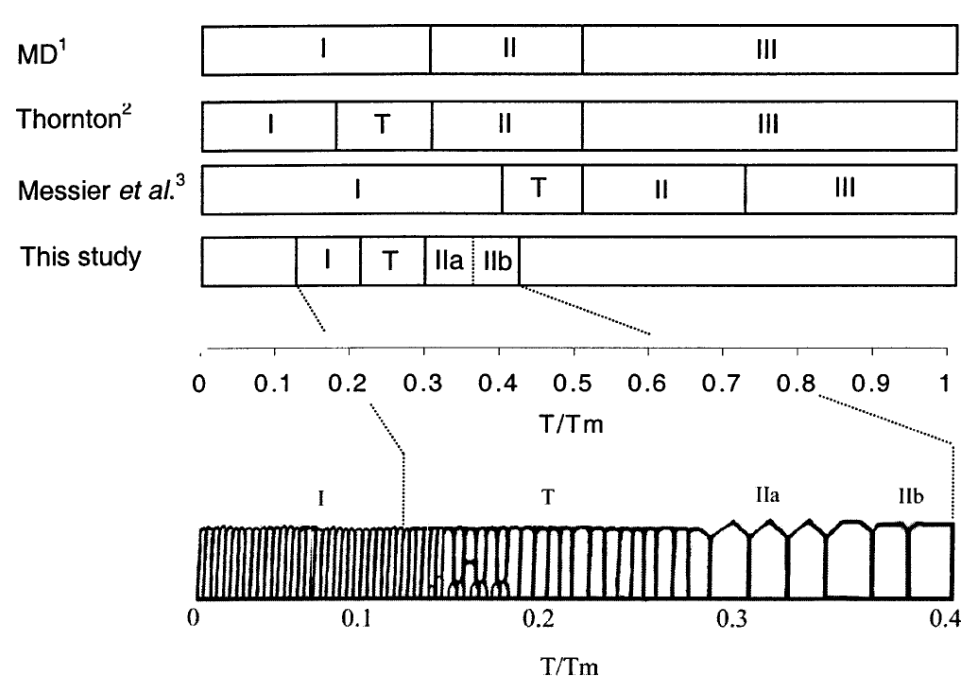

Figure 4.1: A comparison of various structure zone models relating the homologous temperature $T / T_{m}$ to the resulting microstructure of a deposited thin film, published by MD[47], Thornton[48], Messier et al. 49]. Reprinted with permission from [50]. (C) 2004 American Chemical Society.

the faster-growing crystallites begin to dominate. It is through this mechanism that texture is observed to develop even from a randomly oriented polycrystalline layer.

Regarding the growth of GdN and other RENs which are grown through the catalytic reaction of molecular $\mathrm{N}_{2}$ with rare earth adatoms on the substrate, any preferential orientation is of particular interest as it should reveal some information about how the reaction to incorporate incoming $\mathrm{N}_{2}$ into the film bulk takes place.

\subsection{Thin film microstructural evolution}

Although we are only looking at room temperature deposition, it is also important to understand how the substrate temperature generally affects the structure observed in thin film growth systems. Structure zone models have 
been developed to understand a wide number of material systems 47 51. A key parameter is the homologous temperature $T / T_{m}$, where $T$ is the substrate temperature during deposition and $T_{m}$ the melting point of the material being deposited. A number of zones have been identified generalising microstructures observed in a variety of thin film material systems. Fig. 4.1 taken from [50] shows how the homologous temperature results in different film microstructures due to the changes in adatom diffusion and grain coalescence. In the case of room temperature deposition of GdN and estimating $T_{m}$ to be $\sim 3200 \mathrm{~K}$ [52], $T / T_{m}$ is approximately $300 \mathrm{~K} / 3200 \mathrm{~K} \sim 0.095$ and falls well into Zone $\mathrm{I}\left(T / T_{m} \lesssim 0.3\right)$. This zone in other material systems is characterised by films with needle-like columnar or fibre growth with heavily voided boundaries due to the low adatom diffusion and shadowing effects. The columns are generally not single crystal but composed of equiaxed grains.

At higher temperatures, increased surface diffusion leads to columnar grains with larger grain width (Zone II). Zone III occurs at even higher temperatures at which bulk diffusion dominates, resulting in equiaxed grains of increasing size. Extensions to the structure zone model have been developed to include energetic particle bombardment, for example.

Aside from the deposition temperature, we are interested in understanding how the other controllable growth parameters (respective $\mathrm{Gd}$ and $\mathrm{N}_{2}$ fluxes and film thickness) influence the microstructure and other properties of the film. The catalytic nature of the growth of GdN from evaporating Gd in a low partial pressure of nitrogen adds additional aspects to the growth to be understood that are not included in the structure zone models noted above, which have been extensively studied for metallic systems.

\subsection{Sample growth and measurement}

Polycrystalline thin films of GdN of varying thicknesses were deposited on amorphous fused silica substrates at ambient temperature in an ultra high vacuum chamber with a base pressure of $<10^{-8}$ mbar. Amorphous substrates 
were chosen to minimise the effect of preferential nucleation that would result from a crystalline substrate, as mentioned in Section. 4.2. The substrates were outgassed at $280^{\circ} \mathrm{C}$ for 1 hour and left to cool for at least 2 hours before growth. $99.9 \%$ purity Gd metal was evaporated via electron bean evaporation in the presence of a nitrogen partial pressure in the range of $3 \times 10^{-5}$ to $6 \times 10^{-5}$ The GdN deposition time was varied between 6 minutes and two hours to produce samples with different GdN thicknesses. The GdN samples were then capped with $30 \mathrm{~nm}$ of $\mathrm{GaN}$ for ex situ measurements. The layer thicknesses were measured using SEM, XRR and RBS. XRD was used to characterise the crystalline structure of the films. The electrical transport properties of the GdN films were also measured using the van der Pauw geometry. Magnetic properties were measured via SQUID and VSM.

\subsection{Thin film thickness measurements}

The amount of material deposited during the growth inside the UHV chamber is recorded using a quartz deposition crystal placed near the substrate. To calibrate the values obtained from the deposition rate controller (DRC), external measurements of the film thicknesses must be used; a number of independent thickness measurement techniques are used to verify the effectiveness of XRR, a characterisation method previously unused at VUW. Table 4.1 summarises all sample thickness measurements performed, compared to the thickness recorded by the DRC (given in arbitrary units). A conversion factor (Section 4.5.4) is used to estimate the thickness of the other GdN thin films with no external thickness measurements.

\subsubsection{XRR}

Six of the samples with total layer thickness $\sim 120 \mathrm{~nm}$ or lower on the fused silica substrate were measured using XRR. The experimental data and the simulated profiles for each respective sample are shown in Fig. 4.2. The main 


\begin{tabular}{|c|c|c|c|c|c|c|c|}
\hline \multirow[t]{2}{*}{ Sample } & \multirow{2}{*}{$\begin{array}{l}\text { DRC } \\
\text { GdN } \\
\text { (arb. unit) }\end{array}$} & \multicolumn{2}{|c|}{ XRR } & \multicolumn{2}{|c|}{ RBS } & \multirow{2}{*}{$\begin{array}{l}\text { SEM } \\
\text { Total } \\
(\mathrm{nm})\end{array}$} & \multirow{2}{*}{$\begin{array}{l}\text { GdN } \\
\text { tooling factor }\end{array}$} \\
\hline & & $\begin{array}{l}\text { GdN } \\
(\mathrm{nm})\end{array}$ & $\begin{array}{l}\mathrm{GaN} \\
(\mathrm{nm})\end{array}$ & $\begin{array}{l}\mathrm{GdN} \\
(\mathrm{nm})\end{array}$ & $\begin{array}{l}\mathrm{GaN} \\
(\mathrm{nm})\end{array}$ & & \\
\hline $\mathrm{GdN}_{1000}$ & 1000 & & & 310 & 30 & & 0.31 \\
\hline $\mathrm{GdN}_{500}$ & 500 & & & 150 & 30 & & 0.30 \\
\hline $\mathrm{GdN}_{300}$ & 300 & 91.3 & 33.7 & & & & 0.304 \\
\hline $\mathrm{GdN}_{200}$ & 200 & 64.3 & 28.8 & & & 100 & 0.3215 \\
\hline $\mathrm{GdN}_{100} *$ & $100^{*}$ & 58.1 & 53.5 & & & 110 & $0.581^{*}$ \\
\hline $\mathrm{GdN}_{70} *$ & $70^{*}$ & 42.3 & 50.0 & 40 & 45 & 95 & $0.604^{*}$ \\
\hline $\mathrm{GdN}_{250}$ & 250 & & & 75 & 55 & & 0.3 \\
\hline $\mathrm{GdN}_{200 \mathrm{~b}}$ & 200 & 60.6 & 58 & & & & 0.303 \\
\hline $\mathrm{GdN}_{100 \mathrm{~b}}$ & 100 & 29.0 & 29.4 & & & & 0.29 \\
\hline $\mathrm{GdN}_{2000}$ & 2000 & & & 713 & 30 & & 0.356 \\
\hline
\end{tabular}

Table 4.1: Table of GdN thin film samples with external layer thickness measurements via XRR, RBS and cross-section SEM. Uncertainties for the respective measurement techniques for each layer are estimated to be $\pm 2 \mathrm{~nm}$ for XRR and $\pm 10 \mathrm{~nm}$ for RBS and SEM. The two starred samples were measured with a different deposition crystal located further away from the Gd source inside the chamber resulting in a larger tooling factor. 


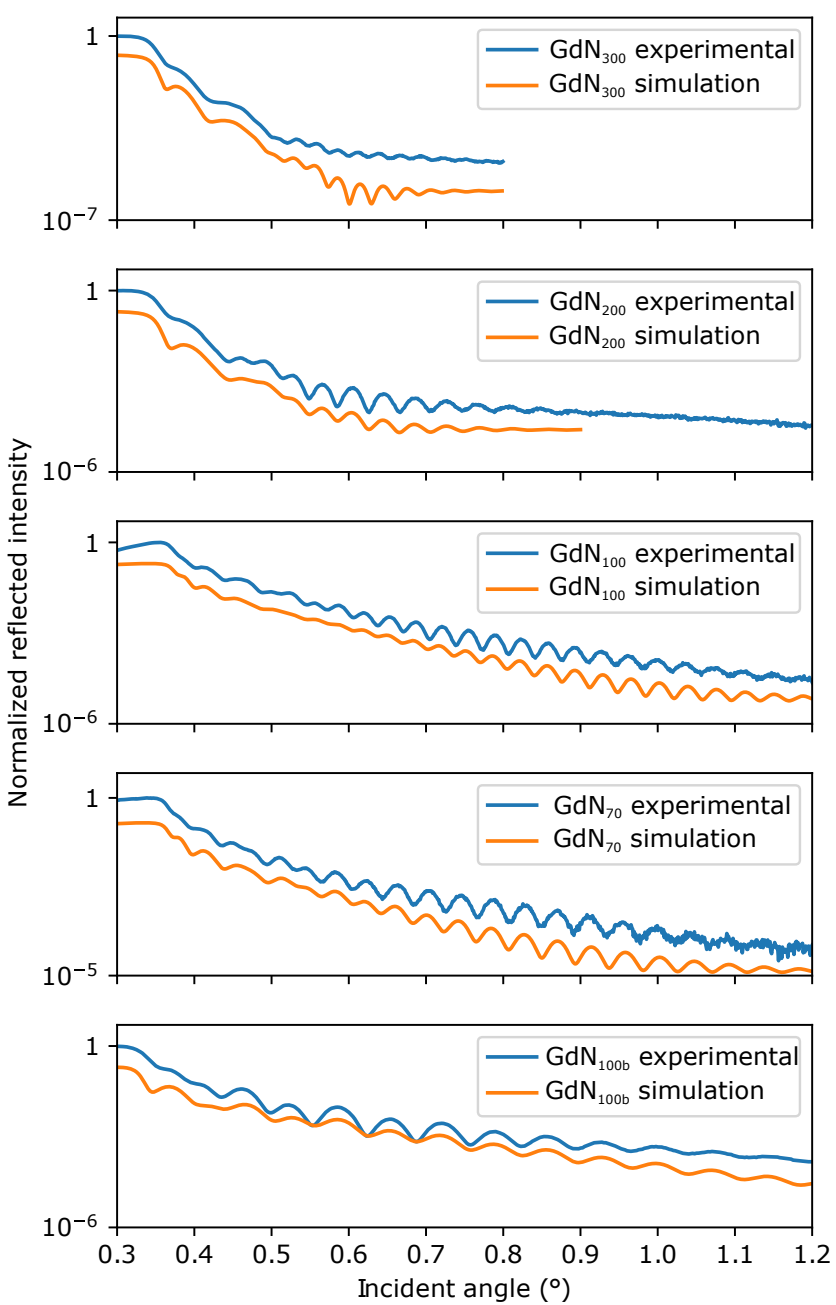

Figure 4.2: XRR measurements on GdN samples capped with GaN grown on fused silica substrates. The experimentally recorded reflected intensity as a function of incident angle is shown in blue next to the simulated reflectivity curves, offset for clarity. The differences between the fringe patterns of the different samples is caused by the interference between the layers of varying thicknesses, requiring simulated patterns from model structures to accurately extract the layer thicknesses. The layer thicknesses used to produce the simulated curves are shown in Table. 4.1 
parameters to be extracted, the thicknesses of the GdN and GaN layers are summarised in Table. 4.1.

\subsubsection{RBS}

Five of the samples were also measured using RBS. The measurements and fits were performed by Peter Murmu at GNS. The measured profiles and simulated fits are shown in Fig. 4.3. The Gd areal density is converted into an equivalent GdN film thickness using the density of GdN $\left(3.24 \times 10^{22} \mathrm{Gd} / \mathrm{cm}^{3}\right)$, summarised in Table. 4.1. The anomalous peak at $1.4 \mathrm{MeV}$ in the spectrum of sample $\mathrm{GdN}_{1000}$ does not match any elements in the deposited layers $(\mathrm{GdN}, \mathrm{GaN})$ nor the substrate $\left(\mathrm{SiO}_{2}\right)$ and may indicate contamination, possibly introduced before the RBS measurement.

\subsubsection{SEM}

Cross-section SEM was also performed on three of the samples (Fig. 4.4) to measure the film thicknesses. Poor contrast due to the insulating fused silica substrate allowed only the total thickness of the deposited layers ( $\mathrm{GdN} / \mathrm{GaN})$ to be extracted; the values are included in Table 4.1. The white layer present on the top surface of the samples $\mathrm{GdN}_{200}$ and $\mathrm{GdN}_{100}$ have been excluded as likely surface contamination on top of the GaN capping layer.

\subsubsection{Tooling factor}

A summary of external measurements of layer thicknesses is shown in Table 4.1. The deposition rate during film growth is measured using a quartzcrystal deposition rate controller (DRC) located inside the chamber near the substrate position. The tooling factor $F_{m}$, defined as the ratio of the actual film thickness $T_{\text {actual }}$ and the thickness value as measured by the DRC $T_{D R C}$, i.e.

$$
F_{m}=T_{\text {actual }} / T_{D R C}
$$




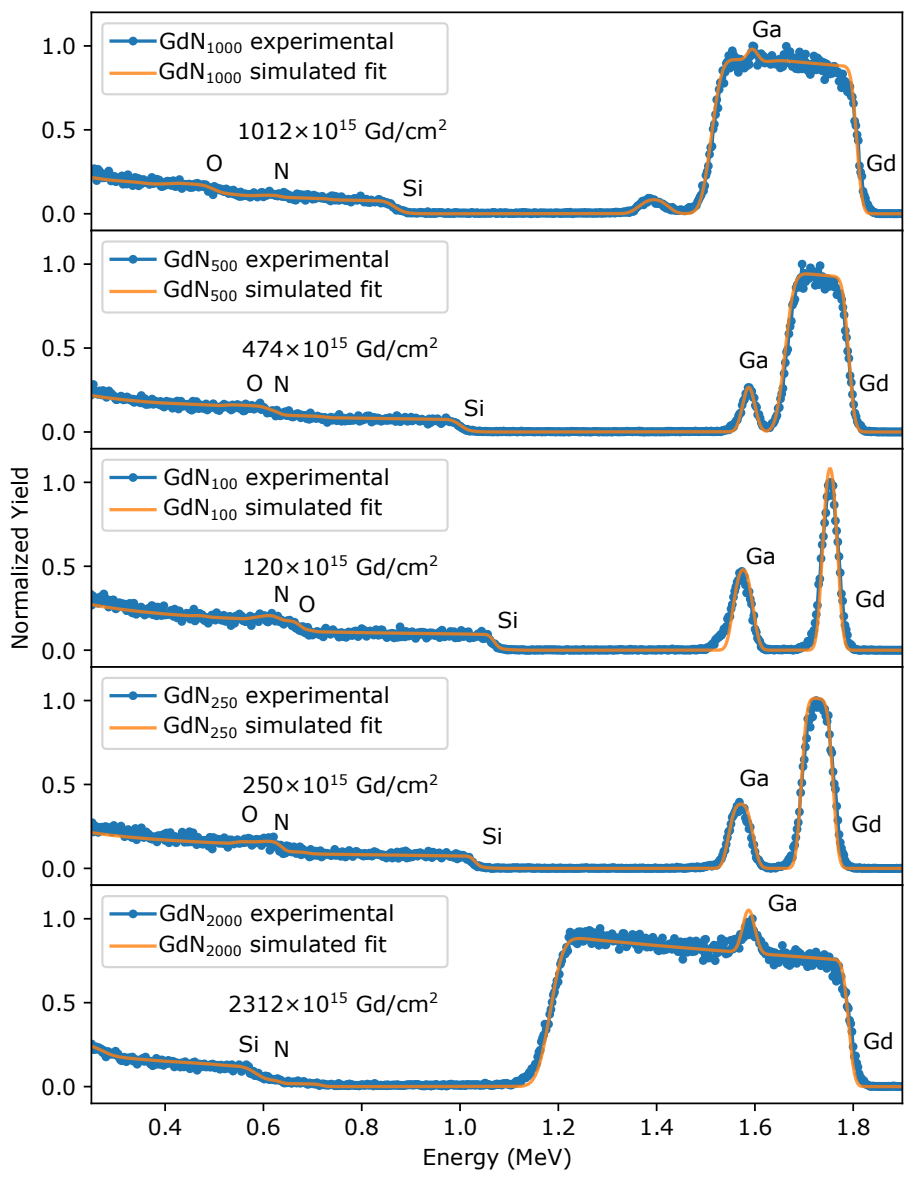

Figure 4.3: RBS profiles and simulated fits used to determine the elemental depth profile of selected GdN thin film samples. The total Gd atom areal density extracted from each fitting is shown and converted to a GdN film thickness in Table 4.1. 


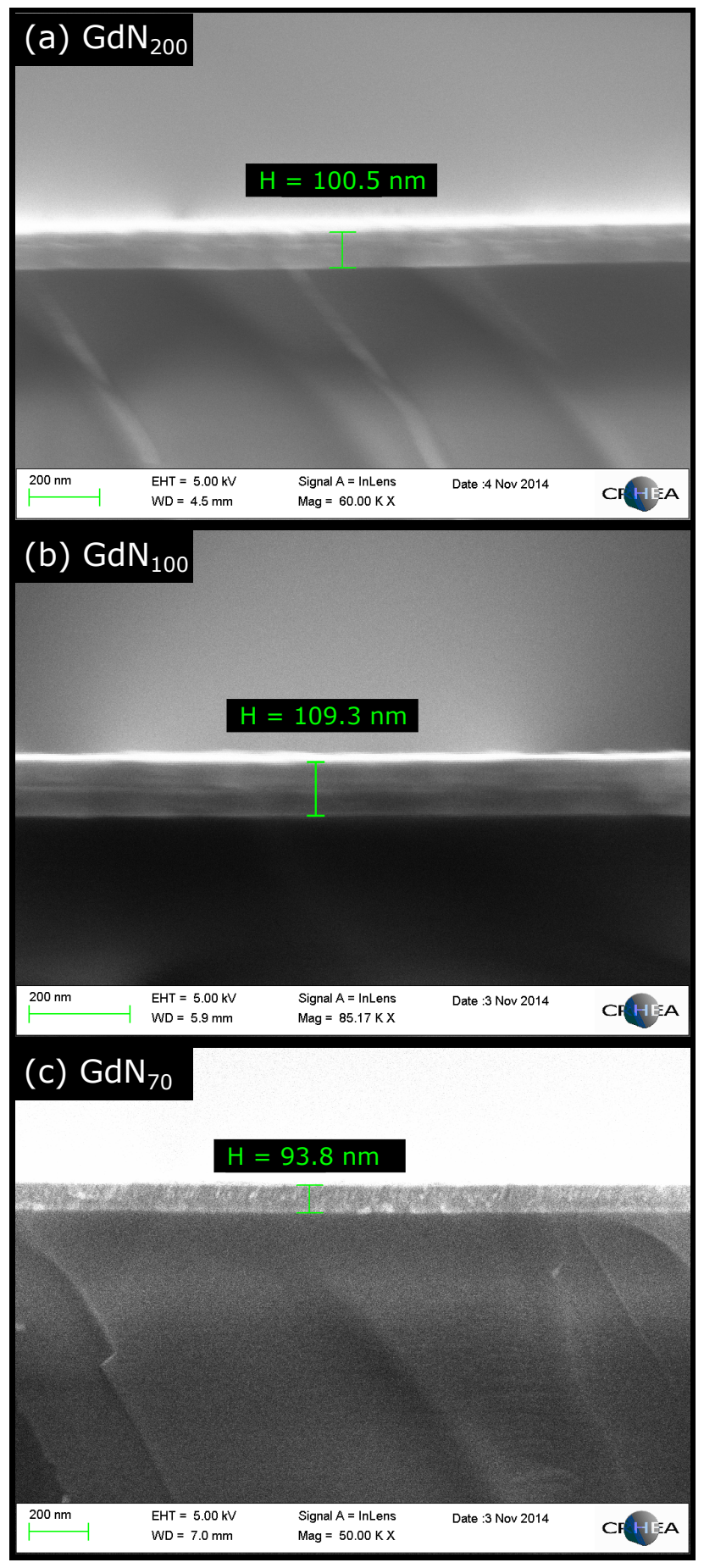

Figure 4.4: Cross-section SEM images of three GdN thin film samples. The total thickness of the GdN film and GaN capping layer is shown. 
is calculated for all measured samples, and an average value of $F_{m}=0.31$ used for all samples whose thickness was not explicitly measured

\subsection{Gd and nitrogen flux ratio}

Two important growth parameters for the deposition of GdN thin films are the incident fluxes of film precursors. These values cannot be directly measured in the UHV growth chamber used and are inferred indirectly. The average Gd flux can be calculated from the GdN film thickness as determined in Section 4.5 divided by the growth time. This is commonly expressed in units of $\mathrm{nm} \mathrm{h}^{-1}$ or $\mathrm{nm} \mathrm{s}^{-1}$. Multiplying by the atomic density of Gd in GdN (4 Gd atoms per cubic unit cell), approximately $32.4 \mathrm{Gd} / \mathrm{nm}^{3}$ gives the $\mathrm{Gd}$ flux in units of atoms per unit area per unit time. For example, a film growth rate of $0.1 \mathrm{~nm} \mathrm{~s}^{-1}$ or $360 \mathrm{~nm} \mathrm{~h}^{-1}$ corresponds to a Gd flux of $3.24 \mathrm{Gd} / \mathrm{nm}^{2} / \mathrm{s}$.

The flux of $\mathrm{N}_{2}$ molecules is inferred from the background pressure recorded during growth. The following formula for the impingement rate $\Gamma$ is derived from the ideal gas law:

$$
\Gamma=\frac{P}{\left(2 \pi m K_{B} T\right)^{\frac{1}{2}}}
$$

where $P$ is the pressure of the gas, $m$ the mass of the gas molecule, $K_{B}$ the Boltzmann constant and $T$ the temperature. If the pressure of $\mathrm{N}_{2}$ is given in mbar and using a temperature of $300 \mathrm{~K}$, the molecular flux of nitrogen in units of $\mathrm{N}_{2} / \mathrm{nm}^{2} / \mathrm{s}$ is simply

$$
\Gamma_{\mathrm{N}_{2}}=\left(2.87 \times 10^{6} \mathrm{~N}_{2} / \mathrm{nm}^{2} / \mathrm{s} / \mathrm{mbar}\right) P
$$

For example, a $\mathrm{N}_{2}$ background pressure recorded during film deposition of $5 \times 10^{-5}$ mbar corresponds to a $\mathrm{N}_{2}$ flux of $143.7 \mathrm{~N}_{2} / \mathrm{nm}^{2} / \mathrm{s}$

A plot of the calculated $\mathrm{N}_{2}$ and Gd fluxes of the samples grown for this study is shown in Fig. 4.5. The typical GdN growth rate and $\mathrm{N}_{2}$ pressure during deposition was $0.1 \mathrm{~nm} \mathrm{~s}^{-1}\left(3.24 \mathrm{Gd} / \mathrm{nm}^{2} / \mathrm{s}\right)$ and $5.0 \times 10^{-5} \mathrm{mbar}$ $\left(143.7 \mathrm{~N}_{2} / \mathrm{nm}^{2} / \mathrm{s}\right.$, although a few samples were grown at lower $\mathrm{N}_{2}$ pressure 


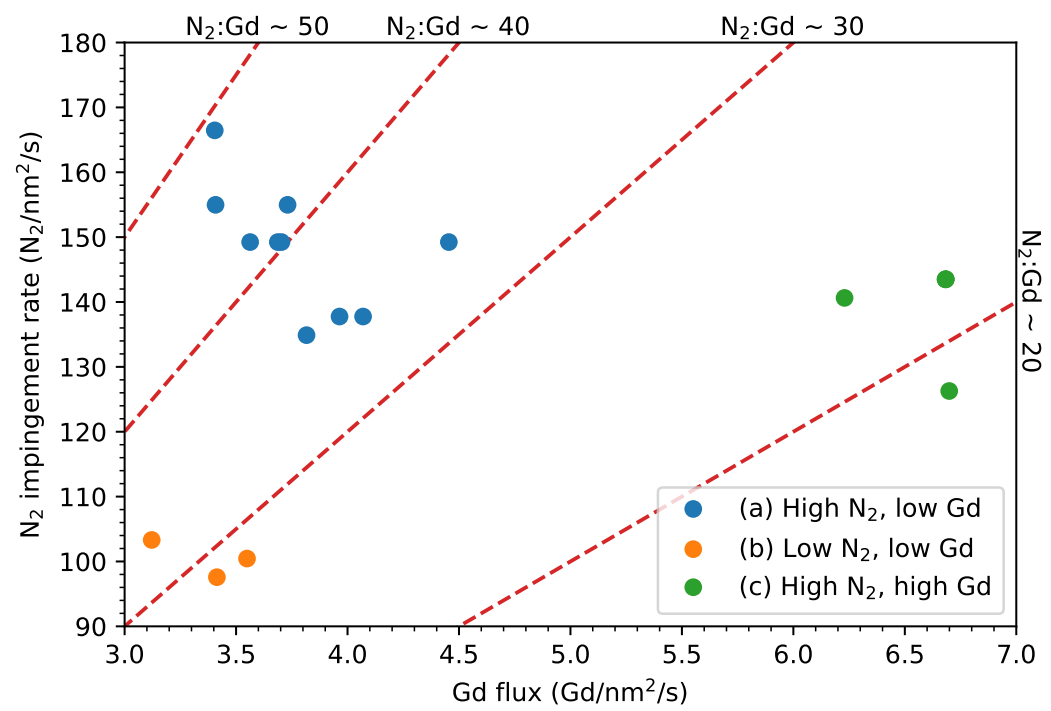

Figure 4.5: $\mathrm{N}_{2}$ and Gd fluxes for polycrystalline GdN samples grown for this study. The samples are roughly divided into 3 groups depending on relatively high or low $\mathrm{Gd}$ and/or $\mathrm{N}_{2}$ fluxes were used during deposition. The red dashed lines indicate fluxes that would correspond to the labelled $\mathrm{N}_{2}: \mathrm{Gd}$ ratios. 


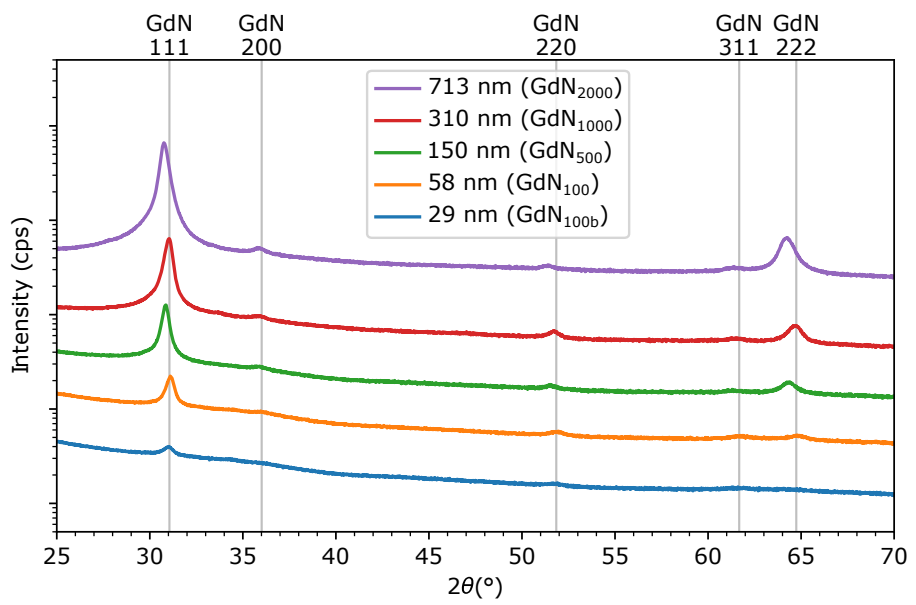

Figure 4.6: XRD scans of GdN samples with a range of GdN layer thicknesses showing the evolution of the diffraction peaks (offset for clarity). The expected peak centers for a rock salt GdN structure with a lattice constant of $a=4.98 \AA$ are shown.

$\left(\sim 3.2 \times 10^{-5}\right.$ mbar or $\sim 90 \mathrm{~N}_{2} / \mathrm{nm}^{2} / \mathrm{s}$ (b) or with higher Gd fluxes $\left(0.2 \mathrm{~nm} \mathrm{~s}^{-1}\right.$ or $6.5 \mathrm{Gd} / \mathrm{nm}^{2} / \mathrm{s}$ (c) as shown, and the results presented below will specify the sample group if relevant.

\subsection{Thickness dependence of GdN}

\subsubsection{XRD}

A selection of XRD scans of samples of GdN with varying thickness is shown in Fig. 4.6. Within the $2 \theta$ scan range of $25^{\circ}$ to $70^{\circ}$, polycrystalline GdN is expected to have peaks corresponding to the $111,200,220,311$ and 222 reflections, and these are evident in all samples above the amorphous background of the fused silica substrate. No peaks due to any impurity phases such as metallic Gd are visible, as can be observed in some Gdrich samples 19, 53, nor from the thin nanocrystalline GaN capping layer. As GdN film thickness increases, only the intensities of the 111 and 222 
reflections are seen to increase while the 200220 and 311 peaks do not vary significantly between samples. Comparing the peak areas of the most significant peaks, the 111 and 200 reflections, we conclude that, using the growth parameters as described above, GdN exhibits a preferential growth orientation in the [1 111 ] direction perpendicular to the substrate. The volume fraction $V_{f}$ of $\operatorname{GdN}(111)$ crystallites compared to $(001)$ crystallites that are oriented parallel to the sample surface can be estimated assuming the diffracted peak area is proportional to the diffracting volume of correctly oriented crystallintes within the film, using the equation

$$
V_{f}=\frac{I_{(111)} / S_{(111)}}{I_{(200)} / S_{(200)}+I_{(111)} / S_{(111)}}
$$

where $I_{(111)}$ and $I_{(200)}$ are the integrated areas of the 111 and 200 reflections divided by the structure factors $S_{(111)}$ and $S_{(200)}$ resulting from a uniformly randomly oriented (powder) sample. For GdN, the theoretical structure factors are $S_{(111)}=1$ and $S_{(002)}=0.7409$.

A plot of the volume fraction as a function of sample thickness is shown in Fig 4.7. The thinnest GdN sample measured with a layer thickness of $30 \mathrm{~nm}$ has a $V_{f,(11)}=0.67$ and this is seen to approach 1 asymptotically as film thickness increases up to the thickest film measured. This demonstrates that the growth of polycrystalline GdN thin films at room temperature tends to favour the (111) orientation parallel to the substrate even starting from an amorphous substrate. This preferential orientation seems to start in the first tens of nm of deposition and intensifies as the deposition continues up to the thickest films grown at $700 \mathrm{~nm}$. This is consistent with the evolutionary selection model (Section 4.2) showing that GdN grows fastest along the (1 11 ) crystal face during deposition and this growth direction dominates over other orientations early in the film growth. Additionally, this shows that nucleation of crystallites oriented in other directions does not occur in any significant volume on top of the (111) growth front up to the maximum thickness explored.

A plot of the average lattice constant of the GdN in the thin film samples 


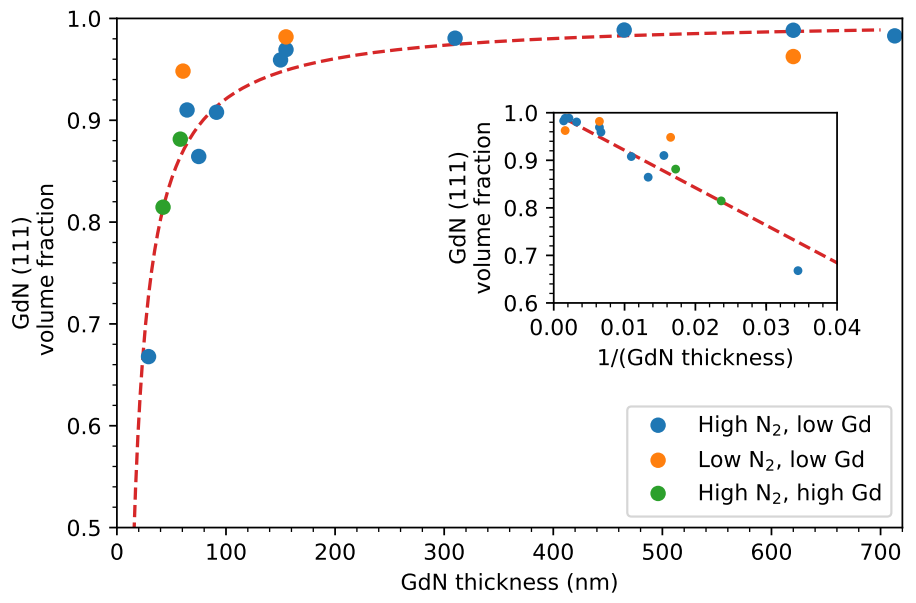

Figure 4.7: Volume fraction of GdN (111) crystallites oriented parallel to the sample surface compared to $(200)$-oriented crystallites. The red dashed line is a fit of the form $f(T)=1-c / T$, demonstrating the asymptotic approach to a fully (111)-oriented film. The inset plots the volume fraction against the inverse of film thickness.

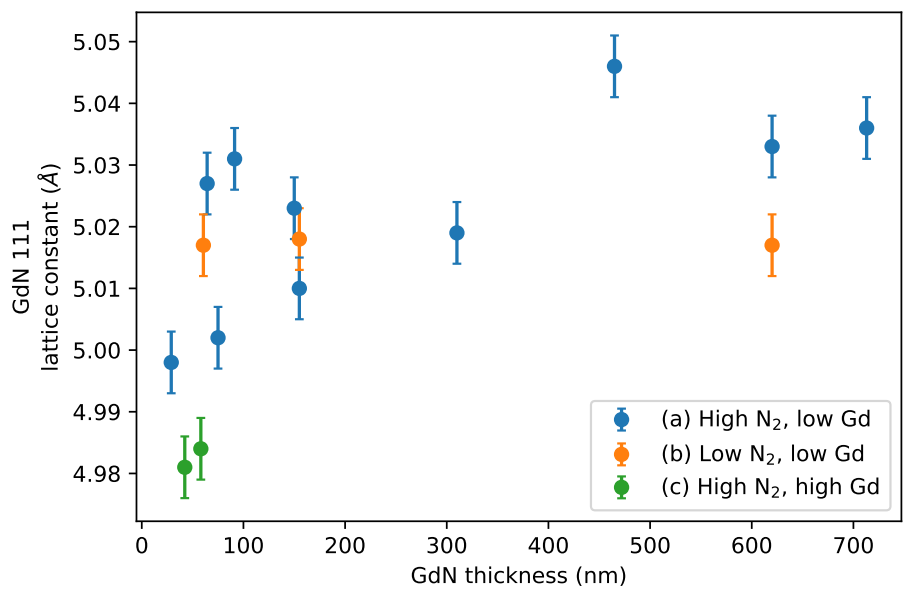

Figure 4.8: Plot of the lattice constant of GdN using the 111 peak location as a function of film thickness. The uncertainty for this measurement is estimated to be about $0.005 \AA$ for all films. 


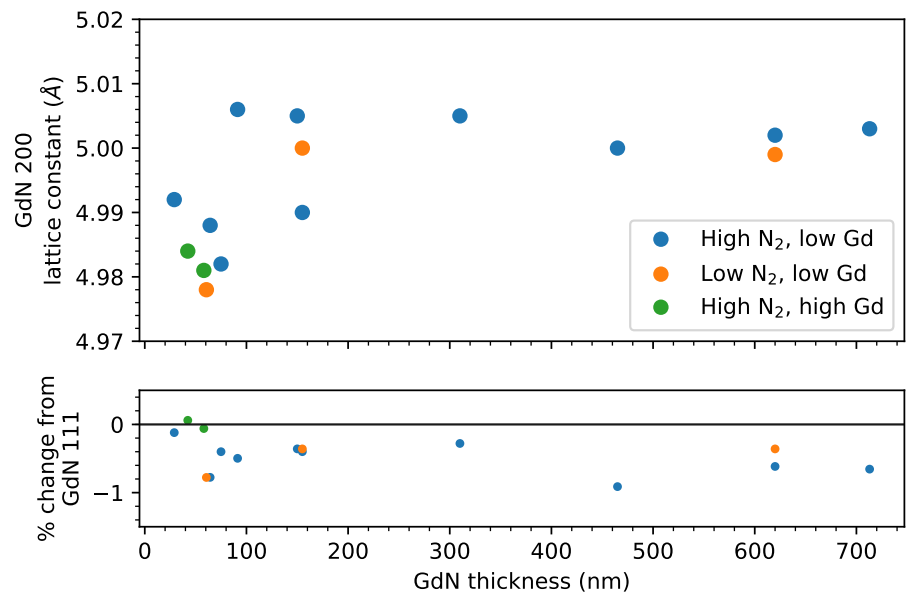

Figure 4.9: Plot of the lattice constant of GdN using the 200 peak location as a function of film thickness. The percentage change from the GdN 111 lattice constant is shown.

is shown in Fig. 4.8. The out-of-plane lattice constant $a$ is determined from the 111 peak centre, using Bragg's law and assuming a cubic symmetry, i.e.

$$
a=\sqrt{\frac{h^{2}+k^{2}+l^{2}}{d_{h k l}}}=\sqrt{\frac{h^{2}+k^{2}+l^{2}}{\frac{\lambda}{2 \sin \theta}}}
$$

with $h=k=l=1$. The extracted lattice constants vary quite significantly between $4.98 \AA$ to $5.04 \AA$ between different samples, with thinner samples tending to have smaller lattice constants, though this correlation is not strong. The 111 peak is chosen because it is the most intense reflection, i.e. it represents the bulk of the GdN crystallites that are measured in this geometry, but if the 200 peak is used instead, the extracted lattice constant is smaller by an average of $0.4 \%$ (Fig. 4.9 . Note that due to the emergence of the preferential orientation as noted above, the 200 peak is much weaker due to most of those crystallites occurring close to the substrate/film interface.

These values for the lattice constant are comparable to other GdN films reported in the literature [7] but larger than the $4.984 \AA$ reported for single crystal GdN. A lattice expansion out-of-plane as is being measured with 


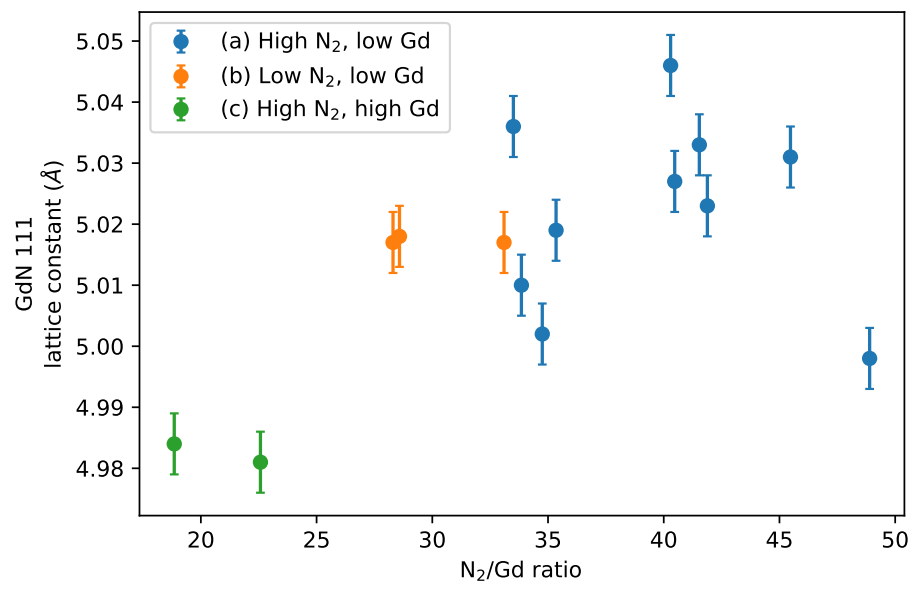

Figure 4.10: Plot of the out-of-plane lattice constant of GdN using the 111 peak location as a function of $\mathrm{N}_{2} / \mathrm{Gd}$ ratio during deposition.

the Bragg-Brentano XRD geometry might suggest a compressive stress inplane in the film. The various origins of compressive stress generation in polycrystalline thin films are widely debated, but one relevant possibility is the insertion of adatoms into grain boundaries [54]. In addition to the weak correlation of larger lattice constant with film thickness in Fig. 4.8, the outof-plane lattice constant shows some dependence on the $\mathrm{N}_{2} / \mathrm{Gd}$ ratio during deposition (Fig. 4.10), with larger lattice constants observed for growths at higher $\mathrm{N}_{2} / \mathrm{Gd}$ ratios. It is possible that interstitial $\mathrm{N}$ or $\mathrm{N}_{2}$ inserted into the grain boundaries between the columnar grains induces a compressive stress in-plane causing the observed expanded lattice constants out-of-plane.

The broadening $\beta_{T}$ of the diffraction lines resulting in peaks has an instrumental contribution $\beta_{\text {inst }}$ and a sample contribution $\beta_{\text {sample } 55 .}$. The instrumental broadening is an artefact from the chosen optical and geometrical setup and is approximately $0.04^{\circ}$ for the XRD measurements performed, much smaller than the sample broadening observed. The sample broadening is due to deviations from a perfect infinite crystal, namely:

- Crystallite size (diffraction coherence length) 


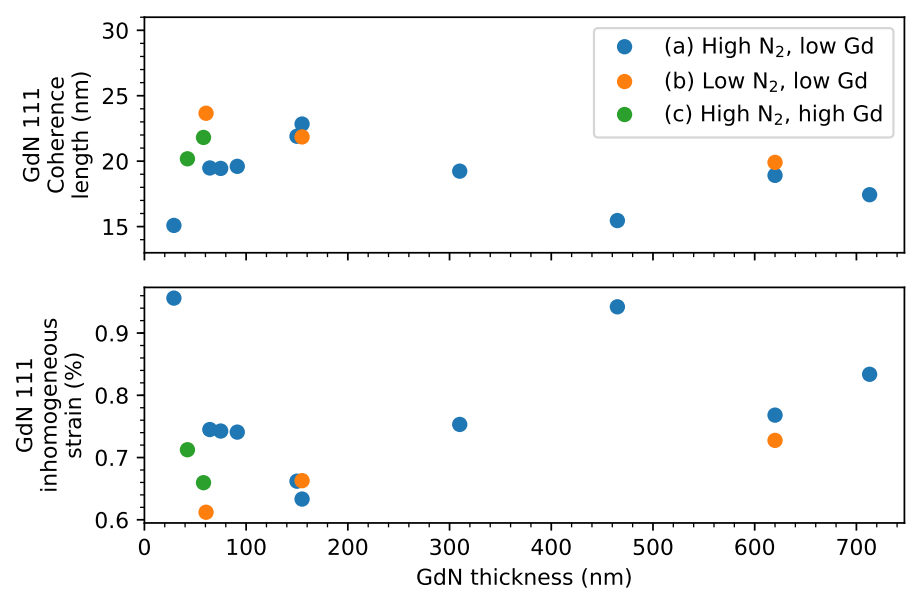

Figure 4.11: Estimates for the volume-weighted coherence length and inhomogeneous strain assuming the peak broadening is solely due to each respective factor. The values for the coherence length and strain are lower and upper bounds respectively.

- Inhomogeneous strain effects

- Lattice defects (e.g. stacking/twin faults)

If the broadening is assumed to result entirely from size effects, the Scherrer equation gives the broadening of an observed peak as a function of the average crystallite size $L$

$$
\beta_{L}=\frac{K \lambda}{L \cos \theta}
$$

Alternatively, if the other extreme is considered with the broadening resulting entirely from inhomogeneous strain effects, the broadening has a dependence on the Bragg angle of

$$
\beta_{e}=C \varepsilon \tan \theta
$$

where $C$ is a constant depending on the nature of the strain, but generally assumed to be between 4 and 5 .

The Williamson-Hall method takes advantage of the different angular dependences of both sources of broadening; assuming the combined broadening 


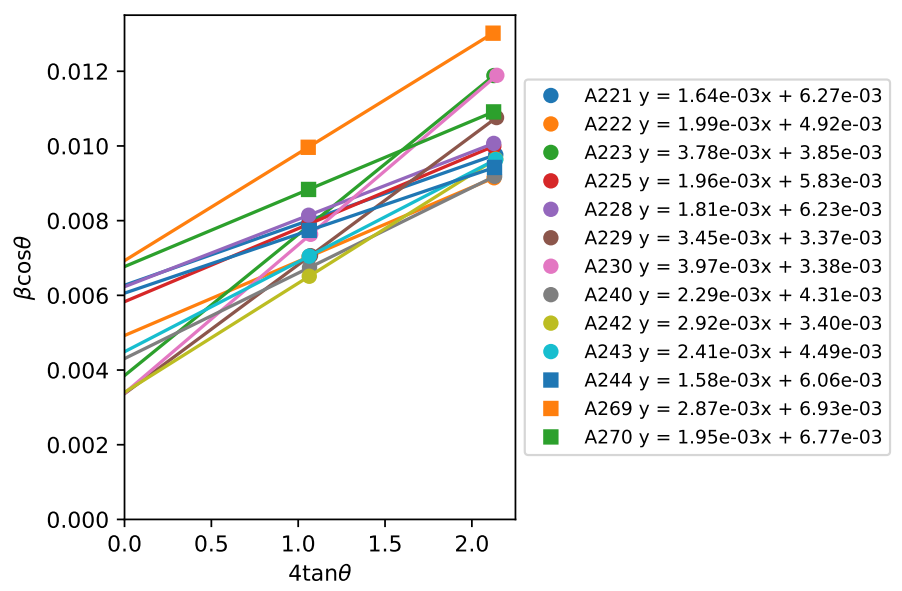

Figure 4.12: Williamson-hall plot using the GdN 111 and 222 peaks in the XRD scans

is a simple sum of the two (i.e. the convolution of Lorentzian functions), then the broadening is

$$
\begin{aligned}
\beta=\beta_{e}+\beta_{L} & =C \varepsilon \tan \theta+\frac{K \lambda}{L \cos \theta} \\
\beta \cos \theta & =C \varepsilon \sin \theta+\frac{K \lambda}{L}
\end{aligned}
$$

and a plot of $\beta \cos \theta$ against $C \sin \theta$ for multiple peaks allows the strain $\varepsilon$ and size $L$ to be extracted from a linear fit from the gradient and y-intercept.

A Williamson-Hall plot using only the GdN 111 and 222 diffraction peaks is shown in Fig. 4.12. These peaks represent the bulk of the film for the used XRD geometry, although there is no error in fitting a line to two points. The extracted coherence lengths and inhomogeneous strain values are plotted as a function of GdN thickness in Fig. 4.13 as well as the ratio with the values assuming only size or strain broadening. Thinner films tend to have a more even mix of size and strain broadening, so that the $\mathrm{W}-\mathrm{H}$ extracted coherence length is nearly double that if only size broadening is assumed; similarly the strain value is halved for the thinnest films. Conversely, thicker films have a smaller contribution from inhomogeneous strain and most of the 

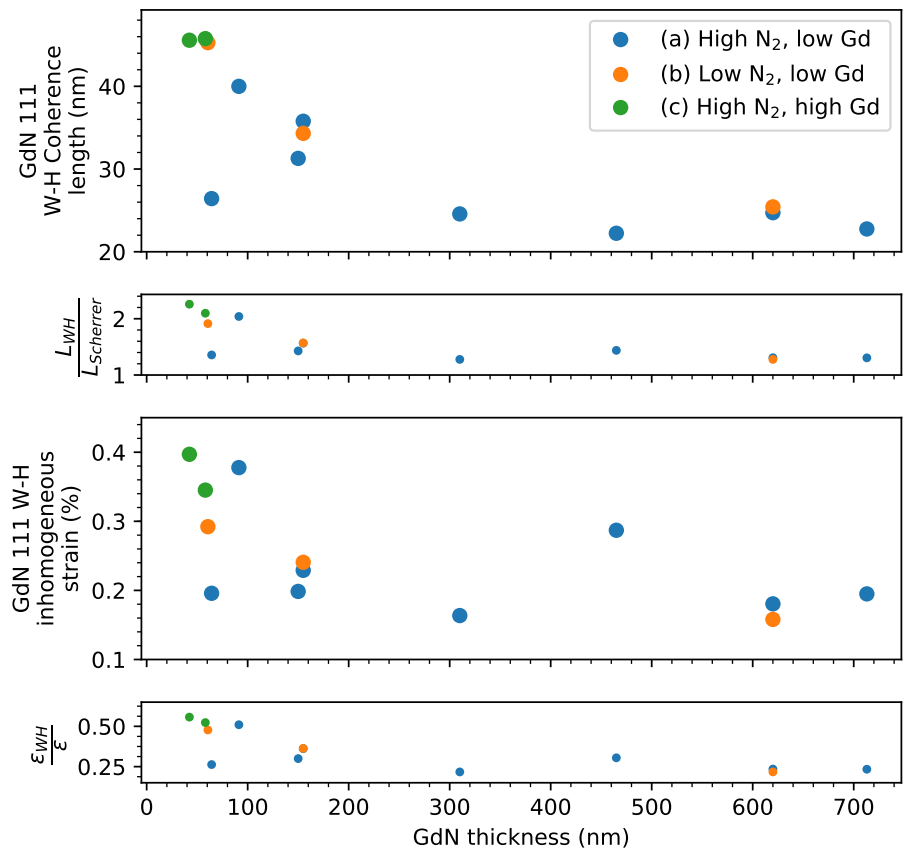

Figure 4.13: Volume-weighted coherence length and inhomogeneous strain as extracted from the Williamson-Hall plot (Fig. 4.12) intercepts and gradients. The ratio between the values obtained from $\mathrm{W}-\mathrm{H}$ and those from fully size or strain induced broadening are also plotted respectively. 


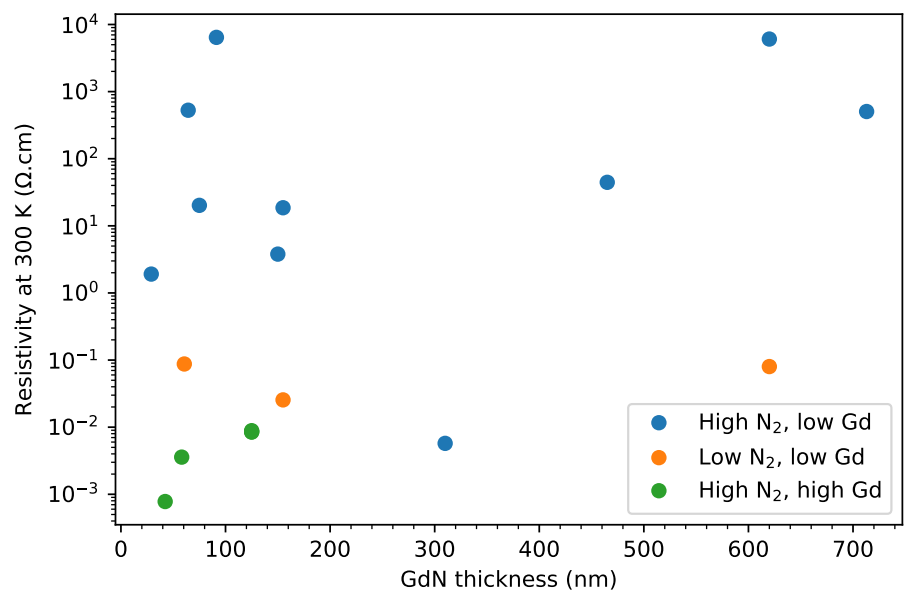

Figure 4.14: Resistivity of GdN measured at room temperature $(300 \mathrm{~K})$ as a function of GdN layer thickness.

peak broadening is due to the size of the coherently diffracting crystallites.

The calculated coherence length decreases with increasing thickness which is unusual in light of the evolutionary selection model presented in Section 4.2 . the coherence length of the thinnest film with a GdN thickness measured to be $42 \mathrm{~nm}$ is calculated to be $45 \mathrm{~nm}$ (shape factor $K$ assumed to be 1 for needle-like crystallites, might be too large). The coherence length decreases with increasing GdN thickness and settles between $20 \mathrm{~nm}$ to $25 \mathrm{~nm}$ for films greater than $200 \mathrm{~nm}$ in thickness.

Inhomogeneous strain is similarly largest for the thinnest film with a maximum value of $0.4 \%$ and also decreases with increasing GdN thickness.

\subsubsection{Electrical transport}

We turn to look at the electrical transport properties of the GdN thin film samples. Temperature dependent resistivity measurements from $300 \mathrm{~K}$ to $4 \mathrm{~K}$ were taken using the 4 -terminal van der Pauw geometry, contacting the sample through the insulating capping layer. A huge range of resistivities were observed ranging between $7 \times 10^{-4} \Omega \mathrm{cm}$ to $5 \times 10^{3} \Omega \mathrm{cm}$ at $300 \mathrm{~K}$ for 


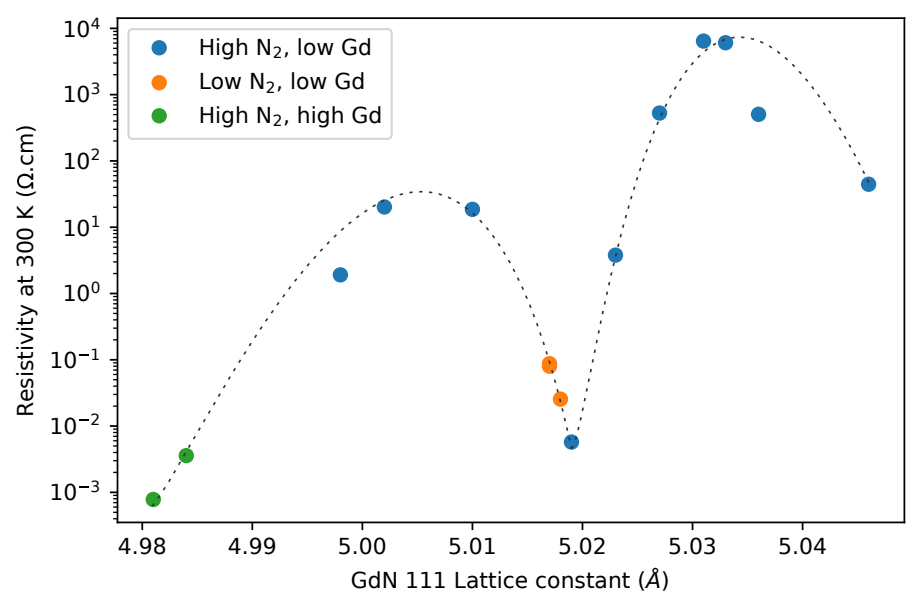

Figure 4.15: Resistivity of GdN measured at room temperature $(300 \mathrm{~K})$ as a function of GdN lattice constant.

different samples, spanning nearly 7 orders of magnitude. A plot of the room temperature resistivity as a function of GdN thickness shows no obvious correlation (Fig. 4.14). However, when the measured resistivity is compared to the lattice constant extracted from XRD measurements above, a visible trend seems to emerge (Fig. 4.15).

A local minimum in the resistivity seems to appear around a lattice constant of $5.018 \AA$, where the resistivity is measured to be between $10^{-2} \Omega \mathrm{cm}$ to $10^{-1} \Omega \mathrm{cm}$. As the lattice constant either increases or decreases around this point, the resistivity increases by some orders of magnitude. Further lattice expansion/contraction seems to result in the resistivity decreasing again, and the samples with the smallest resistivities below $10^{-2} \Omega \mathrm{cm}$ at $300 \mathrm{~K}$ have lattice constants smaller than $4.99 \AA$. It is reasonable to think that changes in the lattice constant could have strong effects on the band structure of the GdN bulk, but the factors potentially influencing changes in the lattice constant are not conclusive. It is known that the concentration of nitrogen vacancies in the GdN affects the resistivity strongly [19], and there has been speculation that nitrogen vacancies cause local lattice distortions possibly 
resulting in a separate phase of $\mathrm{GdN}$ [53] but both these results showed evidence of metallic Gd present in the N-deficient GdN which was not present in any of the samples grown for this study.

For the more conductive samples, it was possible to measure the carrier concentration via the Hall effect. An example temperature dependent Hall effect measurement is shown in Fig. 4.16. The carrier concentration is highest at $300 \mathrm{~K}$, at $1.5 \times 10^{21} \mathrm{~cm}^{-3}$ and steadily decreases with temperature to $0.6 \times 10^{21} \mathrm{~cm}^{-3}$ at $100 \mathrm{~K}$. Below this temperature, an anomalous Hall contribution emerges and the carrier concentration as extracted from the measurements at high field begins to increase again as the temperature approaches $5 \mathrm{~K}$. The hall mobility for this sample was calculated to be $0.7 \mathrm{~cm}^{2} /(\mathrm{V} \cdot \mathrm{s})$, with a maximum of $1.3 \mathrm{~cm}^{2} /(\mathrm{V} \cdot \mathrm{s})$ at $100 \mathrm{~K}$.

The carrier concentration and electron mobilities measured at $200 \mathrm{~K}$ are shown in Fig. 4.17. The samples with the lowest resistivities were grown with high Gd fluxes (Section 4.6) and this is reflected also in the highest carrier concentrations. The average electron mobility of about $1 \mathrm{~cm}^{2} /(\mathrm{V} \cdot \mathrm{s})$ is usual for a disordered polycrystalline GdN thin film; highly ordered epitaxial GdN thin films can exhibit mobilities in the range of $10 \mathrm{~cm}^{2} /(\mathrm{V} \cdot \mathrm{s})$ to $100 \mathrm{~cm}^{2} /(\mathrm{V} \cdot \mathrm{s}) 36$

\subsubsection{Magnetic properties}

The polycrystalline GdN samples were also measured using SQUID and VSM magnetometry in-plane. Zero-field-cooled/field-cooled (ZFC-FC) curves (temperature dependence) and magnetic hysteresis (field dependence) were measured down to $5 \mathrm{~K}$ as described in Section 3.7.2. An example SQUID measurement on one of the GdN samples is shown in Fig. 4.18 .

Eq. 3.48 is fit to the inverse susceptibility plotted against temperature to estimate $T_{C}$ and the number of Gd atoms that contribute to the ferromagnetic transition, assuming the $\mathrm{Gd}^{3+}$ effective magnetic moment as $\mu_{\text {eff }}=7.937 \mu_{B}$. The fraction of magnetic Gd compared to the total amount of $\mathrm{Gd}$ from the sample thickness is shown in Fig. 4.19 as a function of film thickness 

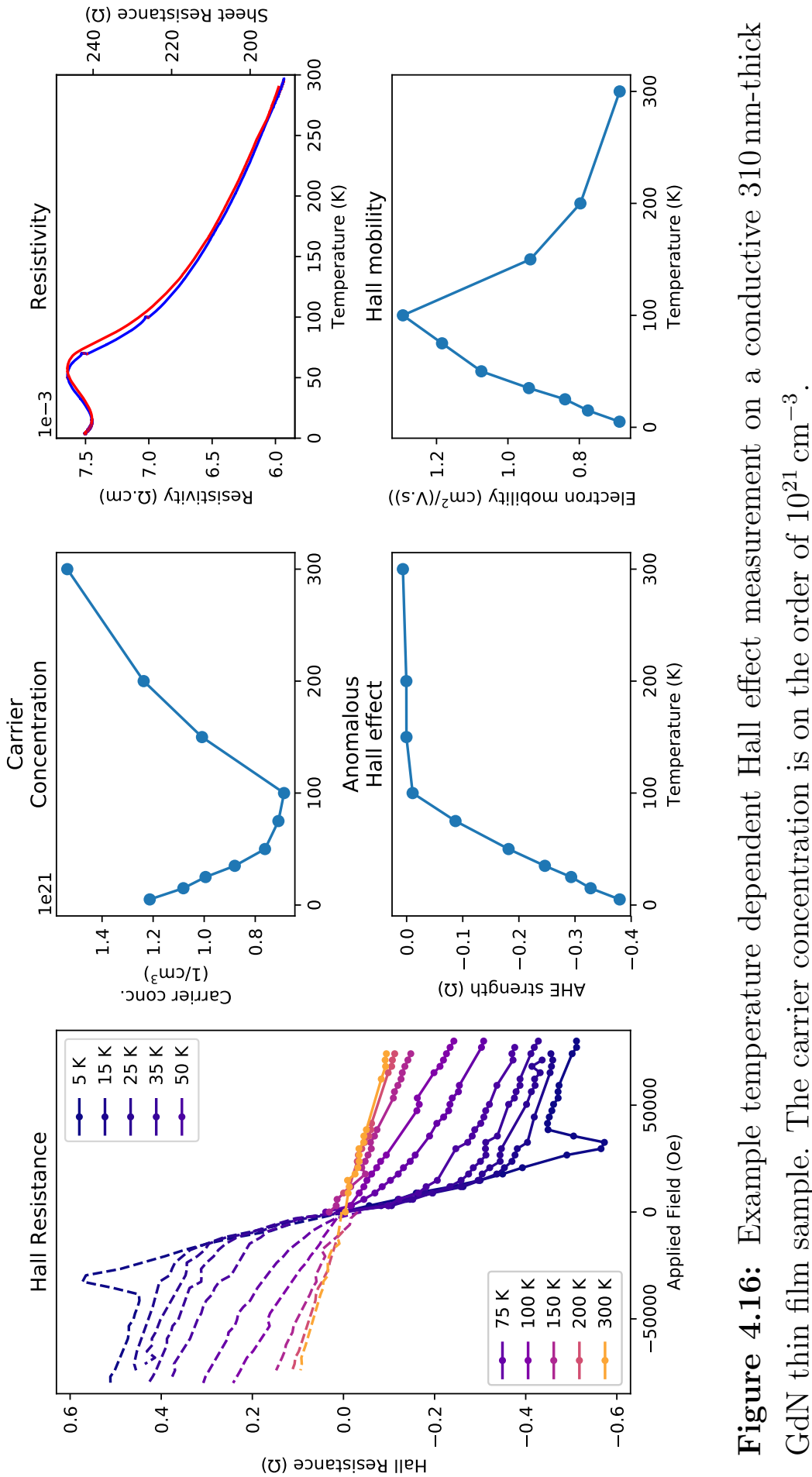

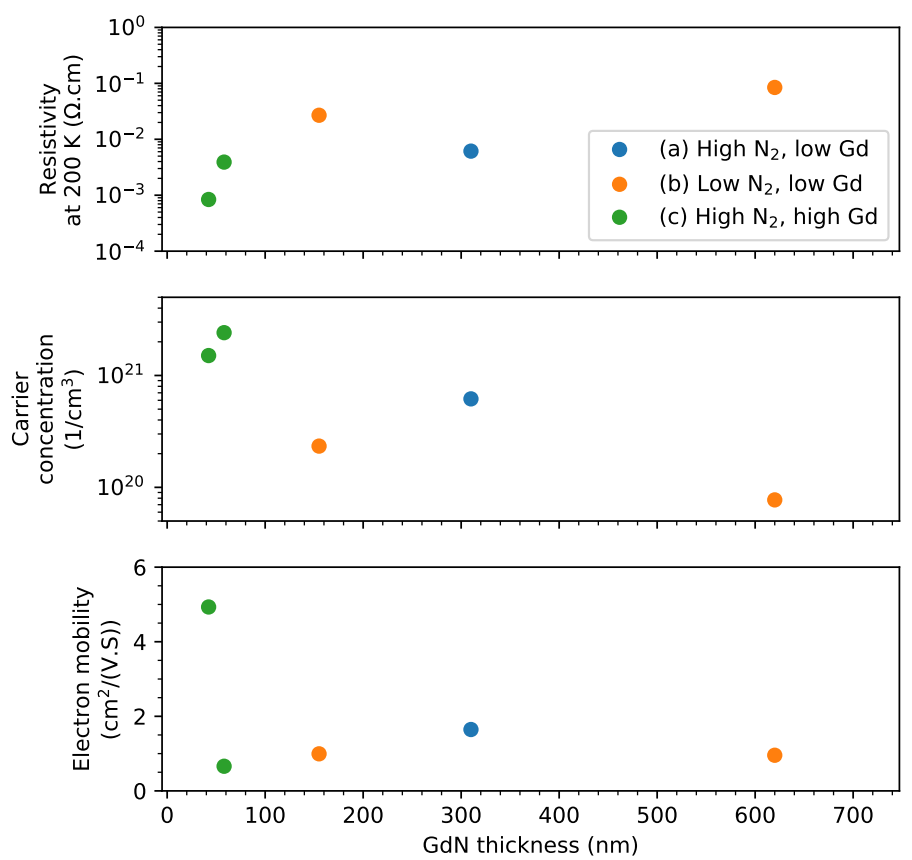

Figure 4.17: Carrier concentration and electron mobility measured for four samples of GdN at $200 \mathrm{~K}$ 

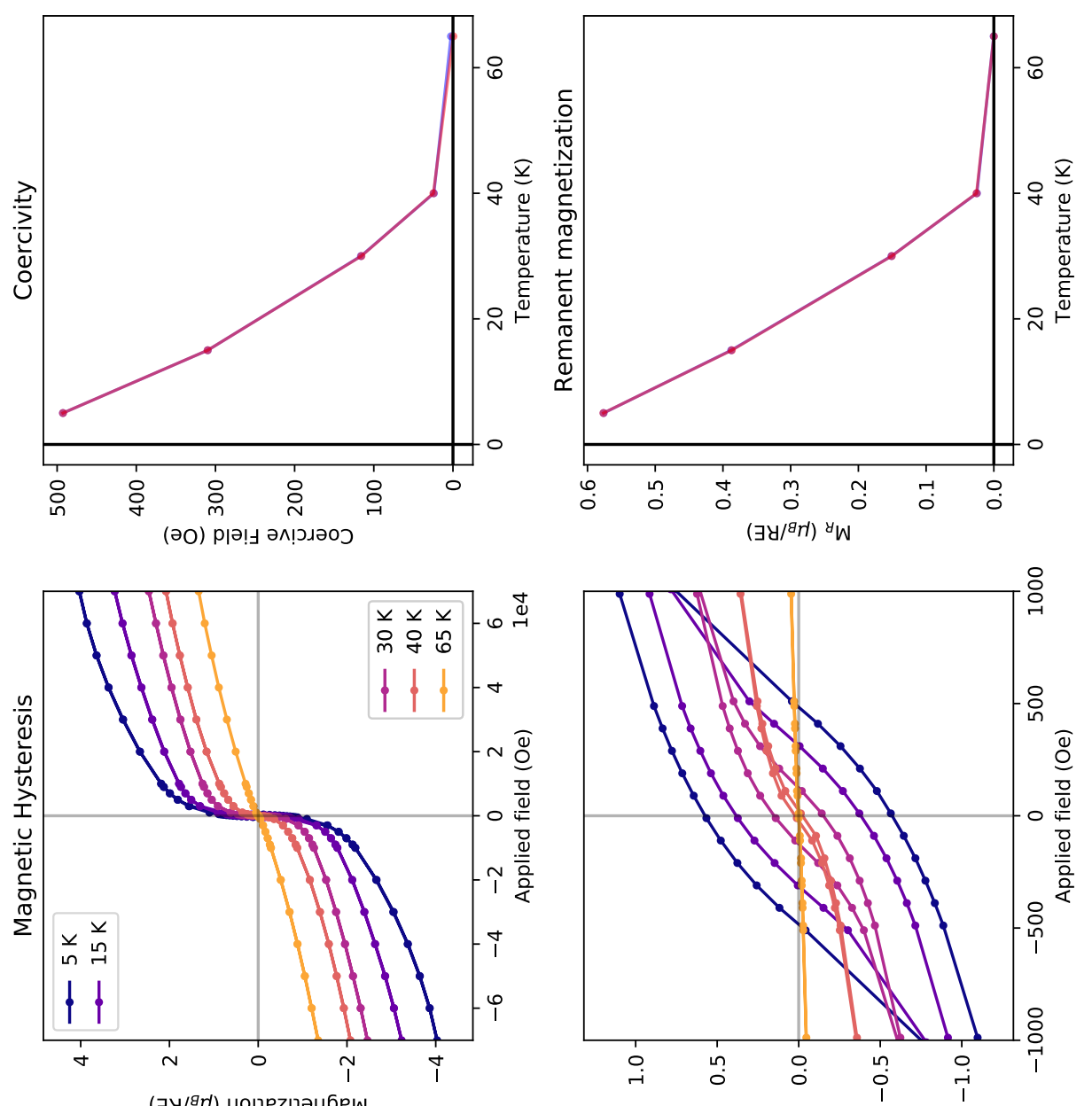

U

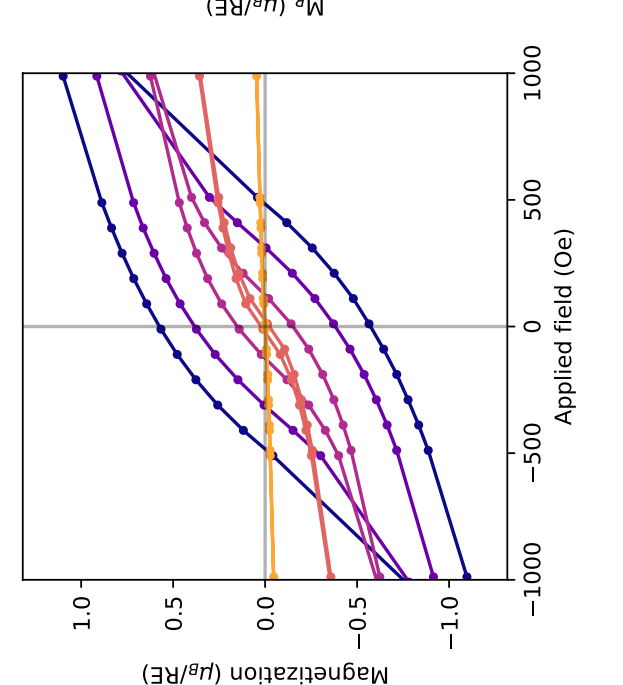

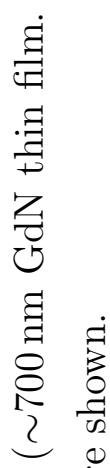

范

省

ㄱㅇ ஓ

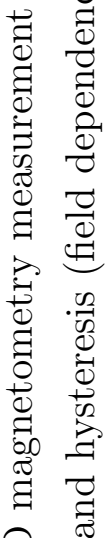
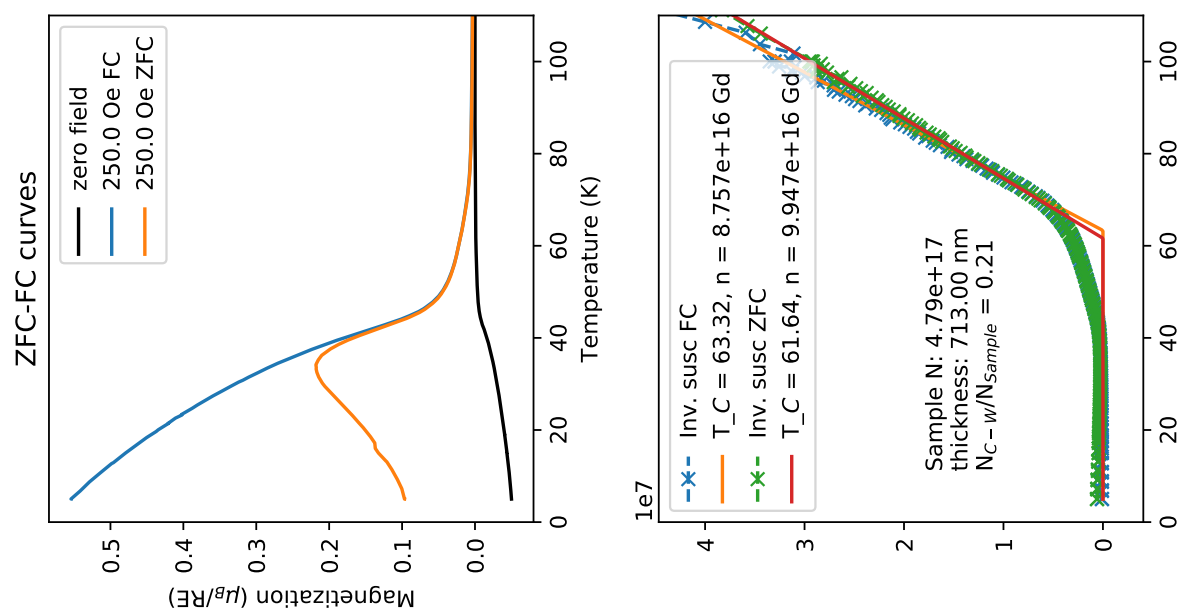

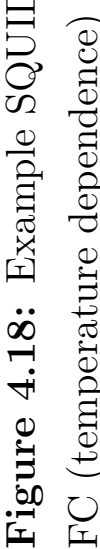




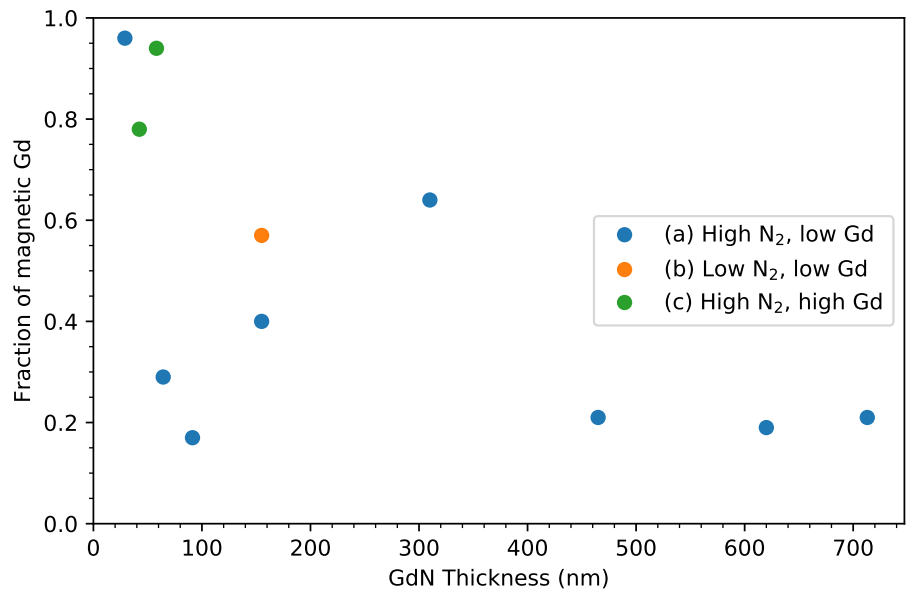

Figure 4.19: Fraction of magnetic $\mathrm{Gd}^{3+}$ ions as determined from the Curie-Weiss law as a function of GdN, ranging from above 0.9 for thin films below $60 \mathrm{~nm}$, to 0.2 for the thickest films.

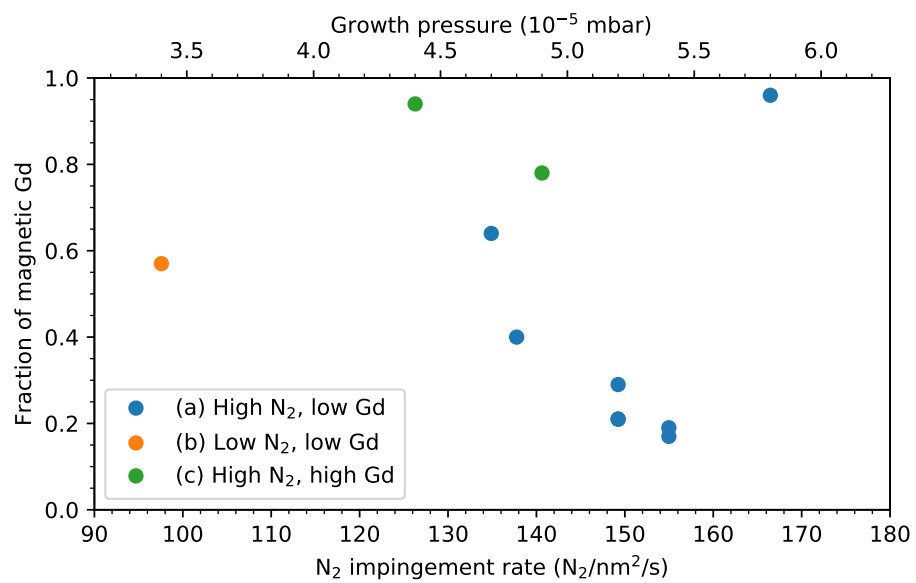

Figure 4.20: Fraction of magnetic $\mathrm{Gd}^{3+}$ ions as determined from the Curie-Weiss law as a function of $\mathrm{N}_{2}$ impingement rate. 


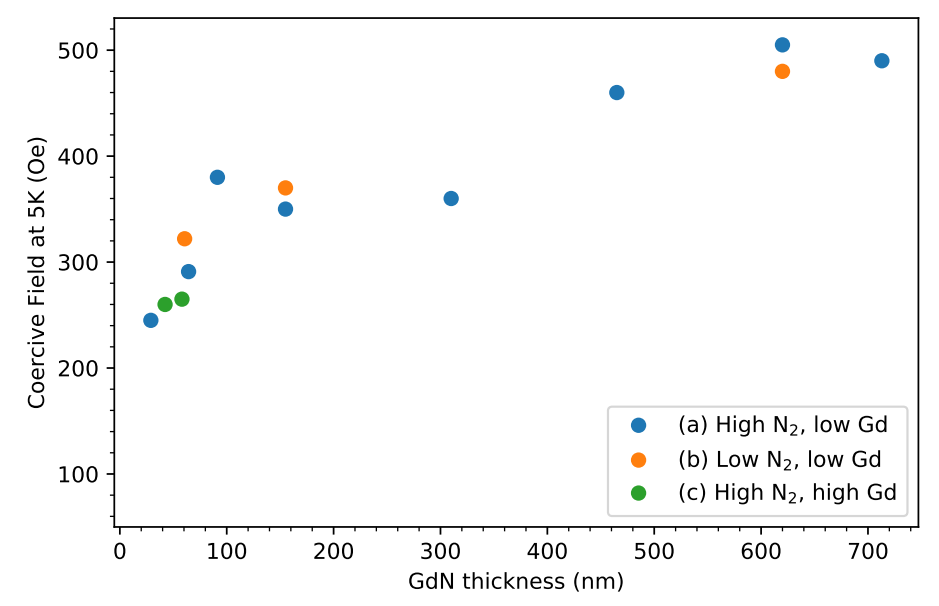

Figure 4.21: In-plane coercivity of GdN thin film measured at $5 \mathrm{~K}$ as a function of GdN thickness

and Fig. 4.20 as a function of $\mathrm{N}_{2}$ impingement rate. A small fraction of oxygen impurities is known to eliminate the ferromagnetic ordering of $\mathrm{GdN}$ resulting in paramagnetic or antiferromagnetic $\mathrm{GdN}_{0.95} \mathrm{O}_{0.05}[56$. Limited oxygen doping incorporated into the GdN films during growth is a likely factor resulting in the difficulty of saturating these polycrystalline films as well as the low fraction of magnetic Gd.

For hysteresis measurements, the sample is set to a fixed temperature below the magnetic transition temperature and the field cycled from $70000 \mathrm{Oe}$ to -70000 Oe and back up. The sample magnetisation is assumed to be saturated at the maximum applied field, and the sample remains magnetised as the external field is reduced to zero, giving the remanent magnetisation for that temperature. The coercive field or coercivity is defined as the field in the opposite direction required to further reduce the sample magnetisation to zero. The coercivity and remanent magnetisation are plotted as a function of temperature.

The coercivity of the GdN film as a function of thickness is shown in Fig. 4.21. The coercivity is smallest for the thinnest film (240 Oe at $30 \mathrm{~nm}$ ) 


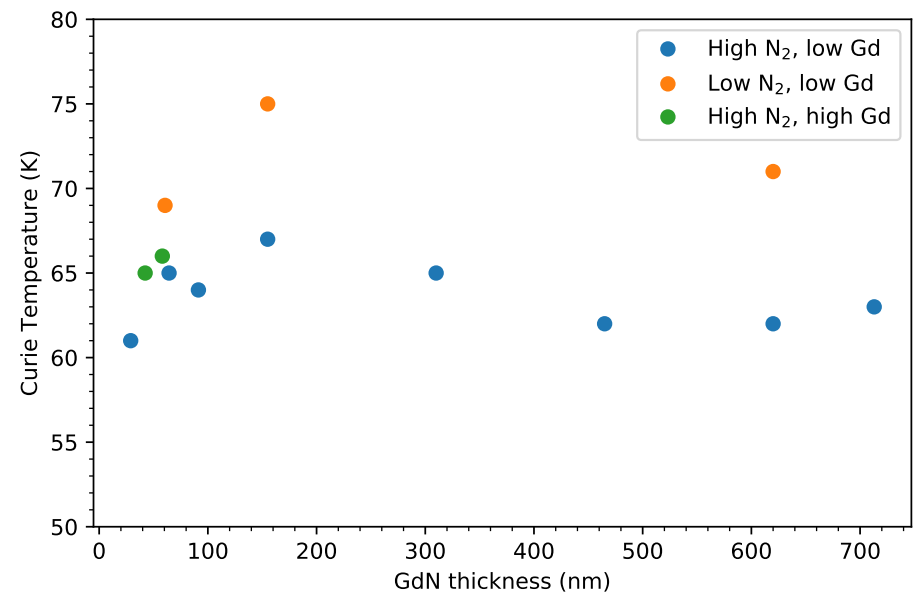

Figure 4.22: Curie temperature as determined from linear extrapolation of Curie-Weiss law plotted as a function of GdN thickness.

and increases with increasing film thickness, with a maximum coercivity of about 500 Oe observed in the thickest films. Similar thickness dependence of magnetic coercivity has been observed in other ferromagnetic thin films due to the dependence on the volume to surface ratio of these magnetic properties [57.

The Curie temperature for all samples is shown in Fig. 4.22 as a function of GdN thickness and Fig. 4.23 plotted against the $\mathrm{N}_{2}$ impingement rate. The measured Curie temperature is seen to vary between $61 \mathrm{~K}$ to $75 \mathrm{~K}$ and does not show any dependence on the overall GdN film thickness but does exhibit a strong dependence on the $\mathrm{N}_{2}$ impingement rate (growth pressure during deposition). This seems to be independent of the Gd flux (and consequently $\mathrm{N}_{2}$ /Gd ratio) which would suggest the magnetic transition temperature is not strongly dependent on the film microstructure but mainly on the stoichiometry. The independence of the curie temperature from the Gd flux suggests that the incorporation of $\mathrm{N}_{2}$ to approach stoichiometric GdN is not a simple linear relation of the ratios of the $\mathrm{Gd}$ and $\mathrm{N}_{2}$ fluxes.

The temperature dependence of the coercivity is found to be well de- 


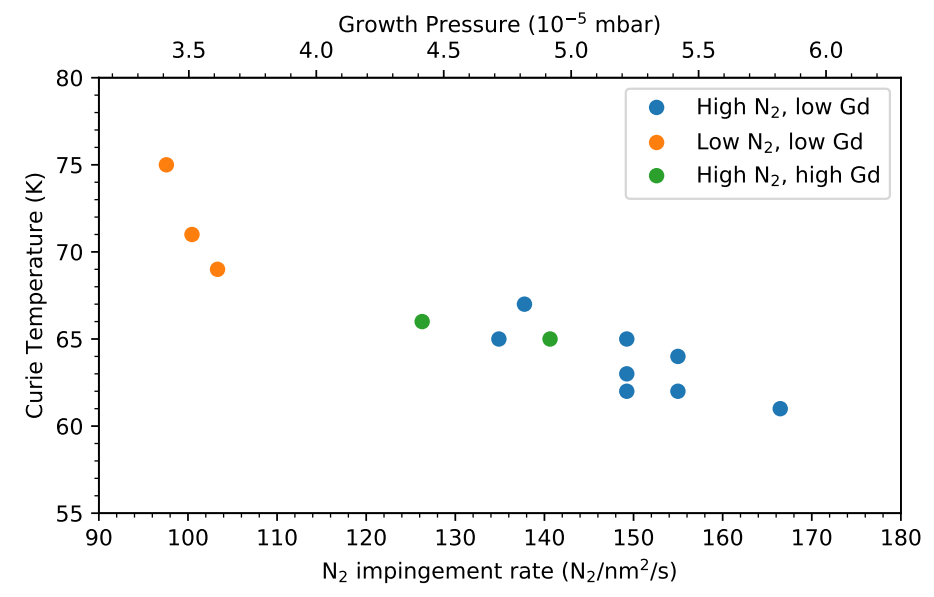

Figure 4.23: Curie temperature plotted against $\mathrm{N}_{2}$ impingement rate (growth pressure during deposition)

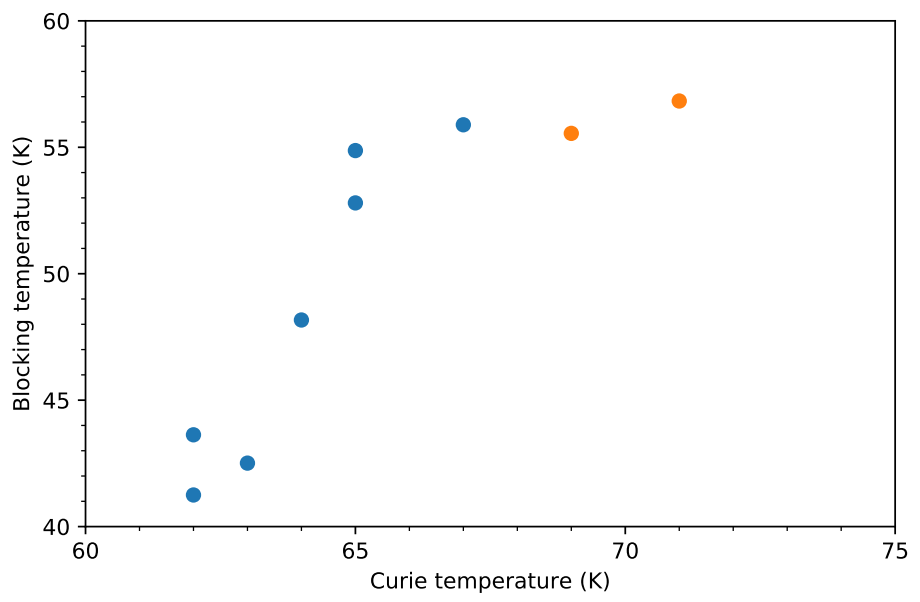

Figure 4.24: Comparison of the blocking temperature extracted from the coercivity temperature dependence with the Curie temperature extracted from the high temperature paramagnetic behaviour. 


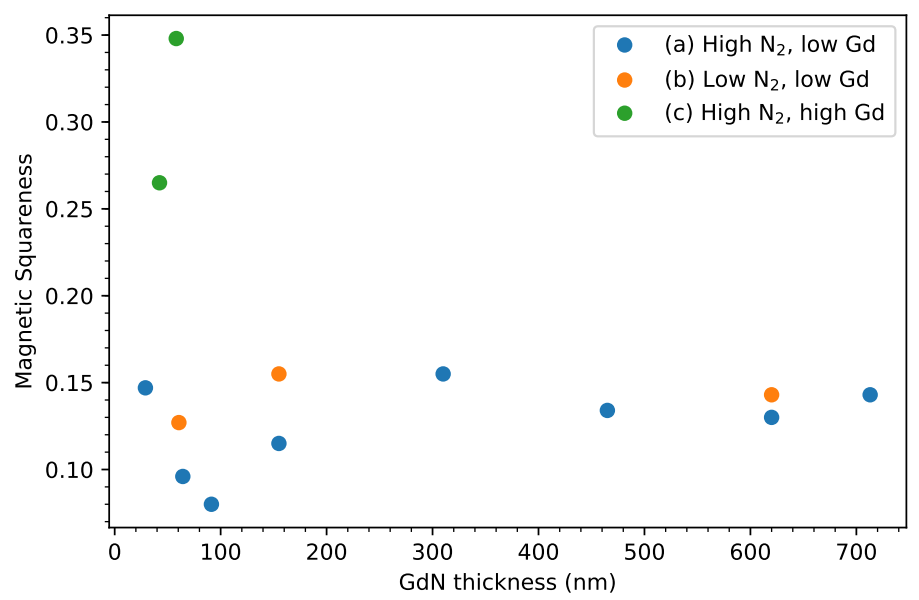

Figure 4.25: Magnetic squareness $M_{r} / M_{s}$ measured at $5 \mathrm{~K}$ plotted against GdN thickness

scribed by the equation

$$
H_{c}=H_{0}\left(1-{\frac{T}{T_{B}}}^{\frac{1}{2}}\right)
$$

where $H_{0}$ is the coercivity at $0 \mathrm{~K}$ and $T_{B}$ the blocking temperature. This relation is typical of nanoparticle systems [58, 59]. The blocking temperature extracted from this dependence is plotted against the Curie temperature for the same sample in Fig. 4.24 .

Another characterisation of the magnetic hysteresis is the "squareness", also known as the Stoner-Wohlfarth value, defined as $M_{r} / M_{s}$, the ratio between the remanent magnetisation and the saturation magnetisation [60]. In contrast to the Curie temperature, this value should be strongly dependent on the film microstructure. A value of 0.5 is expected for randomly oriented uniaxial grains; higher values are associated with texture formation while lower values are associated with larger magnetic particles and domain wall formation. For epitaxially grown ferromagnetic thin films, high values for this ratio $(>0.5)$ are often observed as one would expect from the epitaxial order; these would be ideal for applications such as memory devices incor- 


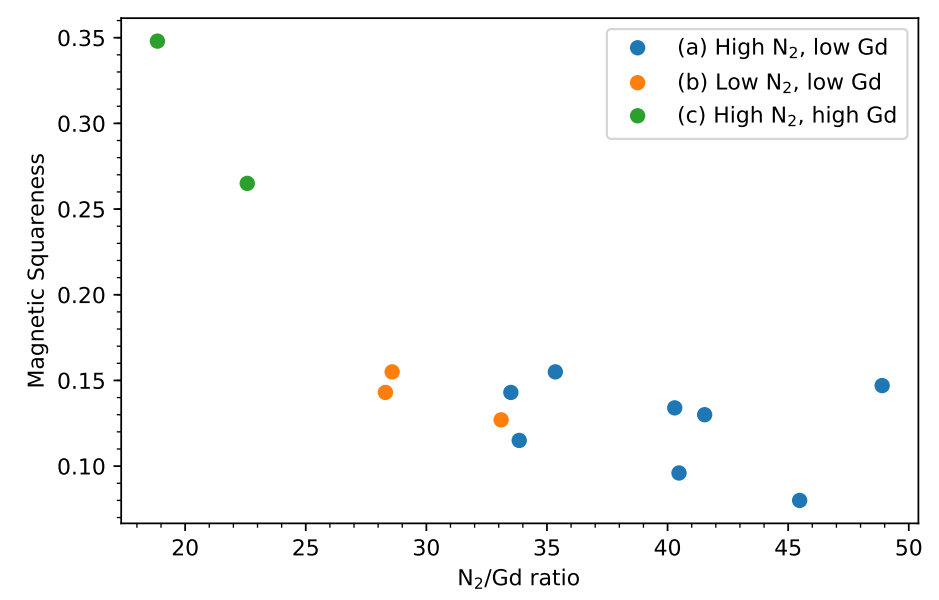

Figure 4.26: Magnetic squareness $M_{r} / M_{s}$ measured at $5 \mathrm{~K}$ as a function of the $\mathrm{N}_{2}$ : Gd ratio during deposition.

porating magnetic thin films which require high values for this ratio. For polycrystalline thin films, the value is typically lower than 0.5 .

The magnetic squareness for the GdN thin films measured at $5 \mathrm{~K}$ is plotted against the film thickness (Fig. 4.25) and $\mathrm{N}_{2}$ :Gd ratio (Fig. 4.26). For the majority of the samples, the magnetic squareness is 0.15 or smaller, but the two samples grown with high Gd flux have relatively high squareness values up to 0.35 .

\subsection{Discussion}

\subsubsection{Flux rate assumptions}

There are some issues with how the $\mathrm{Gd}$ and $\mathrm{N}_{2}$ flux rates are determined in Section 4.6 and the resulting flux ratio. The Gd flux is determined from the external thickness measurements/calibrations with the major assumption that the GdN film consists of dense, stoichiometric GdN. Given the nature of the room temperature deposition, the formation of voids is likely, although no quantitative measurement of the void density has been made. Metal- 
lic Gd clusters might also reduce the overall film density (bulk Gd density $7.90 \mathrm{~g} \mathrm{~cm}^{-3}$, GdN $\left.9.2 \mathrm{~g} \mathrm{~cm}^{-3}\right)$.

Unavoidable fluctuations in the electron beam evaporation of the $\mathrm{Gd}$ source mean that the flux rate will vary over the thickness of the film; the effect of this could be more significant for the thickest of the deposited GdN layers.

Similarly, the calculated $\mathrm{N}_{2}$ impingement rate likely deviates significantly from the true $\mathrm{N}_{2}$ flux incident on the substrate during deposition. The vacuum gauge that records the background pressure in the growth chamber is located at the top of the chamber, away from the substrate position, while the introduced $\mathrm{N}_{2}$ is partially directed towards the substrate. Understanding the complex pressure distribution involved with the mass flow inside the vacuum chamber is beyond the scope of this study, but a key point to note is that the background pressure is additionally intimately tied to the evaporation rate of the Gd source. As Gd evaporates and is deposited on the substrate, the walls of the chamber are also coated. This Gd acts as a "getter" with the residual gas (primarily consisting of $\mathrm{N}_{2}$ during growth) reacting with and adsorbing to the chamber walls. These factors mean that the simple impingement rate calculation is going to underestimate the true $\mathrm{N}_{2}$ flux on the substrate, and the relationship between the $\mathrm{N}_{2}$ flux rate and background pressure is not linear nor would it be constant, as it depends on other growth parameters beyond the set mass flow rate.

\subsection{2 $\mathrm{N}_{2}$ dissociative adsorption}

Many of the results presented in this chapter reveal the limitations in directly using the $\mathrm{N}_{2} / \mathrm{Gd}$ ratio. While a higher $\mathrm{N}_{2} / \mathrm{Gd}$ ratio should guarantee more nitrogen incorporation into the film, it does seem to have some drawbacks regarding the subsequent film microstructure such as possible incorporation of interstitial $\mathrm{N}$ or $\mathrm{N}_{2}$ in grain boundaries. Adsorbed $\mathrm{N}_{2}$ during deposition may inhibit diffusion of Gd adatoms. The catalytic dissociation effect allowing $\mathrm{N}$ to be incorporated from $\mathrm{N}_{2}$ also likely has diminishing returns with 
increased $\mathrm{N}_{2}$ flux on the surface, though this probably has a complicated relationship with the incident Gd flux as well. One future avenue for study may be exploring the effect of the growth pressure for varying Gd fluxes to optimise these effects.

\subsection{Conclusion}

The effect of the film thickness and other growth parameters such as deposition rate and $\mathrm{N}_{2}$ partial pressure during growth of polycrystalline GdN thin films has been investigated. The development of a preferential (111) orientation of GdN deposited on amorphous fused silica substrates has been understood as a result of evolutionary selection processes during film thickening.

The influence of the deposition parameters on the film microstructure and resulting electrical and magnetic properties was also investigated. A wide range of lattice constants were observed across samples, correlating with a huge range of film conductivities ranging from heavily doped semiconducting to highly insulating behaviour. The magnetic properties for all samples was consistent with previously reported magnetic results for GdN thin films, with Curie temperatures ranging between $60 \mathrm{~K}$ to $75 \mathrm{~K}$ and coercive fields at low temperatures between 200 Oe to 500 Oe as expected for disordered polycrystalline films. A few samples also exhibited saturation magnetisations close to the theoretical value of $7 \mu_{B} / \mathrm{Gd}^{3+}$, though this was not typical of most of the polycrystalline films. 


\section{Chapter 5}

\section{Epitaxial orientation transition in $\mathrm{SmN}$}

\subsection{Introduction}

Gaining access to epitaxial thin films has been essential in obtaining a better understanding of the fundamental properties of the REN series 7,24 , 25, 33 36, 61. In recent years, access to well-ordered $\mathrm{SmN}$ thin films has revealed the unusual and unique properties of SmN among the RENs; it is ferromagnetic at extremely low temperatures with a near zero magnetic moment due to the opposing contributions from the spin and orbital moments [9, 62]. In addition to this small moment, it is magnetically extremely hard, with the coercive field of $\mathrm{SmN}$ exceeding $8 \mathrm{~T}$ as the temperature approaches absolute zero 62].

At present, it is noticeable that most of the studies have been dedicated to fundamental magnetic properties of $\mathrm{SmN}$, although the discovery of a superconducting state of $\mathrm{SmN}$ was recently reported [63], possibly opening a means towards superconducting spintronics[64]. The epitaxial growth process picture is murky, with only a few publications reporting on it [25, 37. Our aim is to investigate the epitaxial growth, in particular the effect of the growth temperature and nitrogen precursor on the structural and electric 
properties.

$\mathrm{SmN}$ crystallises in the rock salt structure with a lattice parameter of $5.03 \AA$, slightly larger than that of GdN. Thin film growth of $\mathrm{SmN}$ can be achieved in the same manner as GdN, using molecular $\mathrm{N}_{2}$ as a nitrogen precursor which is catalytically broken down at the substrate by Sm metal or otherwise using thermally cracked $\mathrm{NH}_{3}$ grown at high temperatures. Compared to Gd, Sm has a lower melting point and higher vapour pressure, allowing Sm effusion cells to be operated at $550^{\circ} \mathrm{C}$ versus $\mathrm{Gd}$ at $1350{ }^{\circ} \mathrm{C}$. This leads to some interesting differences when it comes to the epitaxial integration with the III-V nitrides, in particular we will demonstrate a remarkable change in epitaxial growth orientation of epitaxial $\mathrm{SmN}$, controllable by the choice of growth temperature. The experiments confirming this observation and the investigation into the associated structural properties of epitaxial $\mathrm{SmN}$ are presented in this chapter.

Two publications related to the results in this chapter have been published:

[12]J. R. Chan, S. Vézian, J. Trodahl, M. A. Khalfioui, B. Damilano, and

F. Natali. "Temperature-Induced Four-Fold-on-Six-Fold Symmetric Heteroepitaxy, Rocksalt SmN on Hexagonal AlN". Crystal Growth \& Design 16.11 (2016), pp. 6454-6460

which focuses on the observation of the growth orientation crossover and the factors that lead to it, and

[13]J. R. Chan, M. Al Khalfioui, S. Vézian, J. Trodahl, B. Damilano, and F. Natali. "Effect of the growth temperature and nitrogen precursor on the structural and electrical transport properties of SmN thin films". MRS

Advances 2.3 (2017), pp. 165-171

which focuses on the effect of the growth temperature and choice of nitrogen precursor on the resulting structural and electrical properties of the SmN films. 
Additionally, a non-provisional patent concerning the results in this chapter has been filed to the United States Patent and Trademark Office

[15]F. Natali, S. Vézian, J. R. Chan, B. J. Ruck, and J. H. Trodahl. "Rare Earth Nitride Structure or Device and Fabrication Method". US Patent No. 15/607,596 (Filed 29 May 2017)

\subsection{Sample growth and measurement}

In 2013, a collaboration was initiated by my PhD supervisor, Dr. Franck Natali, with a research group at CNRS-CRHEA (Valbonne, France) to gain a better understanding of the material properties of RENs. This collaboration allowed access to significant experimental equipment dedicated to surface science studies. In particular, the MBE system connected under UHV to a STM, offering an excellent opportunity to investigate the relationship between growth conditions and surface morphology. A facility with such capabilities does not currently exist within NZ. I spent 1 month in June 2015 at CRHEA in order to use their facilities to study $\mathrm{SmN}$.

The samples analysed in this study were grown and measured at CNRSCRHEA primarily with the assistance from Drs. Stéphane Vézian, Benjamin Damilano and Mohamed Al Khalfioui. Growth was performed in an MBE system equipped with solid sources for $\mathrm{Gd}, \mathrm{Sm}, \mathrm{Mg}$ and $\mathrm{Ga}$, and pure $\mathrm{N}_{2}$ and $\mathrm{NH}_{3}$ as gaseous nitrogen precursors. SmN layers were grown on $100 \mathrm{~nm}$ AlN (0 0001 ) / Si (1 1 1) templates which were themselves grown by MBE. Details of the growth process for these AlN layers is presented in [65]. A full 2 inch template was cut into $1 \mathrm{~cm} \times 1 \mathrm{~cm}$ pieces which were outgassed and cleaned using a thermal annealing at $880^{\circ} \mathrm{C}$ under $\mathrm{NH}_{3}$ for 15 minutes to remove adsorbants. For the growth of the $\mathrm{SmN}$ layers, the Sm effusion cell was set to a temperature of $550{ }^{\circ} \mathrm{C}$ resulting in a maximum atomic flux of $1.3 \mathrm{~nm}^{-2} \mathrm{~s}^{-1}$ at the substrate or equivalently a maximum film growth rate of $150 \mathrm{~nm} \mathrm{~h}^{-1}$. For each sample, either $\mathrm{N}_{2}$ or $\mathrm{NH}_{3}$ was used as the nitrogen precursor with beam equivalent pressures $(\mathrm{BEP})$ of $2.7 \times 10^{-5}$ Torr and $1.9 \times 10^{-5}$ Torr re- 
spectively. The units of BEP system-specific, but here correspond to fluxes on the order of 100 times greater than the Sm flux, resulting in nitrogen-rich conditions. The templates were heated to facilitate epitaxial growth and set to fixed temperatures in the range $300{ }^{\circ} \mathrm{C}$ to $850^{\circ} \mathrm{C}$ as measured by optical infrared pyrometry. The emissivity of the optical pyrometer was calibrated by observing the well-known $(7 \times 7 \rightarrow 1 \times 1)$ Si (111) surface reconstruction via RHEED to get a transition temperature of $830^{\circ} \mathrm{C}$. For ex situ measurements, samples were then capped with $100 \mathrm{~nm}$ thick GaN layers. The effect of the growth temperature and the choice of nitrogen precursor on the growth kinetics and resulting quality of the $\mathrm{SmN}$ layer was studied through in-situ RHEED and STM, and ex-situ XRD and SEM.

The electrical transport properties of the $\mathrm{SmN}$ films were also measured and related to the structural properties. Hall effect and resistivity were measured at room temperature using a van der Pauw geometry.

\subsection{RHEED}

RHEED was used to monitor the nature and quality of the growth of layers being deposited on the substrate. As described in Chapter 3 , as a surface sensitive diffraction technique, RHEED provides information about whether deposited layers are epitaxial with the substrate, resulting in a discrete RHEED pattern of streaks or spots for example, or polycrystalline, with randomly oriented crystallites in the deposited layer resulting in diffraction rings. The streaks and spots that result from epitaxial growth are illustrative of the surface sensitivity; 3D diffraction from a single crystal should result in spots on the detector screen where the diffraction condition is satisfied, however when the diffraction surface is flat, these spots are often elongated into streaks due to the effective reduction of the crystal to a $2 \mathrm{D}$ lattice. In other words, a spotty RHEED pattern is indicative of a rough surface where $3 \mathrm{D}$ diffraction can occur while streaky RHEED patterns indicate a flat surface or growth front. 3D RHEED patterns calculated for varying orientations of single crys- 
tal $\mathrm{SmN}$, i.e. a cubic rock salt structure are shown in Fig. 5.1 to illustrate how crystal structure and orientation may be quickly identified from appropriately taken patterns. Squares and triangles are used to schematically represent respectively (001) and (111) growth orientations of a cubic rock salt crystal structure. By rotating the crystal such that the electron beam is directed along different crystal directions on each plane, different diffraction patterns are observed. The RHEED patterns resulting from the triangular (111) surface has been used to identify the rotational twinning that is observed for epitaxial growth of GdN (1 1 1) on GaN and AlN (0 0001$)$ substrates [34, 36] and is what we would also expect for epitaxial growth of $\mathrm{SmN}$ on AlN (0 0001$)$. However, we will demonstrate below that the diffraction patterns resulting from the square $\left(\begin{array}{ll}0 & 0\end{array}\right)$ orientation can also be obtained from growth of SmN on the same AlN (0 0001$)$ substrate.

RHEED patterns taken after the growth of successive layers of the $\mathrm{SmN}$ samples are shown in Fig. 5.2. The RHEED pattern of $\mathrm{SmN}$ is noted to form quickly after growth is started with a different lattice spacing than the AlN substrate, suggesting that the initial nucleation of $\mathrm{SmN}$ is not strained and subsequent growth is fully relaxed. The RHEED pattern of the GaN capping layer (Fig. 5.2 d) indicates rough growth as well; the capping layer quality is strongly dependent on the growth process and resulting quality of the SmN layer below. For the same growth temperature, there is a clear difference in crystalline quality of $\mathrm{SmN}$ grown with either $\mathrm{N}_{2}$ (Fig. 5.2b) or $\mathrm{NH}_{3}$ (Fig. 5.2. ). The narrow peaks in the horizontal direction present in Fig. 5.2b compared to c are indicative of a larger coherence length, while elongation in the vertical direction ("streakiness") corresponds to a smoother surface for growth with $\mathrm{N}_{2}$ at the shown temperature. For samples grown at the highest growth temperatures tested, a previously unobserved RHEED pattern emerges (Fig. 5.3b) which we now turn our focus to.

Fig. 5.1 showing RHEED patterns resulting from single crystal $\mathrm{SmN}$ in different orientations helps in understanding the observed SmN RHEED patterns. A close analysis of the final SmN RHEED patterns are shown in 

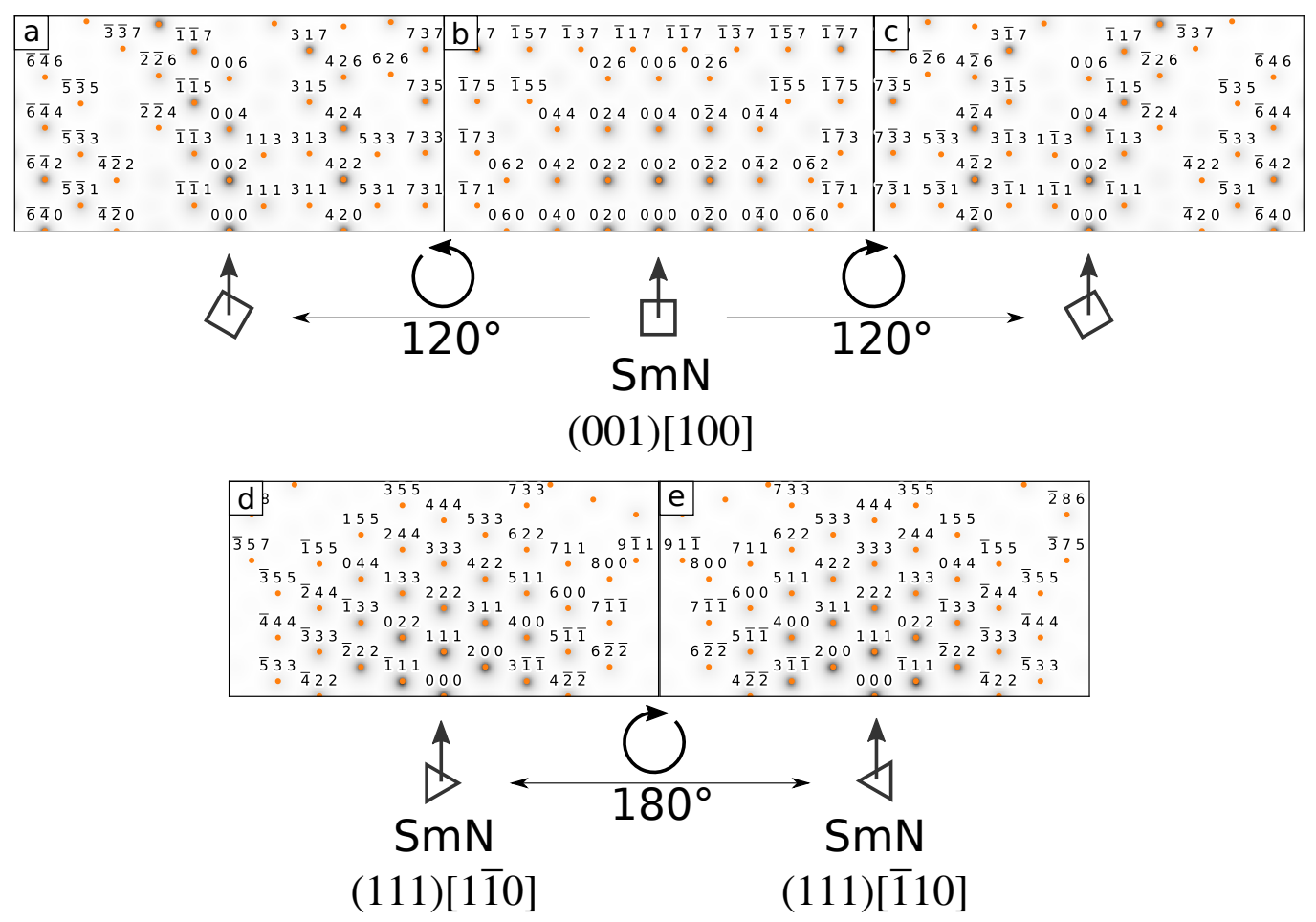

Figure 5.1: RHEED diffraction maxima (orange spots) for single crystal SmN with the (0 01 ) planes $(\mathrm{a}-\mathrm{c})$ and (111) planes (d, e) perpendicular to the growth axis. The electron beam is directed along the $\left[\begin{array}{ll}1 & 0\end{array}\right]$ direction in (b), and along the $\left[\begin{array}{ll}1 & 1\end{array}\right]$ and $\left[\begin{array}{ll}1 & 10\end{array}\right]$ directions in (d) and (e). (a) and (c) highlight the non-regularity of the resulting patterns when the electron beam is not parallel to an integer miller direction; the patterns shown result from $120^{\circ}$ azimuthal rotations of (b) anticlockwise and clockwise respectively. Note that these rotations are equivalent to $30^{\circ}$ clockwise and anticlockwise rotations with the fourfold symmetry of the (001) plane. 

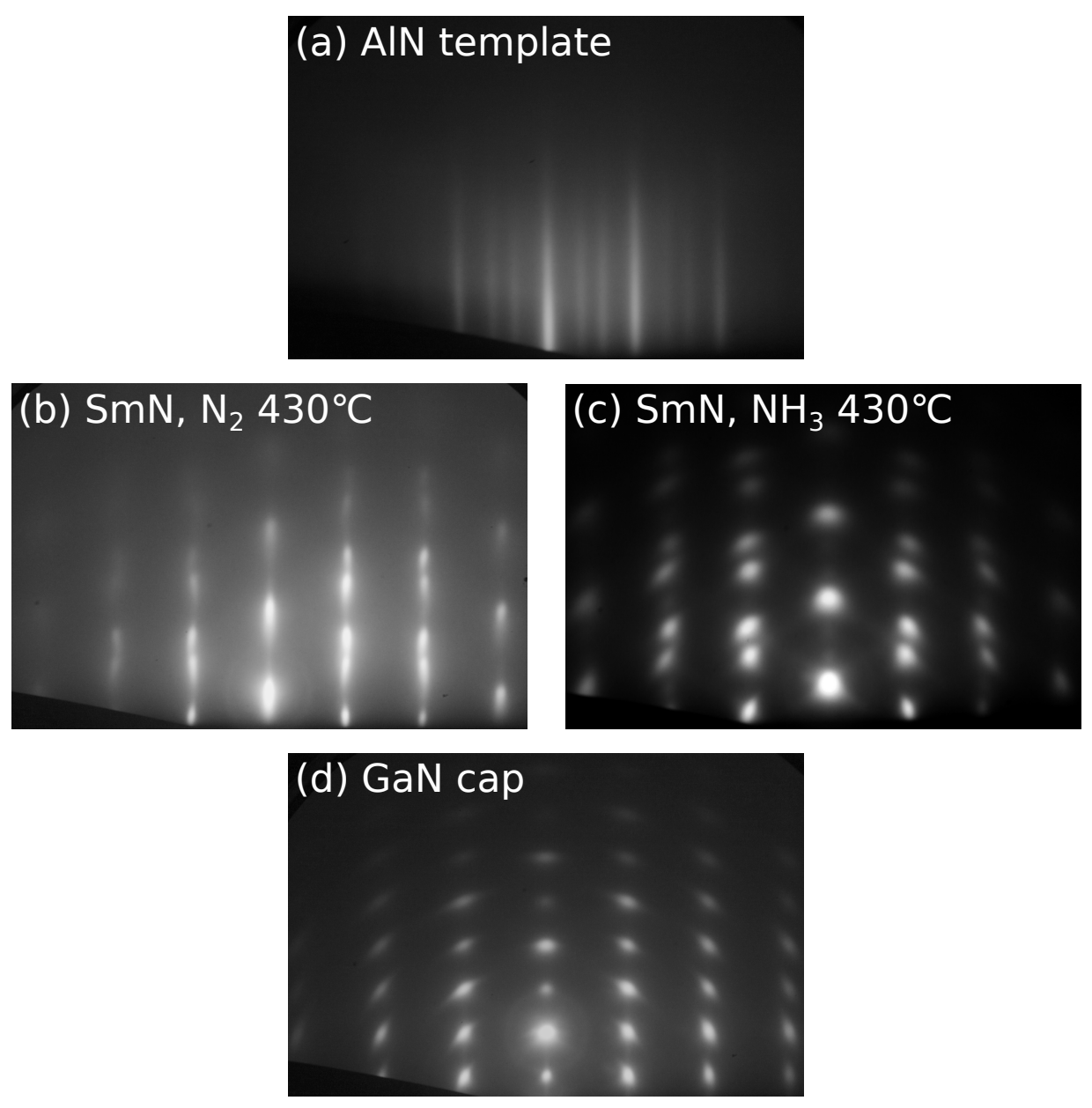

Figure 5.2: Typical RHEED patterns taken at different stages of growth of epitaxial SmN thin films. (a) Streaky RHEED pattern along

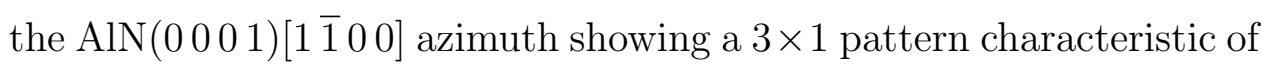
a $\sqrt{3} \times \sqrt{3}$-R30 ${ }^{\circ}$ reconstruction seen in Al-polar AlN [66]. (b, c) Spotty RHEED patterns along the same azimuth as in (a) of $\mathrm{SmN}$ grown at $430{ }^{\circ} \mathrm{C}$ with (b) $\mathrm{N}_{2}$ and (c) $\mathrm{NH}_{3}$. (d) Typical spotty RHEED pattern for $\mathrm{GaN}$ grown on $\mathrm{SmN}$ as before. 

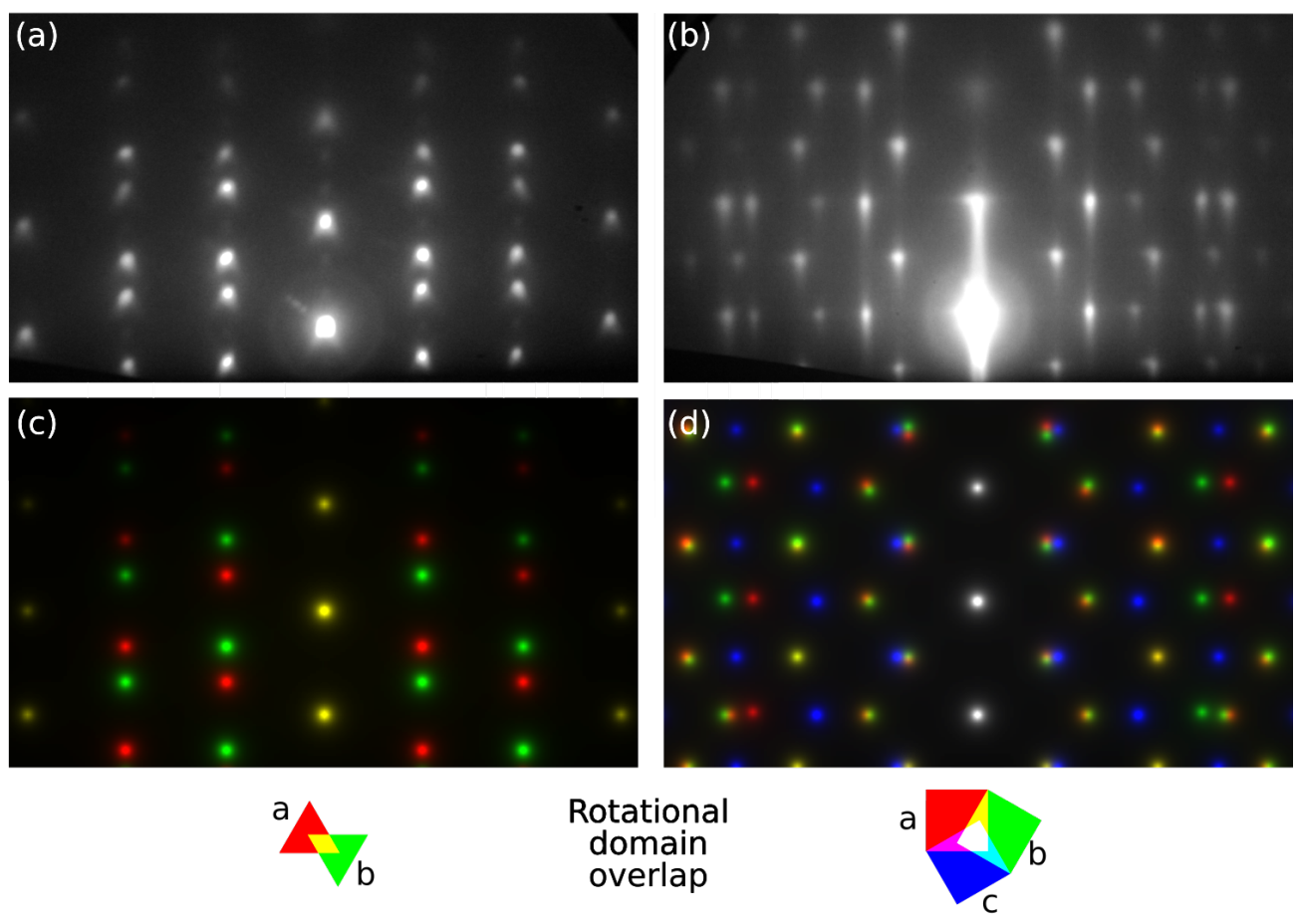

Figure 5.3: RHEED images taken for $100 \mathrm{~nm}$ thick samples of $\mathrm{SmN}$ grown at (a) $700{ }^{\circ} \mathrm{C}$ and (b) $800{ }^{\circ} \mathrm{C}$ using $\mathrm{NH}_{3}$ as the nitrogen precursor along the $\langle 11 \overline{2} 0\rangle$ azimuth of AlN. Simulations of the respective RHEED patterns are produced by considering (c) (111)-oriented SmN with twin domains rotated by $180^{\circ}$ with respect to each other, along the $\langle 1 \overline{1} 0\rangle \mathrm{SmN}$ azimuth, and (d) (0 01 )-oriented $\mathrm{SmN}$ with triplet domains rotated by $120^{\circ}$ with respect to each other, along the $\langle 110\rangle \mathrm{SmN}$ azimuth. Contributions from each $\mathrm{SmN}$ domain are separated out into red, green and blue components. 
Fig. $5.3 \mathrm{a}$ and b. The final SmN pattern in Fig. $5.3 \mathrm{a}$ is a result of twin domains rotated $180^{\circ}$ with respect to each other (Fig. 5.3 ); this result has been similarly observed for growth of GdN on GaN 34] and AlN 24] (0001) surfaces. Unlike the epitaxial growth of GdN, however, the emergence of a distinct RHEED pattern is observed at elevated growth temperatures (Fig. 5.3b). The onset of this shift differs for the two nitrogen precursors, $600{ }^{\circ} \mathrm{C}$ using $\mathrm{N}_{2}$ and above $750^{\circ} \mathrm{C}$ using $\mathrm{NH}_{3}$. This new RHEED pattern can be reproduced by considering a triplet of rotational domains of ( 001$)$-oriented $\mathrm{SmN}$ rotated $120^{\circ}$ with respect to one another (Fig. $5.3 \mathrm{~d}$ ). It is worth noting that the RHEED patterns resulting from the (001)-oriented growth consist of elongated diffraction spots indicating a smoother surface; this is corroborated by in situ results presented later.

\subsection{XRD}

XRD measurements were performed on all SmN samples to complement the surface crystallinity RHEED measurements with bulk-sensitive diffraction in both symmetric and asymmetric geometries. Symmetric $2 \theta-\omega$ scans are shown in Fig. 5.4 and illustrate the cross-over in growth orientation of $\mathrm{SmN}$ as a function of growth temperature. The peaks in the $2 \theta-\omega$ gives information along the direction perpendicular to the substrate, i.e. the growth direction. For moderate growth temperatures (up to $500{ }^{\circ} \mathrm{C}$ with $\mathrm{N}_{2}$ and up to $700{ }^{\circ} \mathrm{C}$ with $\mathrm{NH}_{3}$ ) the only visible $\mathrm{SmN}$ peak is the 111 , i.e. the growth orientation of $\mathrm{SmN}$ is (111) on the AlN (0001) substrate, similar to the observed growth orientation of other epitaxial RENs on (0001) substrates

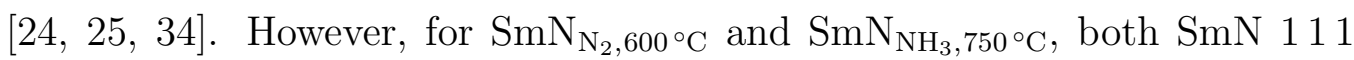
and 002 reflections are seen and in the $2 \theta-\omega$ scan of $\mathrm{SmN}_{\mathrm{NH}_{3}, 800}{ }^{\circ} \mathrm{C}$, the 111 reflection disappears completely and only the $\mathrm{SmN} 002$ peak is seen. This is consistent with the observations from RHEED where the pattern ob-

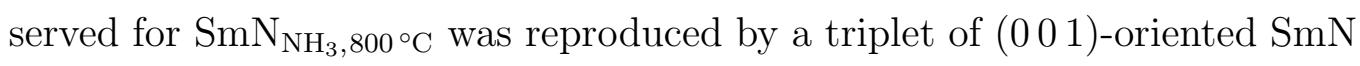
rotational domains. 


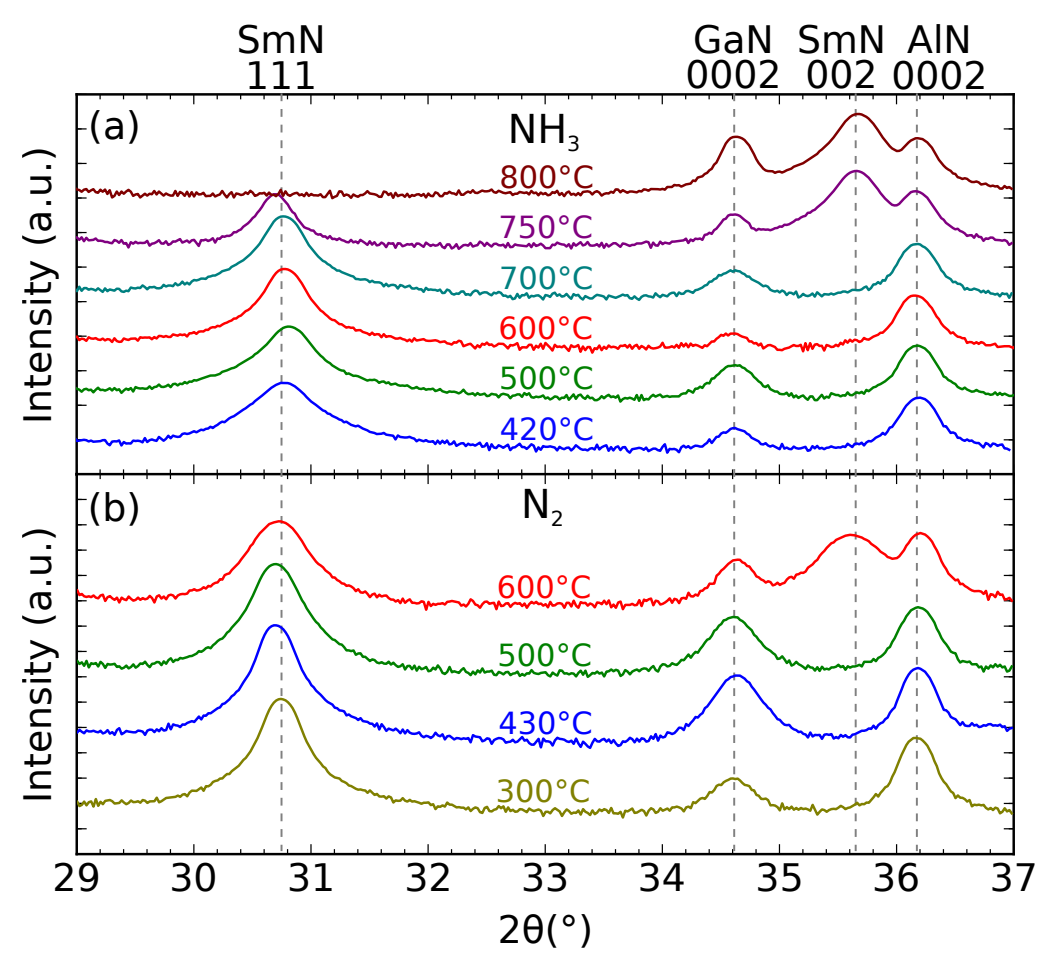

Figure 5.4: XRD $2 \theta-\omega$ scans for varying growth temperatures of SmN using (a) $\mathrm{NH}_{3}$ and (b) $\mathrm{N}_{2}$ as the nitrogen precursors. The $\mathrm{SmN}$ layer thickness is typically about $100 \mathrm{~nm}$. At $600{ }^{\circ} \mathrm{C}$ using $\mathrm{N}_{2}$ and $750{ }^{\circ} \mathrm{C}$ using $\mathrm{NH}_{3}$, mixed (111)- and (001)-oriented growth of SmN occurs; below these temperatures the growth is fully (111)-oriented. At the highest growth temperature of $800{ }^{\circ} \mathrm{C}$, only the $\mathrm{SmN} 002$ peak is seen indicating a transition to fully (0 01$)$-oriented growth. 


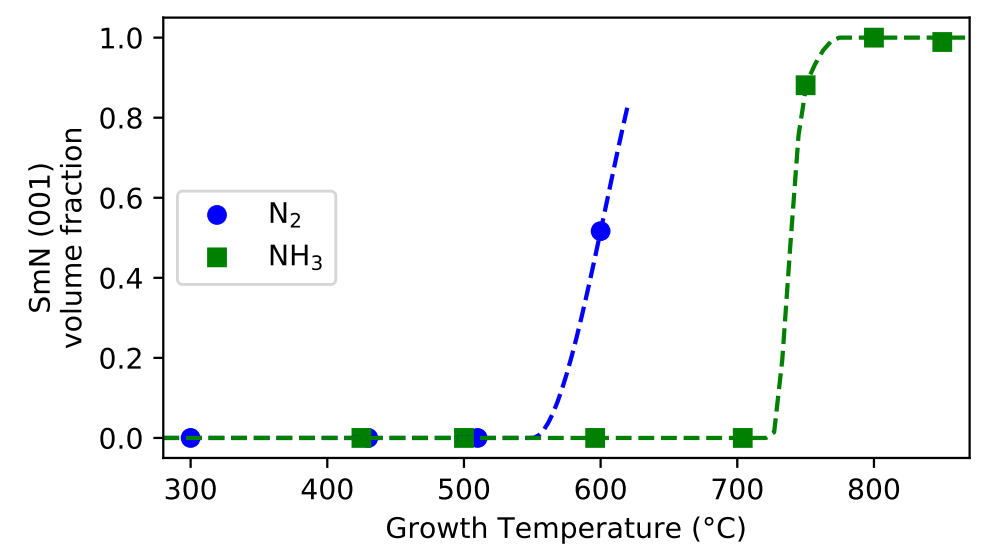

Figure 5.5: Volume fraction of $\mathrm{SmN}\left(\begin{array}{lll}0 & 0 & 1\end{array}\right)$ crystallites oriented parallel to the substrate as a function of growth temperature for $\mathrm{N}_{2}$ and $\mathrm{NH}_{3}$ nitrogen precursors calculated using 5.1. The dashed lines are a guide for the eye.

The volume fraction $V_{f}$ of $\mathrm{SmN}\left(\begin{array}{lll}0 & 0 & 1\end{array}\right)$ crystallites oriented parallel to the film surface can be estimated using the equation

$$
V_{f}=\frac{I_{(002)} / S_{(002)}}{I_{(002)} / S_{(002)}+I_{(111)} / S_{(111)}}
$$

where $I_{(002)}$ and $I_{(111)}$ are the integrated areas of the 002 and 111 reflections divided by the structure factors $S_{(002)}$ and $S_{(111)}$ which are defined such that a uniformly randomly oriented (powder) sample results in an equal volume fraction for each phase considered. A theoretical calculation of this structure factor gives $S_{(002)}=0.7347$ and $S_{(111)}=1$. The change in volume fraction as a function of temperature for both nitrogen precursors is give in Fig. 5.5 .

The epitaxial relationship between the $\mathrm{SmN}$ layers in both orientations and the AlN substrate was further investigated by performing asymmetric $\phi$ scans (Fig. 5.6 and b). A representative scan for a (111)-oriented $\mathrm{SmN}$ shows that the $\mathrm{SmN} 002$ reflection has 6 peaks spaced $60^{\circ}$ equally apart on top of the 6 peaks of the AlN $10 \overline{1} 2$ reflection. This is characteristic of twin domains (as previously observed [24, 25, 34]) as the 002 reflection 


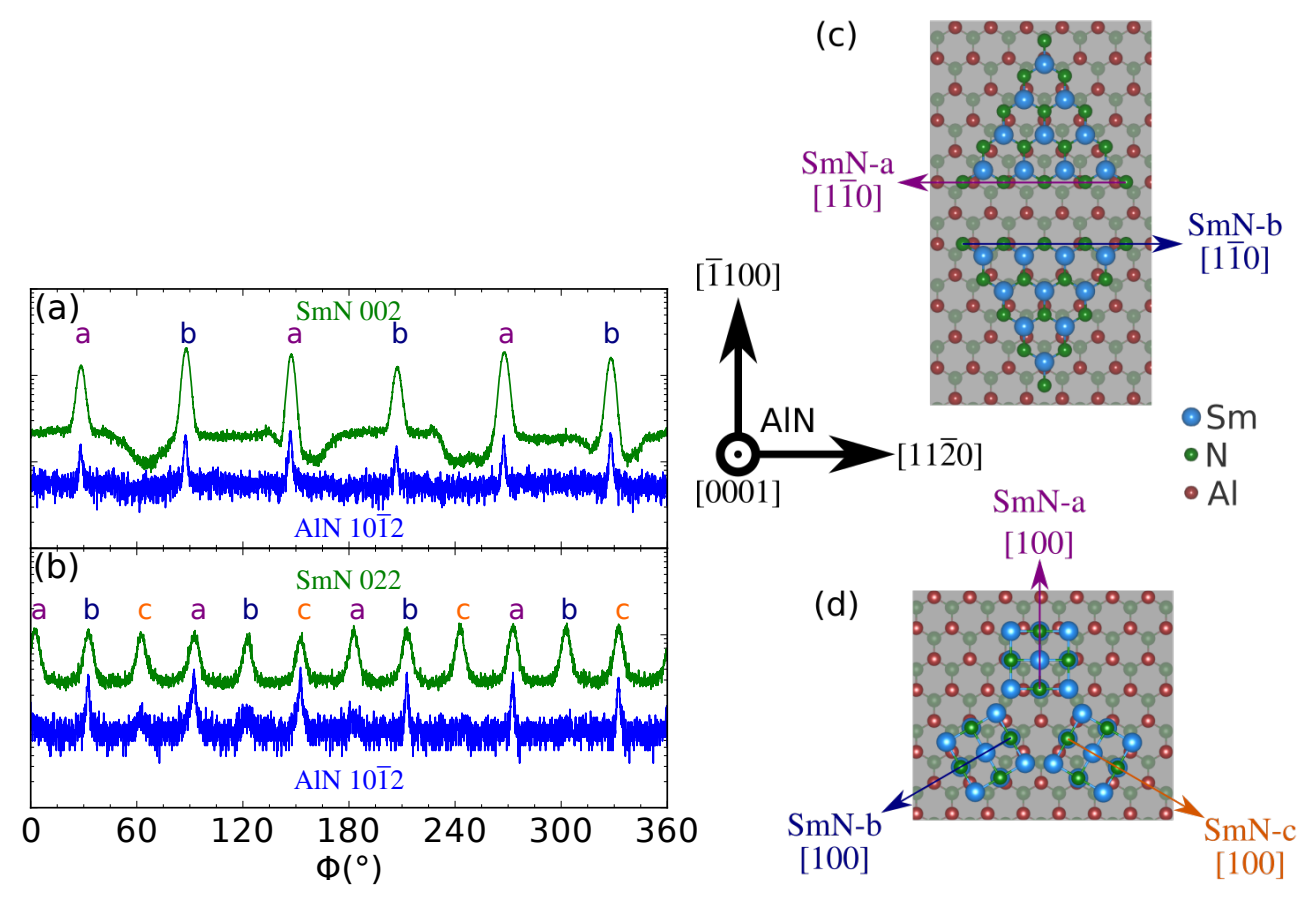

Figure 5.6: $\mathrm{XRD} \phi$ scans of $\mathrm{SmN}$ samples grown using $\mathrm{NH}_{3}$ at (a) $700{ }^{\circ} \mathrm{C}$ and (b) $800^{\circ} \mathrm{C}$. The 6 -fold symmetry of the AlN $10 \overline{1} 2$ reflection is compared to (a) the 6-fold symmetry from two rotational domains (labelled a and b) of (111)-oriented $\mathrm{SmN}$ and (b) the 12-fold symmetry from three rotational domains (labelled a, b, and c) of ( 001$)$-oriented SmN. Schematic atomic models of the respective inferred in-plane epitaxial relationships are shown in (c) and (d). 
of a (111)-oriented single crystal should only produce 3 peaks, i.e. 3-fold symmetry. The epitaxial relationship in this case is (Fig. 5.6.),

$$
\begin{aligned}
& \operatorname{SmN}\left(\begin{array}{lll}
1 & 1)
\end{array}\right) \operatorname{AlN}\left(\begin{array}{llll}
0 & 0 & 0 & 1
\end{array}\right) \\
& \operatorname{SmN}(111)\langle 1 \overline{1} 0\rangle \| \operatorname{AlN}(0001)\langle 11 \overline{2} 0\rangle
\end{aligned}
$$

For $(001)$-oriented $\mathrm{SmN}\left(\mathrm{SmN}_{\mathrm{NH}_{3}, 800}{ }^{\circ} \mathrm{C}\right)$, the $\phi$ scan of the $\mathrm{SmN} 022$ reflection produces 12 peaks spaced $30^{\circ}$ apart on top of the AlN $10 \overline{1} 2$. The 022 reflection of a (001)-oriented single crystal should exhibit 4-fold symmetry, i.e. this set of 12 peaks is indicative of triplet rotational domains rotated by $120^{\circ}$ relative to each other. In this case, the epitaxial relationship is (Fig. 5.6 d)

$$
\begin{aligned}
& \operatorname{SmN}\left(\begin{array}{lll}
0 & 0 & 1)
\end{array}\right) \operatorname{AlN}\left(\begin{array}{llll}
0 & 0 & 0 & 1
\end{array}\right)
\end{aligned}
$$

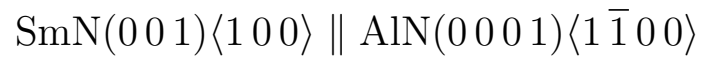

The schematic atomic representation Fig. 5.6c shows two triangle rotational domains rotated by $180^{\circ}$ relative to each other while Fig. 5.6 d shows three square domains rotated by $120^{\circ}$ relative to each other.

A qualitative analysis of the structural quality is obtained by looking at the line widths of the peaks in the $2 \theta-\omega$ scans as well as of the rocking curves of the respective SmN peaks. Broadening of the line widths in the $2 \theta-\omega$ scans are due to size and strain effects in the relevant crystallites; although the collected data do not allow for a complete separation of these components. Smaller linewidths are simply interpreted as the combination of longer out-of-plane coherence lengths and/or smaller strain distributions. The $2 \theta-\omega$ peak widths are plotted in Fig. 5.7. Most line widths of the SmN 111 and 002 reflections lie between $0.20^{\circ}$ to $0.25^{\circ}$ but notably outside this range are two samples $\mathrm{SmN}_{\mathrm{NH}_{3}, 425^{\circ} \mathrm{C}}\left(0.37^{\circ}\right)$ and $\operatorname{SmN}_{\mathrm{N}_{2}, 425^{\circ} \mathrm{C}}\left(0.16^{\circ}\right)$. An even clearer difference between the nitrogen precursors as a function of growth temperature is seen in the FWHM of the rocking curves of the $\mathrm{SmN}$ (111) and (002) peaks (Fig. 5.8). Rocking curves measure the degree of 


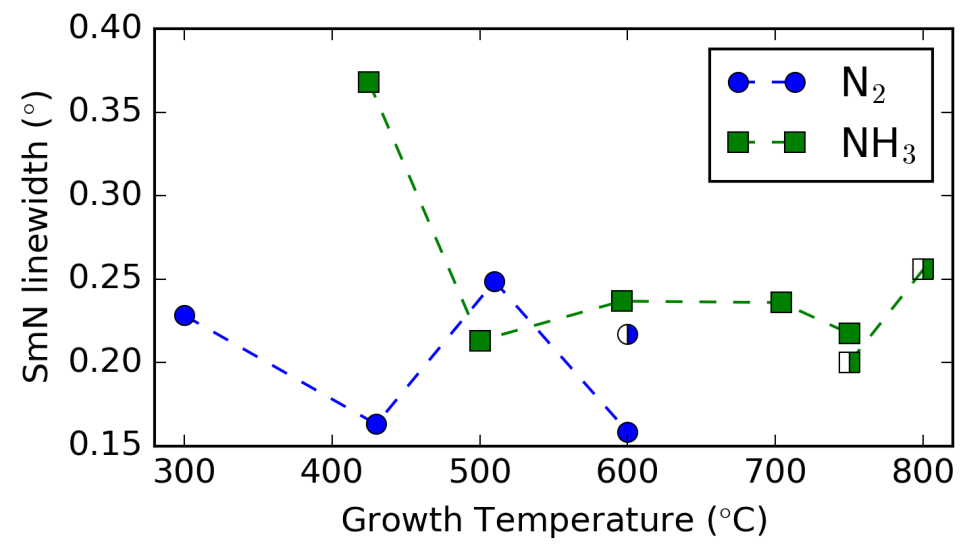

Figure 5.7: Linewidth of the $\mathrm{SmN}$ peaks in the $2 \theta-\omega$ scans from Fig. 5.4 as a function of growth temperature and nitrogen precursor. Fully filled markers correspond to the SmN 111 peak while half-filled markers are from the SmN 002 peak.

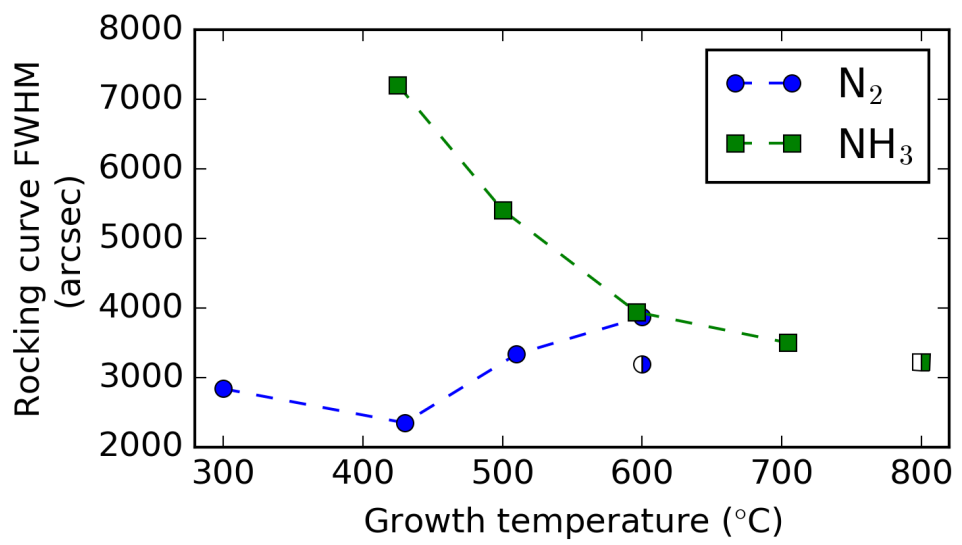

Figure 5.8: XRD out-of-plane rocking curve full width at halfmaximum (FWHM) as a function of the growth temperature and nitrogen precursor. Fully filled markers represent the FWHM of the $\mathrm{SmN}$ 111 rocking curve while the half-filled markers are of the $\mathrm{SmN} 002$ rocking curve. 

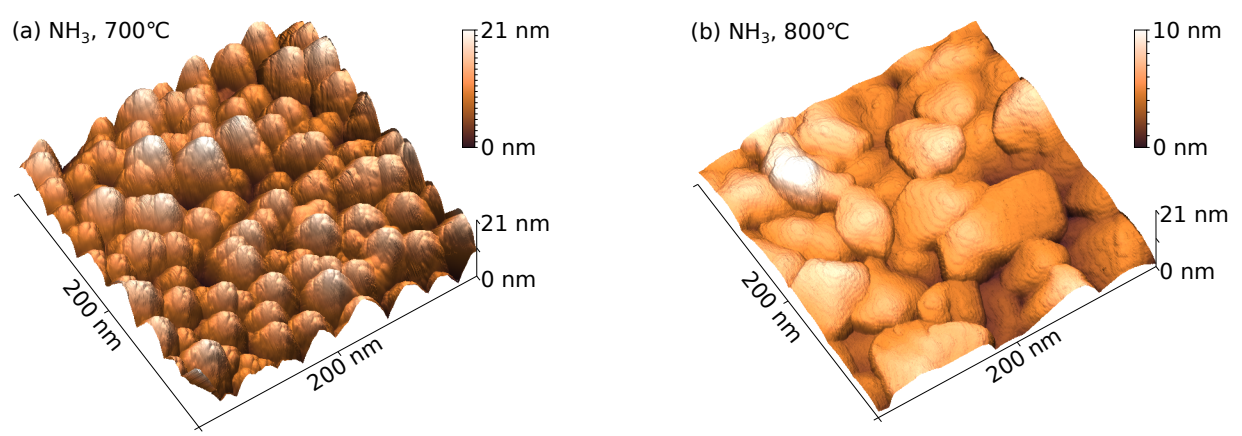

Figure 5.9: $200 \mathrm{~nm} \times 200 \mathrm{~nm}$ STM images of $\mathrm{SmN}$ samples grown using $\mathrm{NH}_{3}$ at (a) $700{ }^{\circ} \mathrm{C}$ and (b) $800{ }^{\circ} \mathrm{C}$ after deposition of $100 \mathrm{~nm}$. Acquisition parameters were $1 \mathrm{nA}$, and $1.5 \mathrm{~V}$

mosaicity, with a smaller FWHM indicating a smaller distribution of crystallites away from the perpendicular. The FWHM is minimised with the $\mathrm{N}_{2}$ precursor at a growth temperature around 430 $(\mathrm{SmN} 111 \mathrm{FWHM}=2350$ arcsecond) whereas the FWHM monotonically decreases as the growth temperature increases when using $\mathrm{NH}_{3}$ to a minimum at $700{ }^{\circ} \mathrm{C}$ just before the onset of the growth orientation transition ( $\mathrm{SmN} 111=3500$ arcsecond).

\section{$5.5 \mathrm{STM}$}

The change in surface morphology over the growth orientation transition was

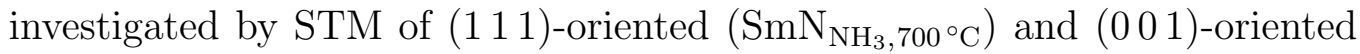
$\left(\mathrm{SmN}_{\mathrm{NH}_{3}, 800}{ }^{\circ} \mathrm{C}\right)$ samples. This is the first time that the surface of $\mathrm{SmN}$ has been directly imaged; preliminary results on the surface of epitaxial GdN have been obtained but only on (111)-oriented $\mathrm{GdN}[36]$. $100 \mathrm{~nm}$ thick layers of $\mathrm{SmN}$ were grown using the aforementioned conditions and transferred in situ to an STM system connected under vacuum to the growth system. $200 \mathrm{~nm} \times$ $200 \mathrm{~nm}$ STM images of the final SmN surface are shown in Fig. 5.9. There is a clear decrease in the root-mean-square surface roughness from $3.20 \mathrm{~nm}$ for the (111)-oriented SmN to $1.46 \mathrm{~nm}$ for the (0 01 )-oriented SmN surface over the imaged area. This is also accompanied by an increase in the average lateral 


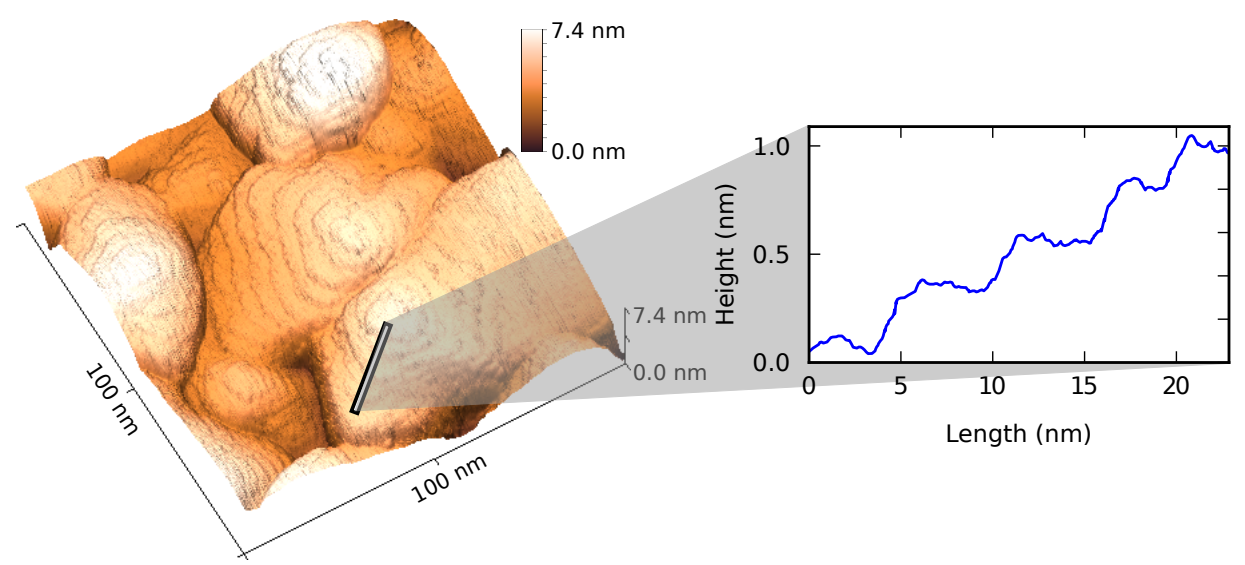

Figure 5.10: Zoomed image of 5.9b showing atomic steps on the crystallite grains corresponding to half of the lattice constant of $\mathrm{SmN}$ $(2.52 \AA)$

grain size from $(19 \pm 2) \mathrm{nm}$ to $(33 \pm 2) \mathrm{nm}$. This is rather notable that even through there are 3 rotational domains for the $\left(\begin{array}{lll}0 & 0 & 1\end{array}\right)$-oriented growth, there is a decrease in the surface roughness and increase in lateral grain size. It is worth pointing out that steps corresponding to half of the lattice constant of $\mathrm{SmN}(2.52 \AA)$, i.e. one atomic monolayer are visible on the STM image of (0 01 )-oriented SmN (Fig. 5.10).

\subsection{Electrical transport}

Electrical transport measurements were carried out using a standard four terminal measurement in the van der Pauw geometry using pressed indium contacts on top of the GaN capping layer. Room temperature measurements of the resistivity, carrier concentration and electron mobility are shown in Fig. 5.11. The resistivity and carrier concentrations of the $\mathrm{SmN}$ films are on the order of $1 \mathrm{~m} \Omega \mathrm{cm}$ and $10^{21} \mathrm{~cm}^{-3}$; similar resistivities and carrier concentrations have been widely reported for nominally undoped epitaxial REN thin films $34,36,61$ and are normally attributed to nitrogen vacancy formation. The low formation energy of nitrogen vacancies, which can dope the 

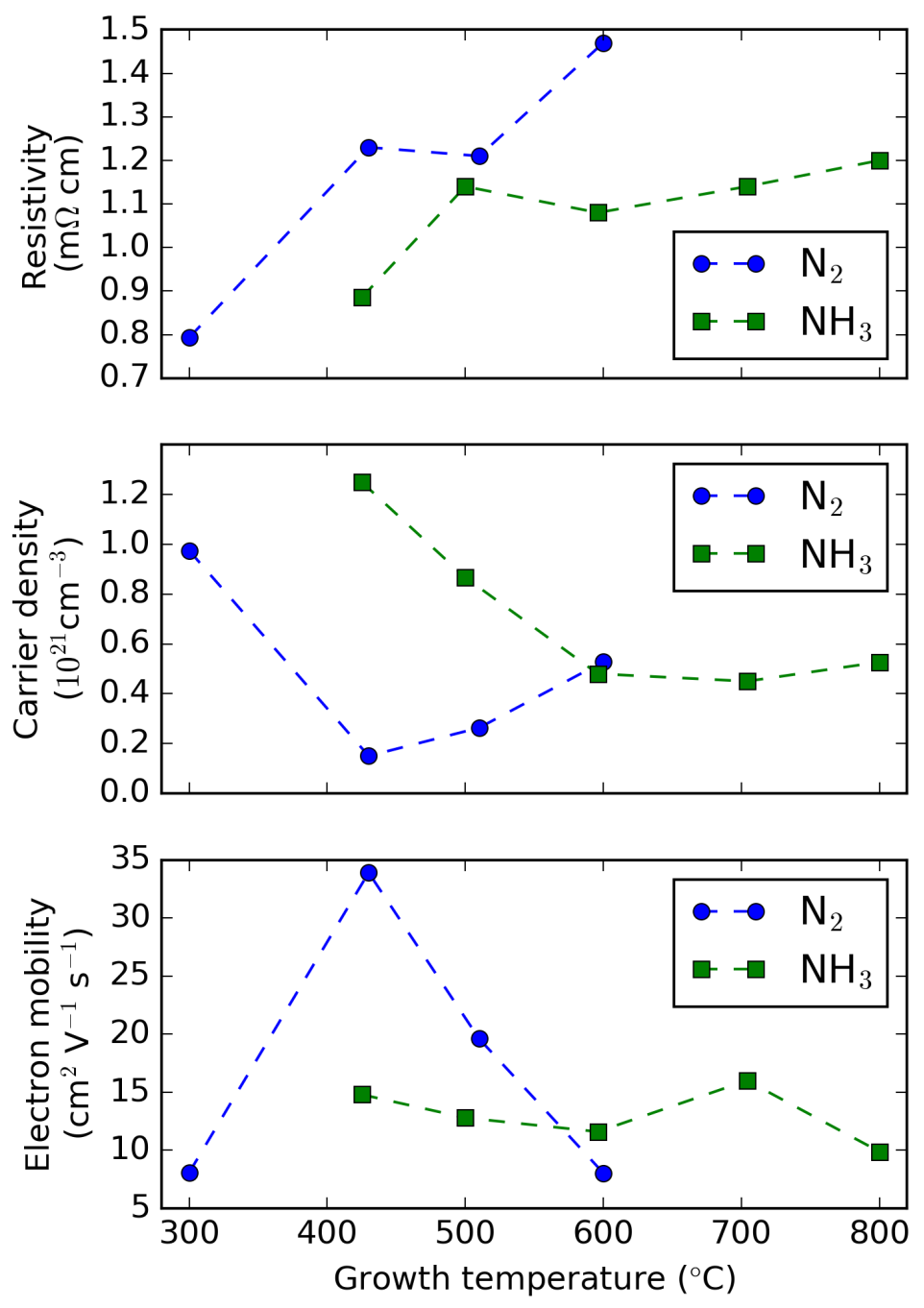

Figure 5.11: Electrical transport [(a) resistivity, (b) carrier density, (c) electron mobility] of $\mathrm{SmN}$ measured at room temperature as a function of growth temperature and nitrogen precursor. 
film with up to three electrons each, ensures that the vacancy concentration be on the order of $1 \%$ in epitaxial films.

For both nitrogen precursors, the resistivity tends to increase with increasing growth temperature of $\mathrm{SmN}$ though the increase is less than a factor of two. Carrier concentration shows a much larger variation which correlates well with the crystalline quality as measured by XRD (Fig. 5.8); $\mathrm{SmN}_{\mathrm{N}_{2}, 425^{\circ} \mathrm{C}}$ which had the smallest rocking curve FWHM measured of 2350" also had the lowest carrier concentration and highest electron mobility measured of $1.5 \times 10^{20} \mathrm{~cm}^{-3}$ and $33.9 \mathrm{~cm}^{2} \mathrm{~V}^{-1} \mathrm{~s}^{-1}$ respectively.

\subsection{Discussion}

The observation of a controllable growth-orientation of $\mathrm{SmN}$ on a $\mathrm{AlN}$ (0001) surface is clearly identified by RHEED and XRD measurements. Systems that exhibit epitaxial growth orientation control through growth parameters are rare; one example is the epitaxial growth of $\mathrm{Pt}$ on $\mathrm{MgO}$ 67. The other unusual aspect of the $\mathrm{SmN} / \mathrm{AlN}$ system is the resultant "cube-onhexagon" geometry; symmetry matching of the close-packed planes heavily favours (111)-oriented growth. Nevertheless, there are a few examples of $\left\{\begin{array}{lll}0 & 0 & 1\end{array}\right\}$-oriented growth on substrates with 3 - or 6 -fold symmetry 68 70]. We continue with a discussion of the influencing factors that allow the transition to occur, including symmetries of rotational domains in heteroepitaxy, the precise role of growth temperature and factors specific to the growth of $\mathrm{SmN}$ and the role of the nitrogen precursor.

\subsubsection{Epitaxial domains and growth orientation}

The formation of (often unwanted) rotational domains in heteroepitaxial systems where there is a mismatch in the crystal symmetries of the substrate and epilayer is a natural consequence of the number of equivalent ways which the epilayer material might crystallise on the substrate. A mathematical formal- 


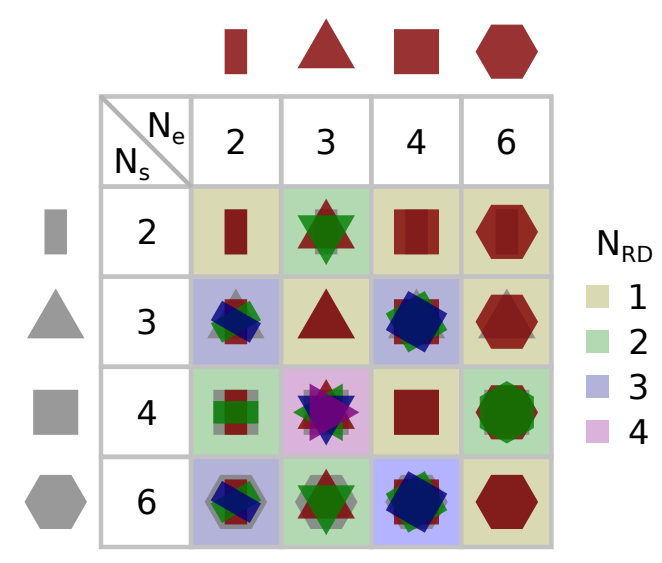

Figure 5.12: A schematic representation of the minimum number of rotational domains formed due to the number of equivalent ways an epilayer with rotational symmetry $N_{e}$ can be placed on a substrate with rotational symmetry $N_{s}$.

isation has been given by Grundmann [71] to predict the minimum number of rotational domains that might be expected given the $2 \mathrm{D}$ point symmetries of the crystalline materials being considered:

$$
N_{\mathrm{RD}}(n, m)=\frac{\operatorname{lcm}(n, m)}{m}
$$

where $n$ and $m$ denote the $C_{n}$ and $C_{m}$ rotational symmetries of the substrate and epilayer, and $\operatorname{lcm}(n, m)$ the least common multiple of $n$ and $m$. This is shown graphically for $n, m \in\{2,3,4,6\}$ in Fig. 5.12. It is desirable to choose substrates that have matching symmetry to the epilayer material in question, but it is not uncommon to exploit the (111) face of cubic materials $(n=6)$ to explore growth on hexagonal substrates. This has been done for RENs on III-nitrides [34] which take the hexagonal wurtzite structure $(m=3)$, as the number of suitable cubic substrates for RENs is otherwise limited $[7]$. The consequence as noted above is the formation of rotational domains $\left(N_{\mathrm{RD}}(6,3)=2\right)$, and the point symmetry of the substrate would need to be broken somehow to suppress or even eliminate the rotational domain, e.g. by using a miscut or rough substrate 72 . 
The growth orientation transition exhibited by $\mathrm{SmN}$ on AlN introduces a new case of "cube-on-hexagon" epitaxy where $m=4$ and thus the expected number of rotational domains becomes $N_{\mathrm{RD}}(6,4)=3$. Heteroepitaxial systems reported in the literature exhibiting similar (001)-oriented

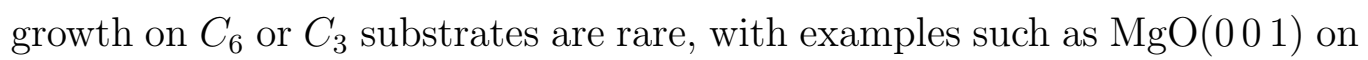
$\mathrm{ZnO}\left(\begin{array}{lll}0 & 0 & 1\end{array}\right)$ 68, $\mathrm{SrB}_{6}$ on ultrasmooth $\left(\begin{array}{llll}0 & 0 & 1\end{array}\right) \alpha-\mathrm{Al}_{2} \mathrm{O}_{3}\left(\begin{array}{llll}0 & 0 & 1\end{array}\right)$ 69 and STO on $\mathrm{GaN}$ [3]. However, not much attention is given to trying to understand the fundamental mechanisms driving this orientation matching or the growth orientation transitions.

Beyond simple symmetry conditions, no complete theories exist to predict the epitaxial growth orientation for dissimilar crystalline materials with significant lattice mismatch [74], and indeed, more than one orientation is possible as illustrated by this system. Nevertheless, an improved understanding of the growth kinetics of REN systems helps in possible tuning of material properties by controlling growth parameters effectively.

\subsubsection{Effect of growth temperature}

Generally, in heteroepitaxial systems, the growth temperature is chosen to best facilitate epitaxial growth of the material to be grown; pure metallic films can often grow epitaxially at even room temperature but often high temperatures up to several hundred degrees Celsius are required for the best quality epitaxial films, e.g. in the growth of III-nitrides (GaN, AlN). Elevated substrate temperatures allow adatoms that condense from the gaseous phase onto the substrate to diffuse and be incorporated into the growing film. It is also possible for adatoms to re-evaporate, which is especially significant for the growth of $\mathrm{SmN}$ as metallic Sm has the second highest volatility among the rare earths behind Eu. The effect of re-evaporation as a function of growth temperature is visible looking at the growth rate as measured from the thicknesses of the grown films (Fig. 5.13). The growth rate is noted to drop as the substrate temperature is increased above $500^{\circ} \mathrm{C}$, and actually tends to zero when using $\mathrm{N}_{2}$ as the nitrogen precursor. Growth with $\mathrm{NH}_{3}$ is 


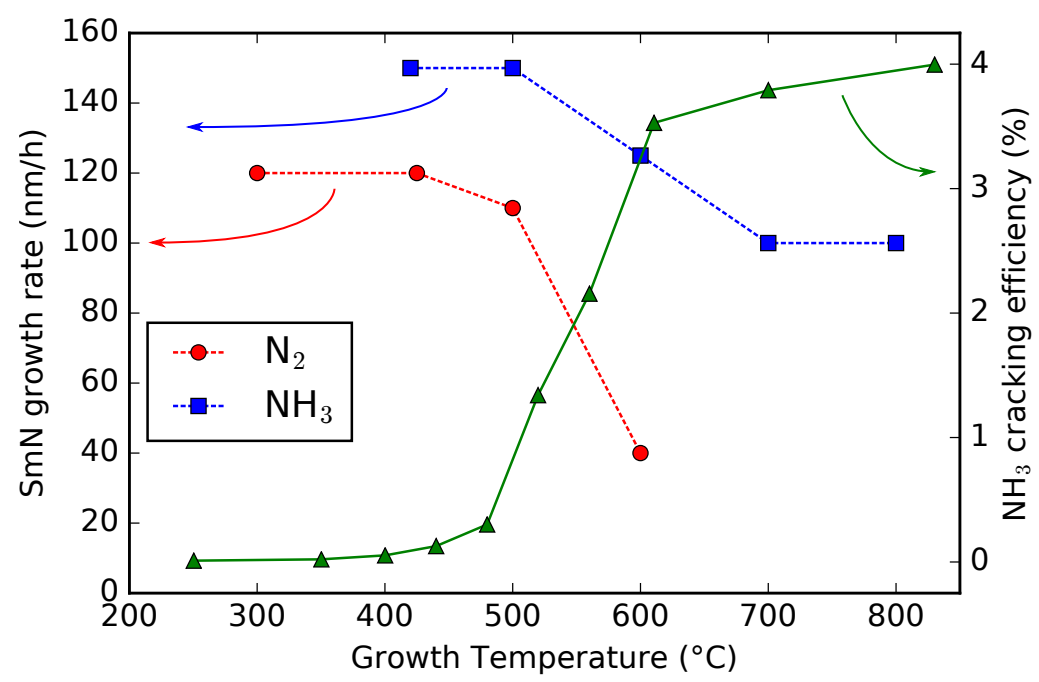

Figure 5.13: SmN growth rate as a function of growth temperature for films grown using the nitrogen precursors $\left(\mathrm{NH}_{3}\right.$, blue squares and $\mathrm{N}_{2}$, red dots). The thermal cracking efficiency of $\mathrm{NH}_{3}$ as measured in the case of MBE growth of GaN [75] is simultaneously plotted (green triangles). The SmN growth rate was determined from cross-section scanning electron microscopy thickness measurements. 
able to continue up to $800{ }^{\circ} \mathrm{C}$ where the growth rate has only dropped by $30 \%$ compared to the maximum growth rate at lower temperatures. Noting that

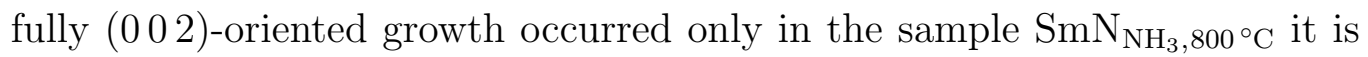
reasonable to think that the Sm re-evaporation (which is limited in the case of $\mathrm{NH}_{3}$ growth) is a contributing factor to the growth orientation transition. Further discussion of the differences in nitrogen precursor are shown below including the effect of thermal cracking of $\mathrm{NH}_{3}$.

\subsubsection{Energy considerations}

The competition between surface and interface energies is important to consider what epitaxial growth modes are favourable. With regards to the epilayer surface energy in the case of $\mathrm{SmN}$ and indeed other ionic rock salt structures, the (111) surface consisting entirely of either cations or anions is unstable due to a diverging surface energy [76]; the real surface must be stabilised in some way, e.g. by a surface reconstruction; in contrast, the neutral (001) is the lowest energy surface for ionic rock salt materials as it only has a single dangling bond.

Due to the large lattice mismatch between $\mathrm{SmN}$ and $\mathrm{AlN}$ in both (00 01 ) and (111) growth orientations, the initial film growth is not expected to be elastically strained to match the spacing in the substrate, i.e. the critical thickness below which dislocations are introduced to accommodate the strain is below one monolayer. One way this is possible is by domain matching epitaxy [77] where integer multiples of lattice planes are considered instead of just simply the atomic spacings used in the calculation of lattice mismatch.

Fig. 5.14 highlights possible extended domain matches for both epitaxial relationships shown in Eqs. 5.2 and 5.3. For (111)-oriented $\mathrm{SmN}$ growth on AlN (0001), a 7/8 domain matching has been identified along the AlN $\langle 11 \overline{2} 0\rangle$ direction, i.e.

$$
7 \times a_{\mathrm{SmN}(111)}=8 \times a_{\mathrm{AlN}}
$$

where $a_{\mathrm{SmN}(111)}=a_{\mathrm{SmN}} / \sqrt{2}$ and $a_{\mathrm{AlN}}$ are the in-plane lattice parameters of 

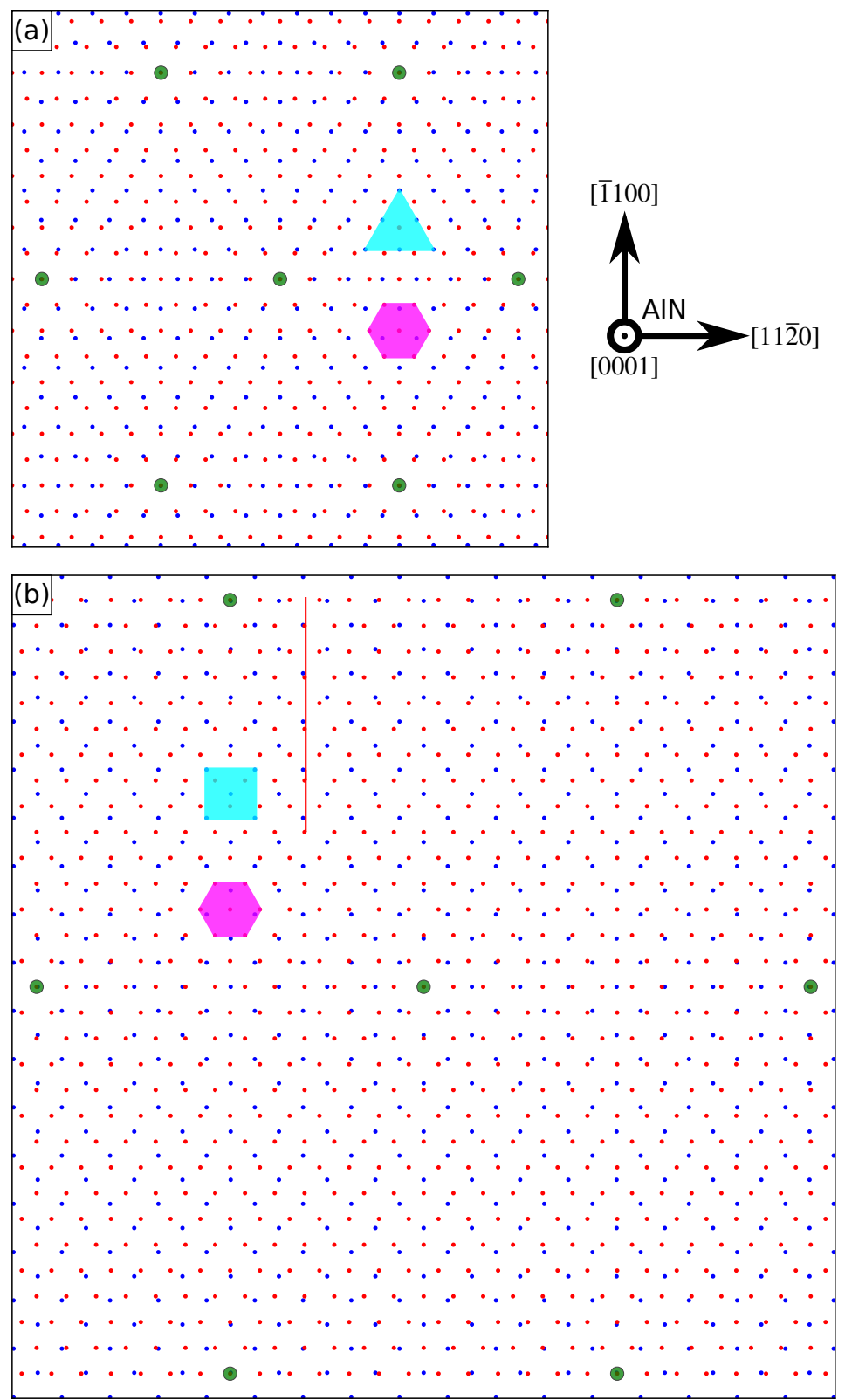

Figure 5.14: 2D schematic illustration highlighting the domain matching for the (a) (111) and (b) (001) growth orientations of SmN (blue dots representing Sm atoms) on the AlN (lo 0001 ) surface (red dots representing $\mathrm{Al}$ atoms). Only one rotational domain is shown for each growth orientation for clarity. The corresponding surface unit cells are shaded in cyan and magenta and coincidence sites highlighted in green. 
SmN (1 11 ) and AlN $\left\{\begin{array}{llll}0 & 0 & 0 & 1\end{array}\right\}$ respectively.

The case of (001)-oriented growth on $\mathrm{SmN}$ is interesting in that it involves the comparison of mismatched symmetries ("cube-on-hexagon") but it is still possible to identify two distinct domain matchings along AlN[11-20] and AlN[-1100] respectively, namely

$$
\begin{aligned}
8 \times a_{\mathrm{SmN}(001)} & =13 \times a_{\mathrm{AlN}} \\
16 \times a_{\mathrm{SmN}(001)} & =15 \sqrt{3} \times a_{\mathrm{AlN}}
\end{aligned}
$$

The existence of these domain matchings allows strain-free growth to occur if all misfit dislocations are concentrated at the interface which RHEED suggests to be the case. The fact that the identified domain matching for $\mathrm{SmN}(001)$-oriented growth is in some sense larger than the one for $\mathrm{SmN}$ (111) agrees with the observed growth orientation transition as the increased diffusion at higher growth temperatures can provide long range order needed for the larger domain matching.

\subsubsection{Nitrogen precursor dependence}

The ability to grow most of the RENs simply by exposing a substrate to a flux of RE metal atoms in the presence of molecular $\mathrm{N}_{2}$ is somewhat surprising given the strength of the $\mathrm{N}-\mathrm{N}$ triple bond which must be broken down catalytically during the growth in a process of dissociative chemisorption. It is similarly possible to catalytically break $\mathrm{NH}_{3}$ to allow its use as a nitrogen precursor in the growth of RENs; prior to this study it was thought that thermal cracking of $\mathrm{NH}_{3}$ was necessary for REN growth. The thermal cracking efficiency of $\mathrm{NH}_{3}$ has been measured for the case of GaN MBE (Fig. 5.13). The onset of the thermal cracking efficiency notably coincides with the decrease in SmN growth rate resulting from Sm re-evaporation and it is likely the increased access to atomic nitrogen provided by thermal cracking that limits the re-evaporation of $\mathrm{Sm}$ at the highest growth temperatures. In the case of $\mathrm{N}_{2}$ which has no thermal cracking component, the rate of Sm reevaporation is uninhibited as $\mathrm{N}_{2}$ cannot react quickly enough. We also note 
that for the case of $\mathrm{GaN} \mathrm{NH}_{3}-\mathrm{MBE}$, the thermal cracking efficiency is near zero below $500{ }^{\circ} \mathrm{C}$ but $\mathrm{SmN}$ growth was still able to progress at temperatures as low as the $425^{\circ} \mathrm{C}$ tested, evidencing the catalytic breaking of $\mathrm{NH}_{3}$ as well. This catalytic activity of Sm should result in an overall shift of the green curve in Fig. 5.13 towards lower temperatures.

With regards to the observed growth orientation transition, we look at the actual atomic structure of the $\{111\}$ and $\left\{\begin{array}{lll}0 & 0 & 1\end{array}\right\}$ planes of the rock salt structure of SmN. The $\{111\}$ family of planes consists of close-packed layers that alternate between fully consisting of Sm and N. For growth with $\mathrm{N}_{2}$ (i.e. by catalytic dissociative chemisorption) in particular, one might reasonably expect that $\langle 111\rangle$ is thus heavily favoured as a growth direction as it provides the largest surfaces of $\mathrm{Sm}$ for $\mathrm{N}_{2}$ to adsorb and catalytically dissociate with. Additionally, initial nucleation of (111)-oriented crystallites on the AlN substrate would be favoured to initiate $\mathrm{N}_{2}$ adsorption and incorporation.

In contrast, the $\left\{\begin{array}{lll}0 & 0 & 1\end{array}\right\}$ plane of $\mathrm{SmN}$ is a mix of $\mathrm{Sm}$ and $\mathrm{N}$ atoms arranged in a square lattice. The planar density of a single species of atom compared to the close-packed $\{111\}$ planes is only $61 \%$. It is possible that the subsaturation caused by re-evaporation of Sm at high temperatures combined with the thermal cracking of $\mathrm{NH}_{3}$ allowing access to atomic nitrogen accommodates initial nucleation of ( 001$)$-oriented crystallites in combination with the energy considerations above.

\subsubsection{Other members of the REN series}

Evidence for a similar growth orientation transition for any other members of the REN series has yet to be obtained, however, as Eu which is even more volatile than $\mathrm{Sm}, \mathrm{EuN}$ is the most likely candidate to exhibit similar behaviour in the same growth temperature ranges. Epitaxial EuN has been grown at high temperatures in an adsorption-controlled growth regime to evaporate excess Eu on (0001) AlN and GdN [25], but this was performed using activated $\mathrm{N}_{2}$ with a Kaufman ion source rather than $\mathrm{NH}_{3}$ as was used for the growth of SmN here, and no evidence of multiple epitaxial growth 
orientations was reported. For other members of the REN series, much higher growth temperatures than can be reasonably achieved would be required to result in similar reevaporation of $\mathrm{RE}$ adatoms.

\subsection{Conclusion}

The effect of the growth temperature and choice of nitrogen precursor has been studied for the epitaxial integration of SmN on AlN (0001) surfaces. A transition in the growth orientation from the normally observed (111)oriented $\mathrm{SmN}$ to (0 01 )-oriented), controllable by growth temperature is observed through diffraction techniques, RHEED and XRD. Such a growth orientation transition is especially unusual due to the tendency of rock salt materials to match the close-packed (111) plane with the (0001) surface of the AlN substrate. The surface of epitaxially grown $\mathrm{SmN}$ has been directly imaged for the first time using STM to probe the surface morphology with even atomic steps being resolved. The nitrogen precursor dependence of this growth orientation transition as well as other measurements of the crystalline quality and surface morphology are used to try to understand the fundamental driving factors in the growth process that are able to lead to this unusual system. The thermal cracking process that can provide enhanced access to atomic nitrogen during growth for growth by $\mathrm{NH}_{3}$ is thought to be a key factor in allowing fully (0 01 )-oriented growth, combined with the reevaporation of adsorbed Sm and the viability of the $\left\{\begin{array}{lll}0 & 0 & 1\end{array}\right\}$ orientation under energetic considerations despite the mismatch in symmetry. 


\section{Chapter 6}

\section{Removable Samarium capping layer}

\subsection{Introduction}

A major challenge to overcome when studying the REN series is their propensity to decompose and oxidise in air. Films are generally capped with a passivating layer to prevent reaction with the ambient atmosphere. Commonly used passivating layers include amorphous or polycrystalline AlN and GaN as they have good chemical stability and their transparency allows optical measurements. Other materials have also been successfully tried in the past [7], including metallic layers such as $\mathrm{W}, \mathrm{Cr}, \mathrm{Cu}$, TaN, NbN and insulating layers such as YSZ and MgF.

These capping layers have been effective in allowing characterisation of REN thin films through techniques such as XRD and SQUID magnetometry to get information about the bulk magnetic, electronic, optical and structural properties of deposited films [7].

Such capping layers, however, do not allow for various fundamental characterisation techniques that require direct access to the REN film, such as ARPES or XPS which could give even further information about the electronic structure or elemental composition of the surface, or other surface 
science tools like STM for morphology characterisation. For the study of air-sensitive thin films, specialised equipment is required where a growth chamber is coupled under vacuum to the desired experimental apparatus; this becomes increasingly prohibitive as more experimental techniques are needed.

One way to get around this requirement of specialised systems is a temporary capping layer, allowing a sample to be grown separately in one vacuum system and transferred to the second vacuum system with the experimental apparatus in which the capping layer would be removed under vacuum exposing the REN surface for characterisation.

An example of this process being implemented is arsenic passivation of epitaxially grown GaAs $[78,79]$; the GaAs surface can be restored by heating the sample to $\sim 350^{\circ} \mathrm{C}$ in UHV.

Regarding the study of the RENs, we thus need to find a similarly suitable material to act as the temporary capping layer and develop an in situ decapping process that does not significantly modify the properties of the underlying REN layer allowing probing of the surface. For a capping layer to be removed thermally, the material would ideally be volatile such that it easily sublimes under moderate applied heat in vacuum. It would also have to avoid reacting with the REN layer and avoid contaminating the growth system. For example, while Mg fulfils the first property, the well-known "memory effect" from Mg coating the growth chamber walls contaminates later growths resulting in highly resistive films from unintentional doping $[26$, 61].

We investigate the possibility of using metallic Sm layers as removable capping layers for REN samples. Sm has the second highest volatility in the rare earths behind $\mathrm{Eu}$, giving rise to the possibility of thermally desorbing a Sm layer at moderate temperatures. Additionally, Sm sources are already present or otherwise easily accommodatable in systems used to grow REN samples. We investigate the properties of Sm layers grown on top of epitaxial GdN layers. The process for thermal removal is also developed, and the 
quality of the remaining layer and affect on properties of the underlying GdN layer are studied.

A patent has been filed before the European Patent Office concerning the work in this chapter:

[14]F. Natali, B. J. Ruck, J. H. Trodahl, and J. R. Chan. "Rare Earth

Nitride Structures and Devices and Method for Removing a Passivating

Capping". European Patent 16202663.7 (Filed 07 December 2016)

\subsection{Properties of elemental Sm}

$\mathrm{Sm}$ is the 6th member of the lanthanide series with atomic number 62. It is a silvery metal that is paramagnetic at room temperature and undergoes antiferromagnetic transitions at $106 \mathrm{~K}$ and $15 \mathrm{~K}$ and it has a number of industrial applications in bulk form, one being as a component in high-temperature permanent magnets used for example in power generation [80].

\subsubsection{Structure}

At ambient conditions, bulk Sm crystallises in a hexagonal close-packed structure with a complicated 9-layer "Sm-structure" stacking sequence (Fig. 6.1 $)$, but can undergo phase transitions under pressure to hcp, dhcp (shown in Fig. 6.1 b), fcc and bcc structures under elevated temperatures and pressures [81]. Growth of thin films of Sm by MBE under the right conditions can also lead to almost pure dhcp single crystalline films [82].

\subsubsection{Reactivity}

Thin films of Sm metal have been measured to oxidise following a parabolic rate law 83$]$ :

$$
T=2.24 t^{1 / 2}+1.3
$$




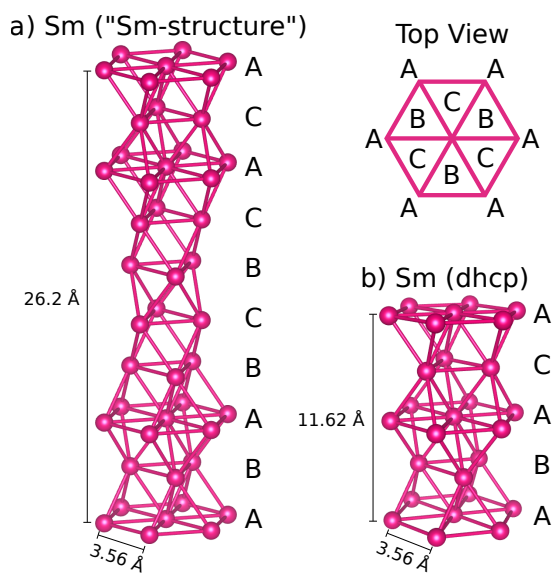

Figure 6.1: The two observed hexagonal close-packed structures of elemental Sm. (a) The eponymous 9-layer "Sm-structure" stacking sequence (ABABCBCAC $\mid \mathrm{AB} \ldots$ ) is compared to (b) the four layer dhcp structure (ABAC $\mid$ AB...). The relative positions of the different layers is shown in the top right.

where $T$ is the oxide thickness in $\mathrm{nm}$ and $t$ the time in days; a $100 \mathrm{~nm}$ thin film was found to form an oxide layer of $17 \mathrm{~nm}$ after 2 months in air at room temperature and reaching $35 \mathrm{~nm}$ in 6.5 months. The effectiveness of $\mathrm{Sm}$ as a passivating layer for RENs is demonstrated below when combined with standard REN sample storage under vacuum limiting the total exposure to minutes or hours; we also investigate the effect of any oxidation on the ability to thermally remove the capping layer.

\subsubsection{Sublimation of bulk Sm}

A first estimation of the sublimation rate of a material in a perfect vacuum (i.e. zero external pressure) heated to a temperature $T$ is obtained using the Hertz-Knudsen equation [84]:

$$
\frac{1}{A} \frac{d N_{e}}{d t}=\left(2 \pi m k_{B} T\right)^{\frac{1}{2}} P
$$




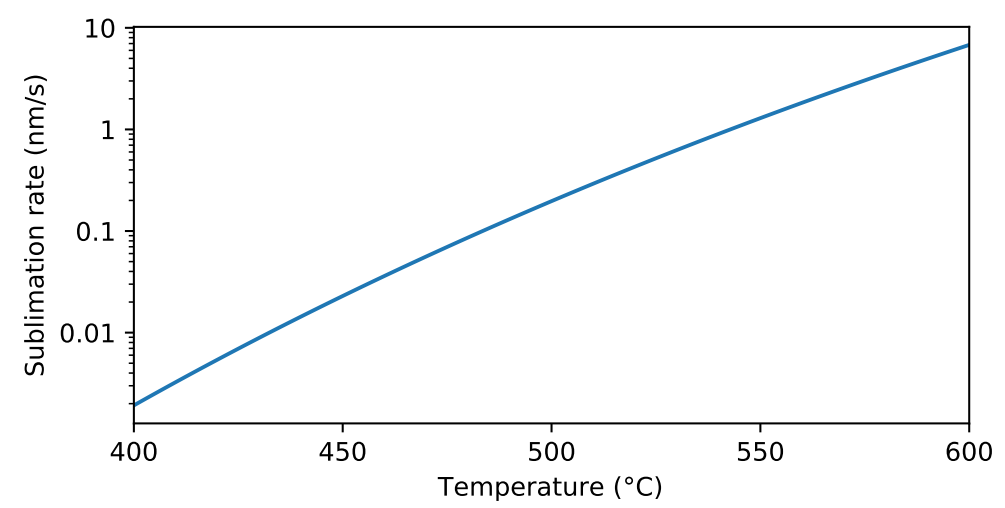

Figure 6.2: The sublimation rate of bulk $\mathrm{Sm}$ as a function of temperature, calculated using Eqs. 6.2 and 6.3. A temperature of $550{ }^{\circ} \mathrm{C}$ gives an order of magnitude for the sublimation rate of $1 \mathrm{~nm} \mathrm{~s}^{-1}$ from the surface.

where $d N_{e} / d t$ is number of sublimed atoms or molecules per unit time, $A$ the surface area, $m$ mass of atom/molecule, $k_{B}$ the Boltzmann constant and $P$ the equilibrium vapour pressure of the material at temperature $T$.

Using experimental data, the vapour pressure of Sm as a function of temperature can be calculated empirically using an extended Antoine equation with the following parameters 85 ,

$$
\log (P / \mathrm{Pa})=14.994-11034 /(T / \mathrm{K})-1.3287 \log (T / \mathrm{K})
$$

and using the atomic number density of Sm, $N_{\mathrm{Sm}}=30$ atoms $/ \mathrm{nm}^{3}$, the sublimation rate in $\mathrm{nm} \mathrm{s}^{-1}$ calculated for a temperature range of $400{ }^{\circ} \mathrm{C}$ to $600^{\circ} \mathrm{C}$ is shown in Fig. 6.2 in this range, the sublimation rate increases by an order of magnitude roughly every $60^{\circ} \mathrm{C}$, and the estimation suggests that $50 \mathrm{~nm}$ of Sm could be evaporated in about 40 minutes using a temperature of $450^{\circ} \mathrm{C}$; although the evaporation of a thin film is almost certainly going to deviate from this bulk estimation these values can act as a starting point for attempting thermal desorption. 


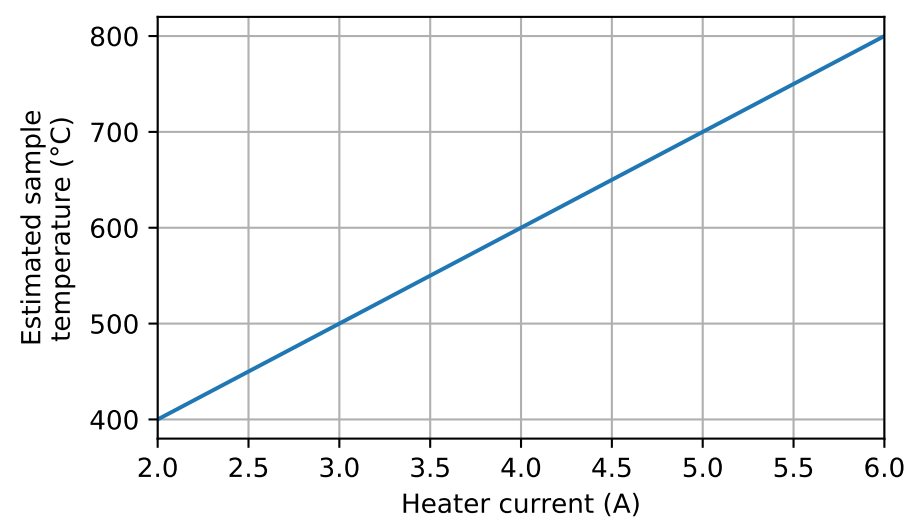

Figure 6.3: The estimated temperature of a sample inside the Thermionics UHV chamber as a function of current through the heater filament.

\subsection{Sample growth and measurement}

The samples in this study were grown at VUW using the UHV MBE system. All epitaxial GdN samples were grown on Si/AlN (0001) templates with a typical GdN growth rate of $60 \mathrm{~nm} \mathrm{~h}^{-1}$ using molecular $\mathrm{N}_{2}$ with a growth pressure of $1.0 \times 10^{-4}$ mbar. GdN layer thicknesses were between $20 \mathrm{~nm}$ to $100 \mathrm{~nm}$. The deposition rate of Sm metal was about $150 \mathrm{~nm} \mathrm{~h}^{-1}$ with Sm layer thicknesses between $50 \mathrm{~nm}$ to $80 \mathrm{~nm}$. RHEED was used during the growths to confirm the epitaxial nature of the growth and the thermal desorption processes tested.

For epitaxial growth and the annealing process, a heater filament inside the UHV chamber is used to raise the temperature of the sample. The temperature of the sample inside the chamber cannot be measured directly; instead, henceforth we specify the current through the filament. The temperature is known to approximately vary linearly with current above $2 \mathrm{~A}$. An estimate of the sample temperature as a function of applied current was obtained using an optical pyrometer based on the Si (111) $7 \times 7$ surface reconstruction (Fig. 6.3). 


\subsection{Stability of GdN}

Initial concerns about the stability of GdN layers at high temperatures above $500{ }^{\circ} \mathrm{C}$ under vacuum had to be addressed to make sure the thermal decapping process wouldn't strongly affect the quality of the remaining GdN layer. It would not be entirely unexpected for a high enough annealing temperature to negatively affect the surface morphology of the GdN film by recrystallisation or nitrogen loss; such processes are known to occur in other nitride semiconductor compounds such as GaN [86, 87]. To that end, the RHEED pattern of as-grown GdN was measured while heating the sample up under UHV with $\mathrm{N}_{2}$ off to try to identify any noticeable degradation of the surface.

An epitaxial GdN layer of thickness about $100 \mathrm{~nm}$ was grown on an $\mathrm{Si} / \mathrm{AlN}$ template and the chamber allowed to pump down to base pressure $\left(\sim 2.0 \times 10^{-8}\right.$ mbar $)$ overnight without removing the sample. Before beginning the annealing process, some Gd was evaporated into the chamber while the sample was protected with a shutter to prevent depositing any on the sample surface. Evaporating Gd in this way acts as a sublimation pump by reacting with components of the residual gas inside the chamber to further reduce the pressure to $6.0 \times 10^{-9}$ mbar. A quadrupole residual gas analyser was used to monitor the gas composition during the annealing process. The sample was annealed using a heater current of $4.5 \mathrm{~A}\left(\sim 650^{\circ} \mathrm{C}\right)$ for 1 hour, the highest temperature that was to be tested during the thermal desorption.

Fig. 6.4 shows RHEED images taken before and after the annealing process along the $\operatorname{GdN}\langle 1 \overline{1} 0\rangle$ azimuth. No obvious change in the surface morphology is visible in the RHEED pattern; the diffraction spots remain the same after the annealing process showing no change in the surface roughness or in-plane lattice parameter of GdN.

The gas composition during the annealing process is shown in Fig. 6.5 with the partial pressures from $\mathrm{N}_{2}(\mathrm{CO})$ and $\mathrm{H}_{2}$ displayed. During the annealing process (while the sample heater is on), the $\mathrm{H}_{2}$ partial pressure increases from $10^{-10}$ mbar to $10^{-8}$ mbar, representing the largest change in gas composition. The $\mathrm{N}_{2}$ partial pressure similarly increases while the heater is 


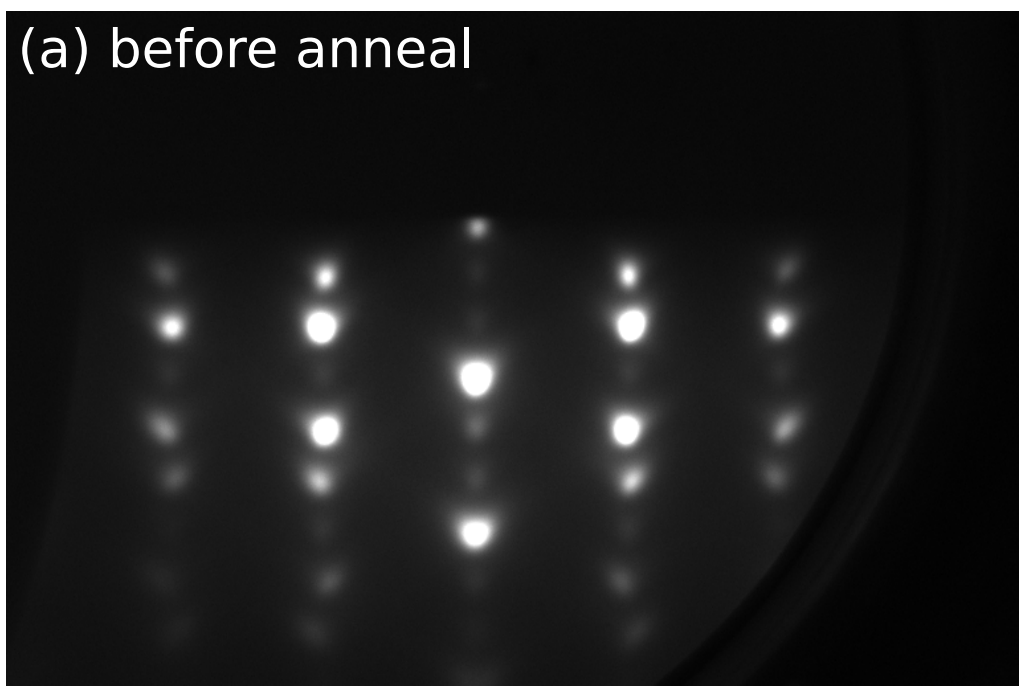

\section{(b) after anneal}

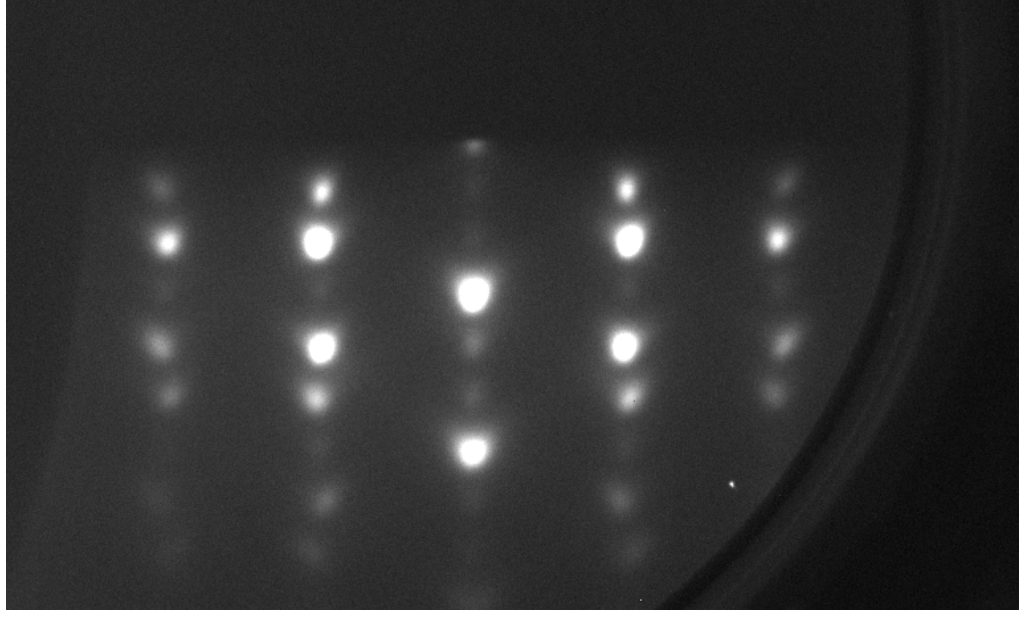

Figure 6.4: RHEED images taken of as-grown epitaxial GdN along the $\langle 1 \overline{1} 0\rangle$ azimuth before (top) and after 50 minutes of annealing at $4.5 \mathrm{~A}\left(\sim 650^{\circ} \mathrm{C}\right)$ in a chamber pressure of $6.0 \times 10^{-9}$ mbar. No obvious degradation of the sample (e.g. formation of polycrystalline rings) is observed as a consequence of the annealing process. 


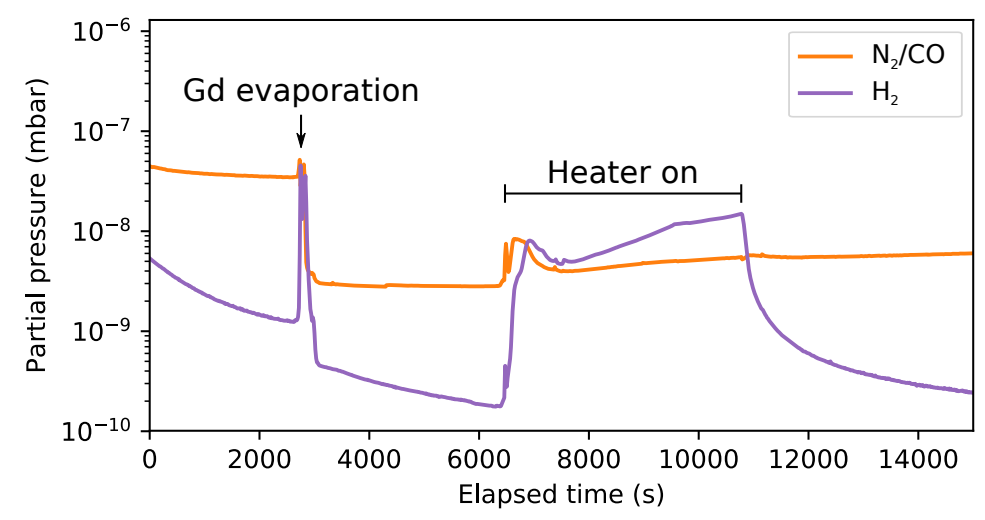

Figure 6.5: Residual gas partial pressures inside UHV chamber during annealing of as-grown GdN.

on, although the increase is not as dramatic.

\subsection{Epitaxial Sm growth on GdN}

\subsubsection{Structural properties}

As noted in Section 6.2, Sm metal is seen to crystallise mainly in hexagonal close packing lattices. The $a_{\text {Sm }}$ lattice constant with a value of about $3.56 \AA$ has a small lattice mismatch with the GdN (111) surface of only $1.1 \%\left(a_{\mathrm{GdN}(111)}=3.52 \AA\right)$. RHEED images of Sm deposited on the as-grown GdN (111) at room temperature (Fig. 6.6) show that the Sm layer growth is epitaxial and notably the resulting surface roughness is much lower than the initial GdN roughness as indicated by the streakier RHEED pattern.

In most samples, satellite streaks corresponding to a $(5 \times 5)$ surface reconstruction are observed (Fig. 6.6). This has been observed in other epitaxial growths of Sm and is related to the divalence of surface Sm [82, 88]. The conditions for the appearance of this surface reconstruction are unclear in the literature but likely related to the crystalline quality of the deposited film.

XRD was used to study the structural properties of the Sm layers. Sym- 


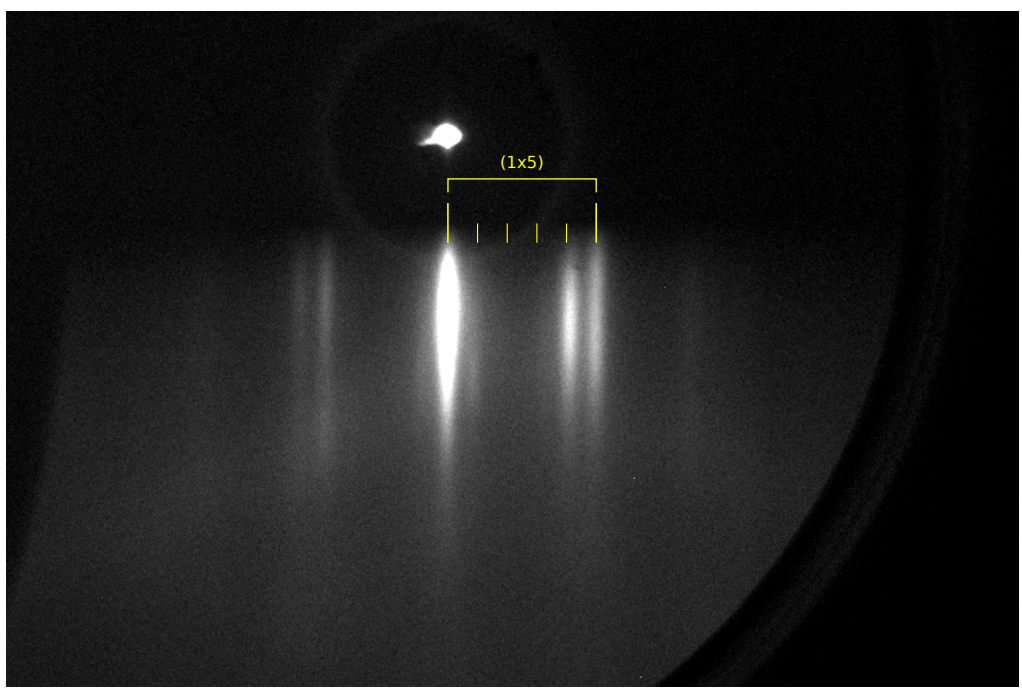

Figure 6.6: RHEED pattern of a Sm capping layer deposited on epitaxial GdN along the $\langle 11 \overline{2} 0\rangle$ azimuth. The diffraction pattern is streaky compared to the typical rough GdN surface observed with RHEED (Fig. 6.4), indicating that the resulting Sm surface is smooth despite the initial roughness. The $(1 \times 5)$ periodicity satellite streaks from the surface reconstruction are indicated in yellow.

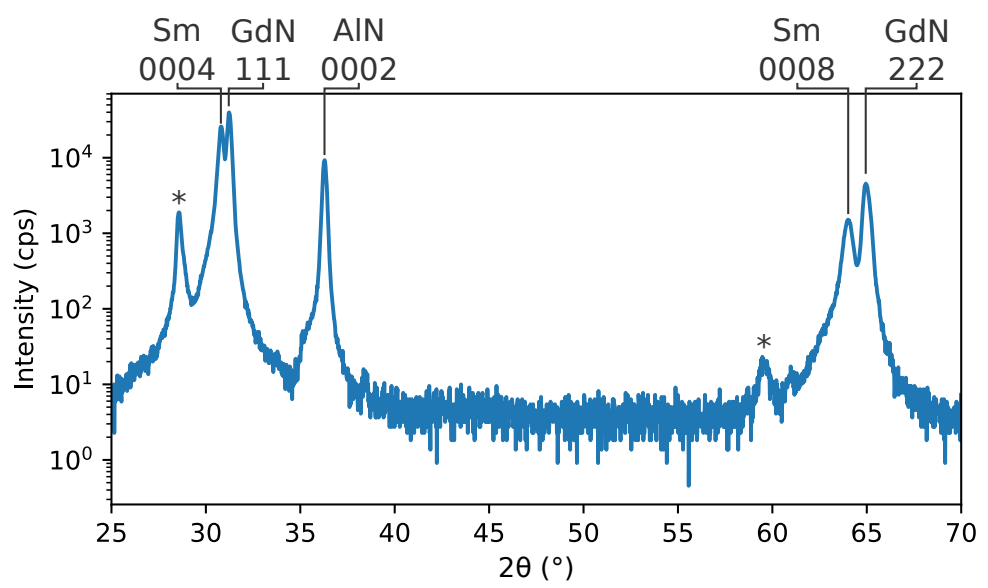

Figure 6.7: $2 \theta-\omega$ XRD scan of a Sm-capped epitaxial GdN sample grown on AlN. Si substrate peaks are marked with asterisks. The sample consists of fully (111)-oriented GdN and (0 0001$)$-oriented Sm. 


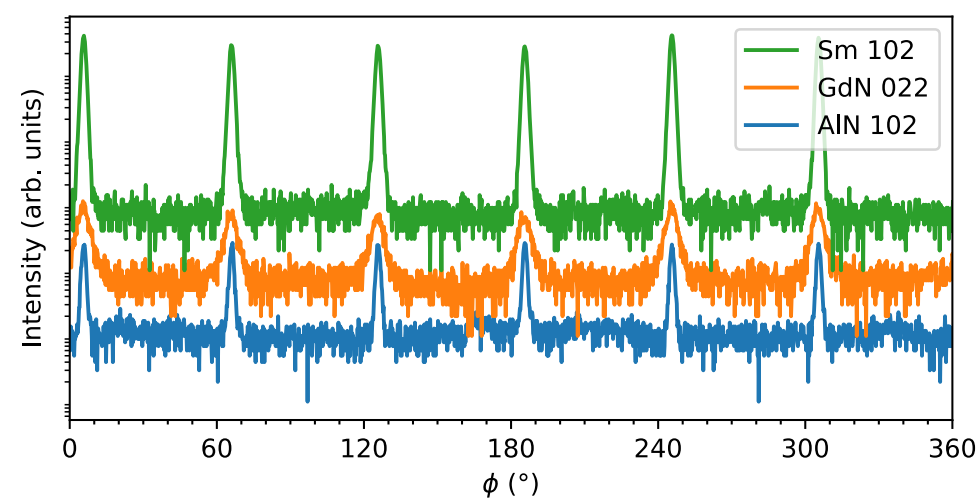

Figure 6.8: XRD $\phi$ scans of a Sm-capped epitaxial GdN sample grown on AlN. All three reflections from the respective layers exhibit 6-fold symmetry.

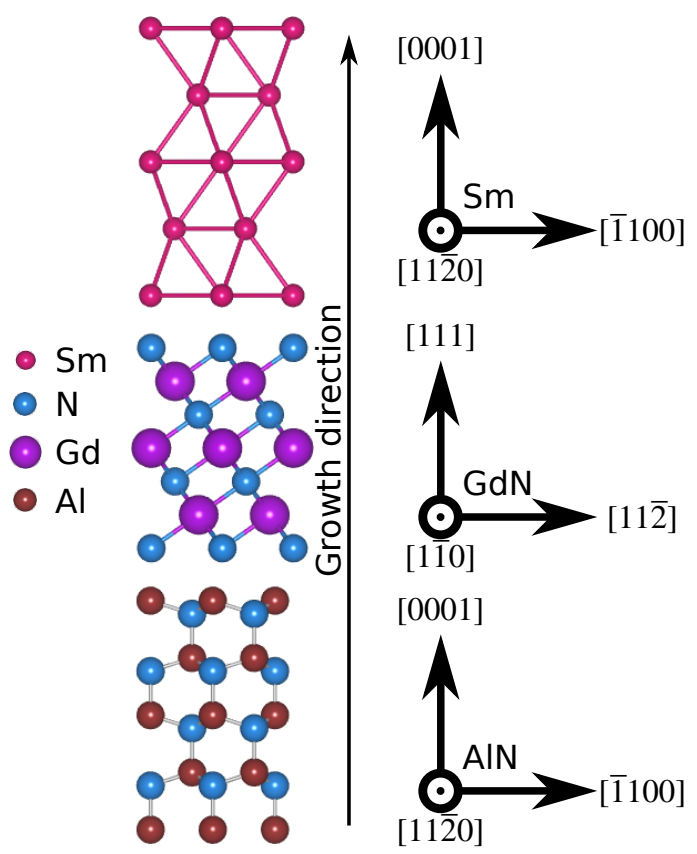

Figure 6.9: Atomic representation showing a cross-section of the observed epitaxial relationship between AlN, GdN and Sm 6.4 as inferred from XRD $\phi$ scans (Fig. 6.8). 


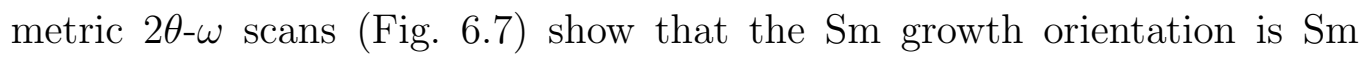
(0001) parallel to the GdN (111) layer beneath, although this does not provide enough information to distinguish between stacking sequences, i.e. whether the bulk film is dhcp or "Sm-structure" or some mix thereof. Asymmetric $\phi$ scans of Sm $10 \overline{1} x$ compared to the GdN 022 reflection reveal that the full epitaxial relationship between the AlN, GdN and Sm layers is

$$
\begin{aligned}
& \operatorname{Sm}\left(\begin{array}{lllll}
0 & 0 & 0 & 1)
\end{array}\right) \operatorname{GdN}\left(\begin{array}{llll}
1 & 1 & 1
\end{array}\right) \| \operatorname{AlN}\left(\begin{array}{llll}
0 & 0 & 0 & 1
\end{array}\right) \\
& \operatorname{Sm}(0001)\langle 11 \overline{2} 0\rangle\left\|\operatorname{GdN}\left(\begin{array}{lll}
1 & 11 & 0
\end{array}\right)\langle 1 \overline{1} 0\rangle\right\| \operatorname{AlN}\left(\begin{array}{llll}
0 & 0 & 0 & 1
\end{array}\right)\langle 11 \overline{2} 0\rangle
\end{aligned}
$$

An atomic schematic of this relationship is shown in Fig. 6.9 showing a cross section of the layer structure of the films.

The precise location of the Sm $10 \overline{1} x$ peak measured in the $\phi$ scan allows distinguishing of the two crystal structures of Sm. The Sm peak was observed at a sample tilt of $62.1^{\circ}$ at a d-spacing of $2.764 \AA$; this peak is only present in the dhcp structure of $\mathrm{Sm}$ and corresponds to the $10 \overline{1} 2$ reflection. The measured lattice constants are $a=3.56 \AA$ and $c=11.62 \AA$. This compares well with measurements of the lateral lattice spacing from RHEED; the streak spacing for the Sm capping layer shows an expansion of about $1 \%$ compared to the GdN lateral lattice spacing of $3.52 \AA$.

\subsubsection{Electric and magnetic properties}

The temperature dependence of the resistivity of a typical Sm-capped GdN sample measured ex situ is shown in Fig. 6.10. It resembles closely that reported for pure films of polycrystalline Sm metal, suggesting little contribution from the GdN layer below. Anomalous behaviour in the temperature dependence is attributed to antiferromagnetic transitions in Sm around $100 \mathrm{~K}$ and $10 \mathrm{~K} 89$. .

Magnetic characterisation of a Sm-capped epitaxial GdN thin film is shown in Fig. 6.11. The film thicknesses for this sample were $70 \mathrm{~nm}$ and $50 \mathrm{~nm}$ for GdN and Sm respectively. A field cooled curve is shown in Fig. 6.11. The sample is cooled from above the magnetic transition temperature to $4 \mathrm{~K}$ in 


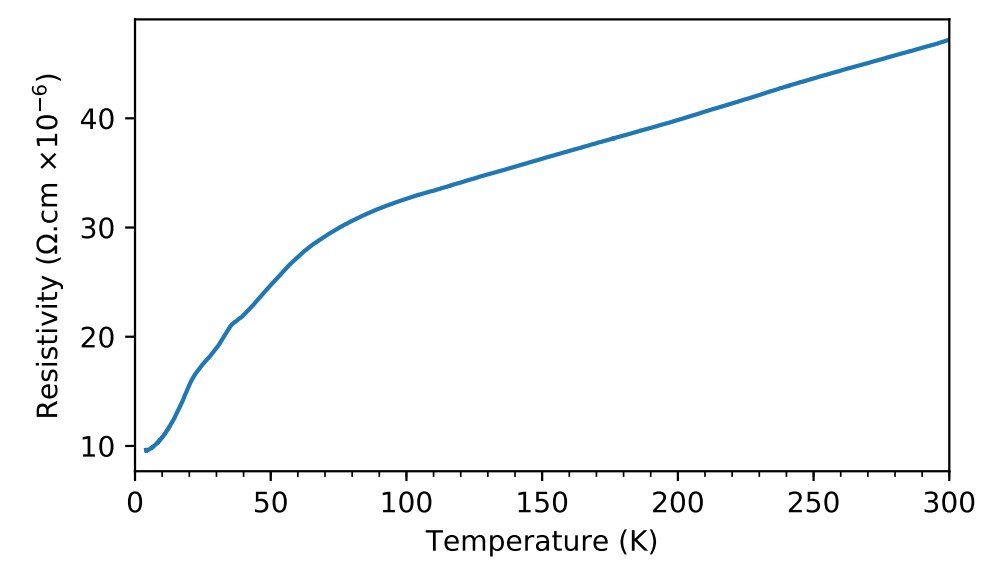

Figure 6.10: Temperature dependent resistivity of an epitaxial GdN sample grown on AlN and capped with Sm metal. The resistance decreases in a linear fashion from $300 \mathrm{~K}$ down to about $100 \mathrm{~K}$. The change in behaviour below $90 \mathrm{~K}$ is due to an antiferromagnetic transition of $\mathrm{Sm}$.

an applied field of 25 Oe while recording the magnetisation of the thin film sample. The inverse of the susceptibility (Fig. 6.11) shows that the ferromagnetic transition temperature $T_{C}$ is approximately $67 \mathrm{~K}$. Field dependent measurements (Fig. 6.11 c and d) scan from positive to negative applied fields while the sample is at a fixed temperature and show that the coercive field is about 100 Oe at the lowest temperature of $5 \mathrm{~K}$. These values are similar to those observed in previously grown epitaxial GdN thin films 35, 36], suggesting no significant change in the magnetic properties due to the Sm capping layer.

\subsection{Thermal desorption of Sm}

The study of the thermal desorption of the Sm capping layer has been divided into two cases: annealing of deposited Sm capping layers without removing the sample from the growth chamber after deposition; i.e. without exposing the Sm to air, and annealing of Sm-capped samples that have been exposed 

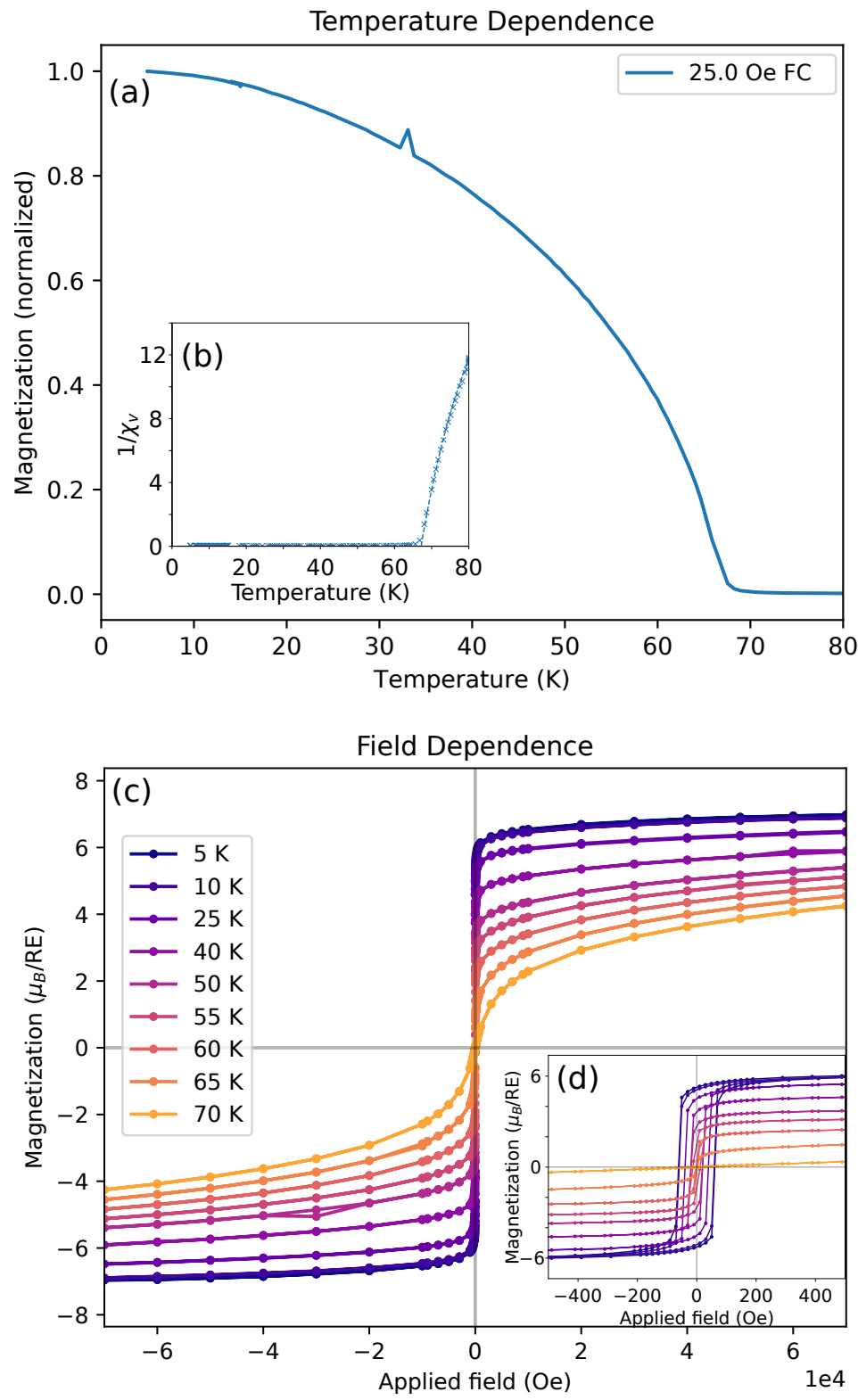

Figure 6.11: Temperature and field dependent magnetic measurements of an epitaxial GdN sample capped with Sm. (a) Field cooled curve using an applied field of 25 Oe and (b) corresponding inverse susceptibility showing a ferromagnetic transition with $T_{C}=67 \mathrm{~K}$. (c) Field dependence at different temperatures showing magnetic hysteresis and magnetic saturation of $\sim 7 \mu_{\mathrm{B}} / \mathrm{Gd}^{3+}$. (d) Zoomed inset of hysteresis close to zero field showing low coercivity of 60 Oe at $5 \mathrm{~K}$. 
to atmosphere. The latter case is ultimately the goal of a removable capping layer than can be transferred between UHV systems, although exposure to air may introduce issues to the desorption process; this will be discussed below.

\subsubsection{Annealing without exposure to atmosphere}

We first investigate the thermal desorption of as-deposited Sm capping layers that have not been exposed to ambient atmosphere to avoid the formation of a native oxide. During this process, the primary characterisation tool is in-situ RHEED.

An epitaxial GdN sample of thickness $60 \mathrm{~nm}$ was capped with approximately $100 \mathrm{~nm}$ of $\mathrm{Sm}$. Without being removed from the chamber, the Sm-capped sample was annealed using a heater current of $4.5 \mathrm{~A}\left(\sim 650^{\circ} \mathrm{C}\right)$. RHEED images taken at different times during the annealing process along the $\mathrm{Sm}\langle 11 \overline{2} 0\rangle$ azimuth are shown in Fig. 6.12. The relative lattice spacings as determined from the inverse of the primary streak spacing are plotted as a function of time since the start of annealing in Fig. 6.13. We observe an evolution of the RHEED pattern corresponding to the progress of the thermal desorption of the Sm capping layer. Remarkably, before the expected spacing of the GdN layer is recovered, an intermediate phase is observed in the RHEED pattern corresponding to a roughly $22 \%$ increase in lattice spacing. This increase in lattice spacing has been attributed to a surface layer of divalent Sm with a $22 \%$ larger atomic radius on top of the bulk trivalent $\mathrm{Sm}$ 90, 91, although this has not been observed at elevated temperatures before. The final RHEED pattern obtained after the annealing process has a slightly smaller streak spacing by roughly $2 \%$ than that seen at the start, close to the theoretical lattice mismatch of $1.1 \%$ between Sm and GdN, suggesting most of the Sm has been evaporated off the sample.

The above sample was capped with a layer of AlN for further ex-situ measurements. No traces of Sm metal were visible in a $2 \theta-\omega$ XRD scan (Fig. 6.14). The temperature dependence of the resistivity of the sample 

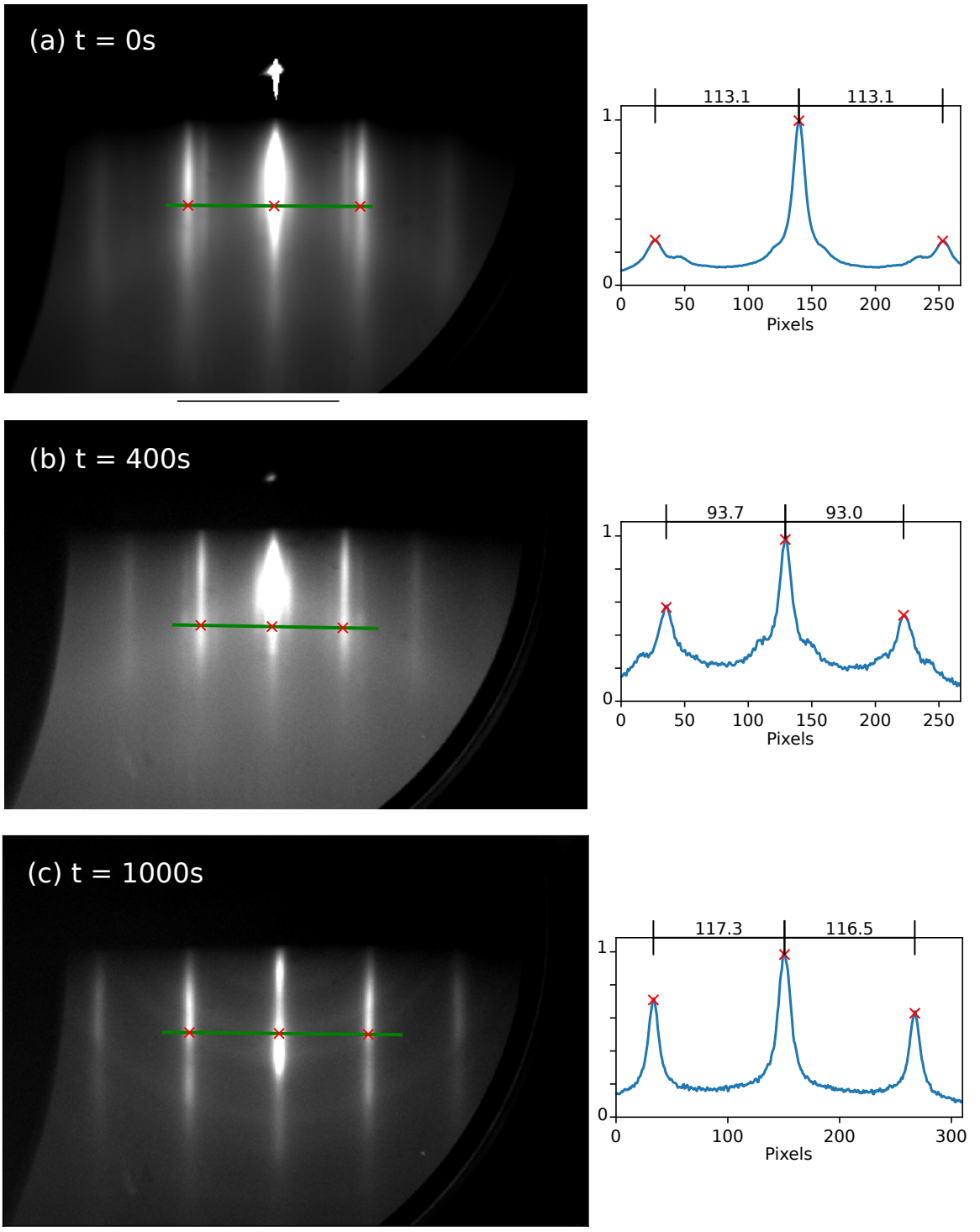

Figure 6.12: RHEED images taken during the annealing at $4.5 \mathrm{~A}$ $\left(\sim 650^{\circ} \mathrm{C}\right)$ of a Sm-capped GdN sample unexposed to atmosphere along the $\mathrm{Sm}\langle 11 \overline{2} 0\rangle$ azimuth. Line profiles are displayed to the right to show the peak spacings in pixels. At the start of the anneal (a), the RHEED image shows the Sm pattern with the $5 \times 5$ surface reconstruction visible. (b) About 6 minutes into the anneal, the intensity of the inner satellite streak strengthens, corresponding to a roughly $22 \%$ lattice expansion. (c) After 15 minutes, the streak spacing increases, thought to correspond mainly to GdN. 


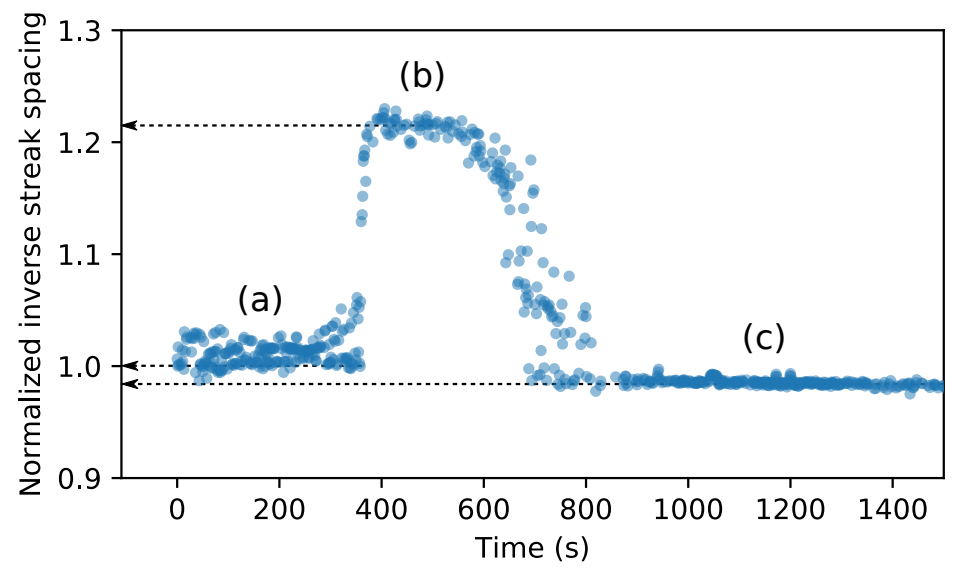

Figure 6.13: Variation of lattice spacing as measured by RHEED during the annealing of the Sm capping layer (Fig. 6.12) as a function of time. Three stages with distinct streak spacings are identified corresponding to relative lattice spacings of (a) 1, (b), 1.22 and (c) 0.98 .

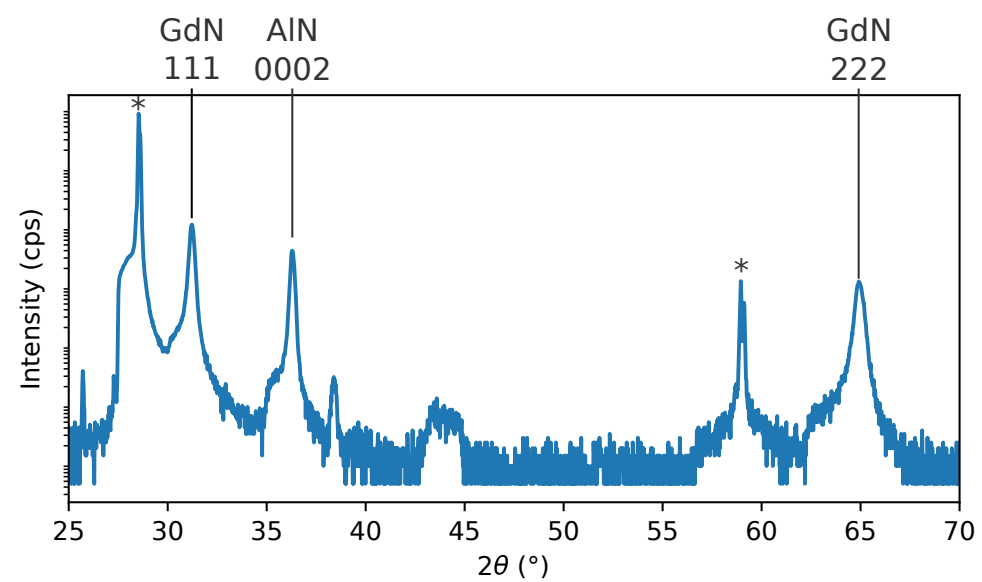

Figure 6.14: $2 \theta-\omega$ scan of an epitaxial GdN sample after being capped with Sm and undergoing an annealing process to thermally desorb the Sm. Only the GdN peaks remain visible (compare with Fig. 6.7) 


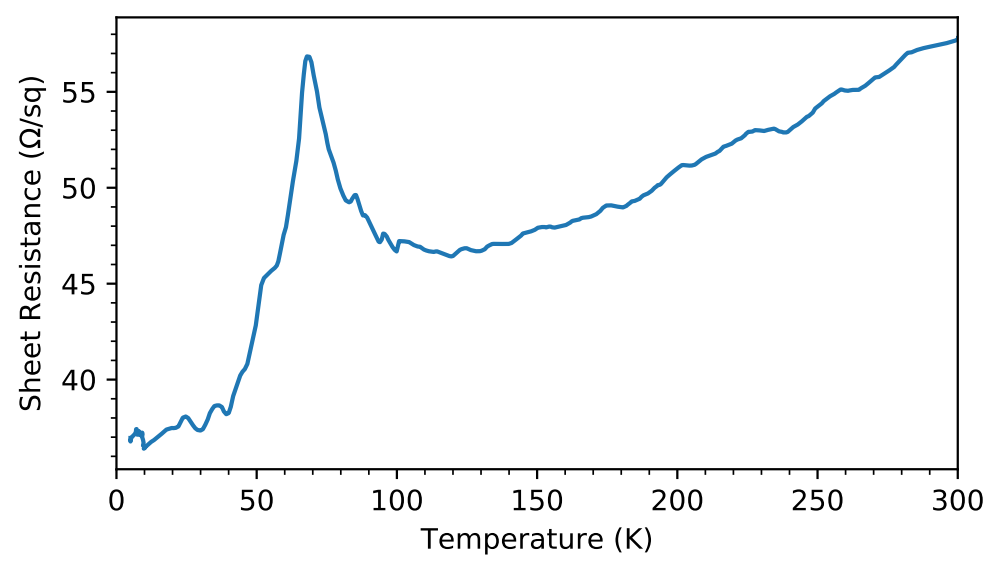

Figure 6.15: Temperature dependent sheet resistance of an epitaxial GdN sample after being capped with Sm and undergoing an annealing process to thermally desorb the Sm. The metallic behaviour characteristic of Sm thin films (Fig. 6.10) is not seen; the peak in resistivity corresponds to the ferromagnetic transition of GdN.

(Fig. 6.15) was consistent with the behaviour expected for a highly-doped epitaxial GdN thin film [35] instead of the metallic behaviour of a Sm thin film (Fig. 6.10). These results are consistent with successfully removing the bulk of the Sm capping layer.

Magnetic measurements of the same sample are shown in Fig. 6.16. The field cooled curve shows a measured Curie temperature of about $T_{C}=67 \mathrm{~K}$. Low temperature hysteresis measurements show that the saturation magnetisation of this sample is above $6 \mu_{\mathrm{B}} / \mathrm{Gd}^{3+}$ at $5 \mathrm{~K}$ with a coercive field of about 130 Oe. These magnetic measurements are comparable those shown in Fig. 6.11 and to previous magnetic measurements of epitaxial GdN [35], suggesting the annealing process has not significantly changed the magnetic properties of the GdN layer. 

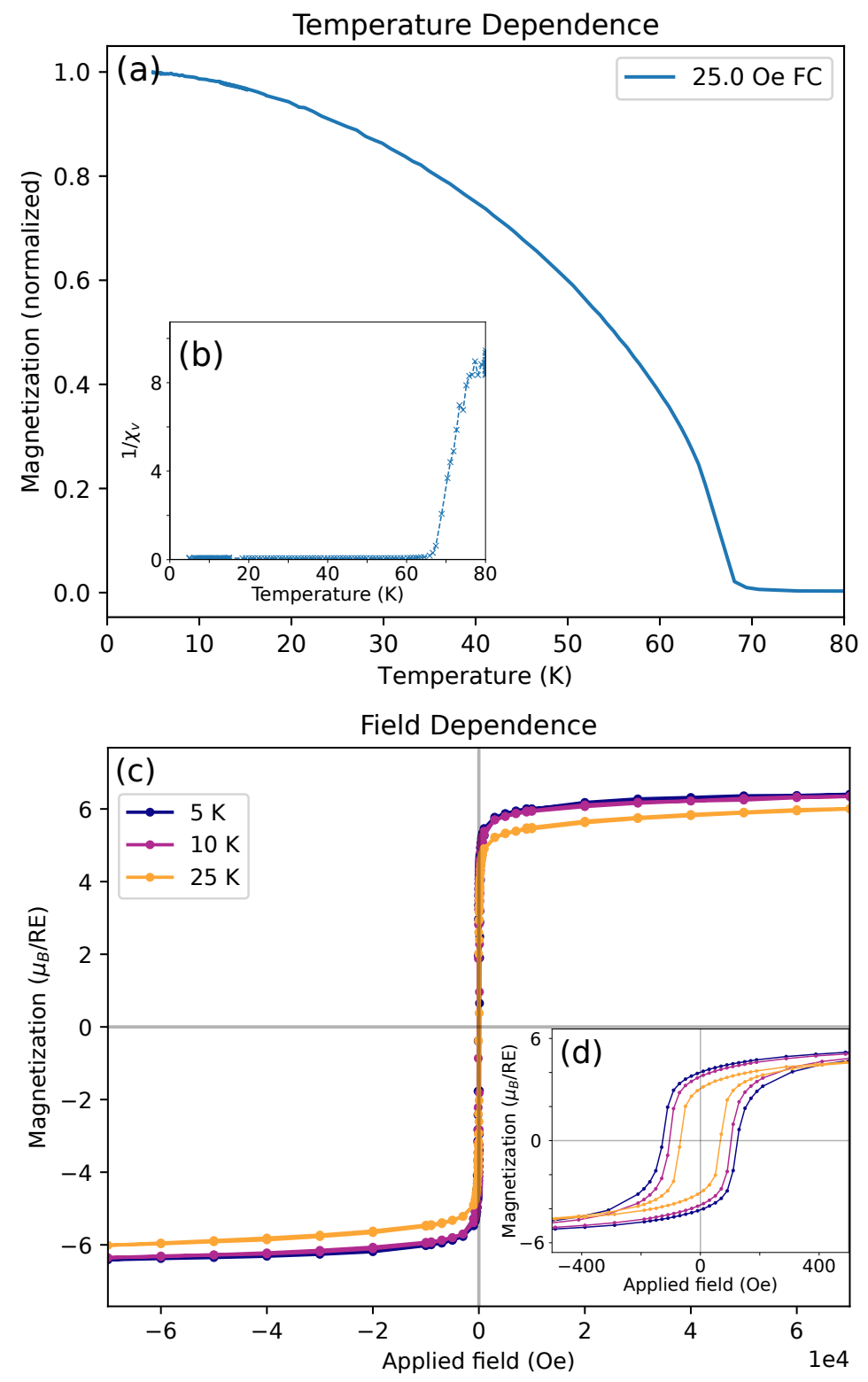

Figure 6.16: Temperature and field dependent magnetic measurements of an epitaxial GdN sample capped with Sm after undergoing an annealing process to thermally desorb the Sm. (a) Field cooled curve using an applied field of 25 Oe and (b) corresponding inverse susceptibility showing a ferromagnetic transition with $T_{C}=67 \mathrm{~K}$. (c) Field dependence at different temperatures showing magnetic hysteresis and magnetic saturation of $\sim 6 \mu_{\mathrm{B}} / \mathrm{Gd}^{3+}$. (d) Zoomed inset of hysteresis close to zero field showing low coercivity of 130 Oe at $5 \mathrm{~K}$. 

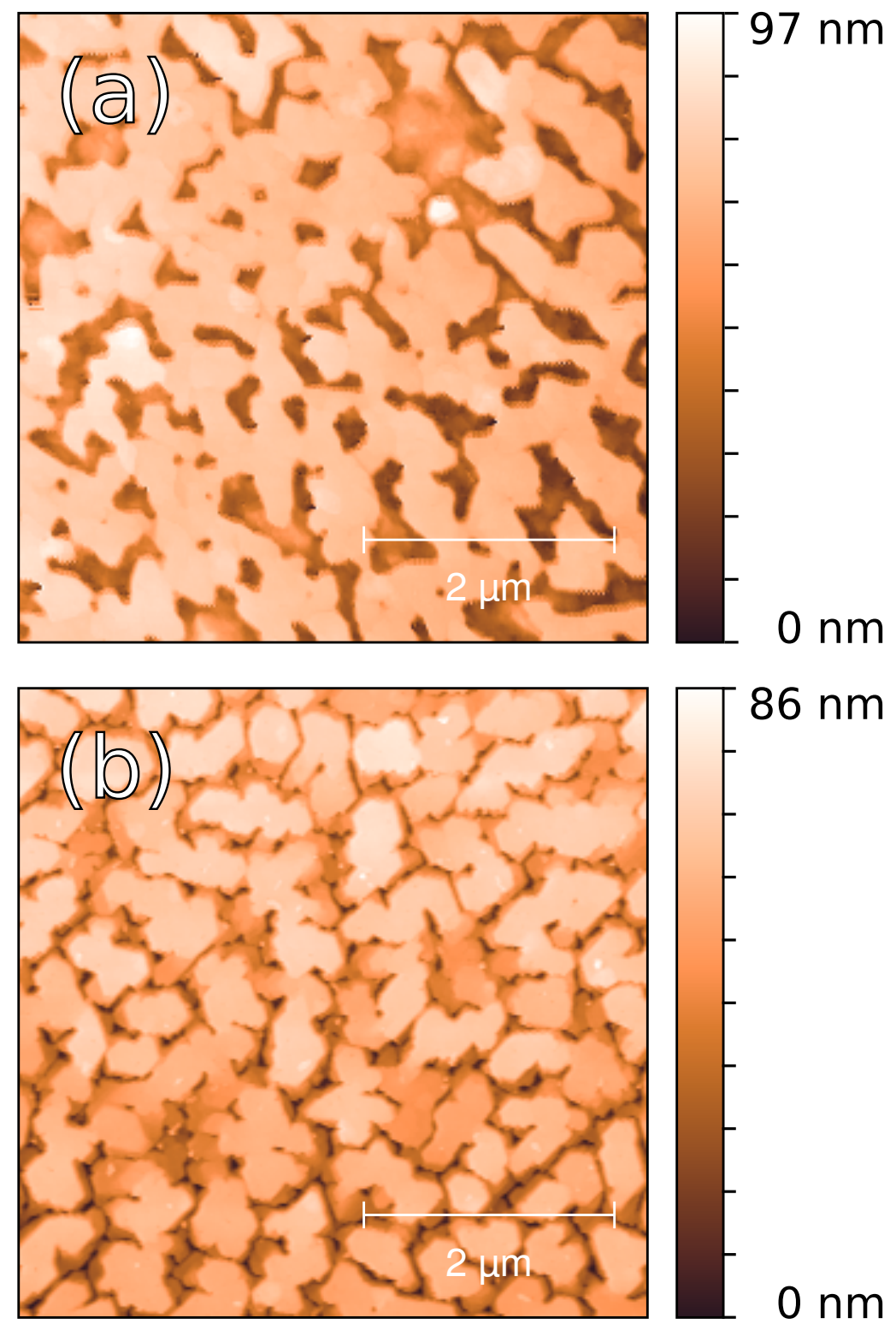

Figure 6.17: AFM images of two different Sm-capped GdN samples. The GdN/Sm layer thicknesses are (a) $100 \mathrm{~nm} / 100 \mathrm{~nm}$ and (b) $70 \mathrm{~nm} 50 \mathrm{~nm}$. Pits across the surface are assumed to result from oxidation of the Sm capping layer. 


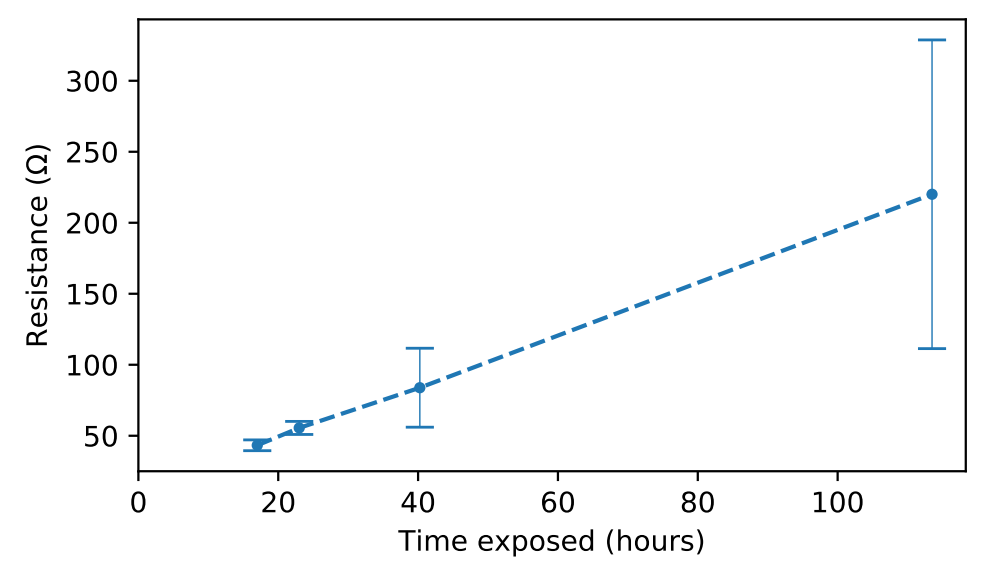

Figure 6.18: Two point resistance of a Sm capped GdN sample showing a linear increase with time as it is left exposed to air. After about 5 days, the resistance can no longer be measured and the film undergoes delamination after a couple of weeks.

\subsubsection{Sm capping layer exposed to atmosphere}

This work was carried out in collaboration with Maxime Le Ster, a PhD student in the group of Prof. Simon Brown at Canterbury University, Christchurch, in order to investigate the surface of GdN using scanning tunnelling microscopy after removing the Sm capping layer.

As noted in Section 6.2.2, the Sm capping layer is expected to oxidise on exposure to the atmosphere, likely changing its properties. By measuring the electrical resistance of the Sm film left in ambient atmosphere as a function of time, the effect of oxidation can be seen. This measurement, performed by Le Ster, show that the two point resistance increases linearly with time while a sample stored under vacuum desiccation shows no change over time (Fig. 6.18). After a couple of weeks in ambient atmosphere, the Sm film undergoes delamination from the substrate as well.

We have investigated this using AFM measurements of the surfaces of different Sm-capped samples which show how the properties and quality of 
the underlying GdN layer can influence the resulting Sm capping layer quality and the differing degrees to which oxidation can affect the morphology of the capping layer.

AFM images performed in ambient conditions of two different Sm-capped epitaxial GdN samples are shown in Fig. 6.17 with different Sm capping thicknesses of (a) $100 \mathrm{~nm}$ and (b) $50 \mathrm{~nm}$. Note that these samples were also grown using different AlN templates which may have varying quality. These images, taken after the samples were exposed to atmosphere for only a few hours, show significant differences in the initial state of the Sm capping layer and progression of oxidation for the two samples. The capping layer shown in Fig. 6.17a shows oxidation starting from a contiguous capping layer while Fig. 6.17b has a clearer granular structure visible in the Sm film. Another difference between the two samples shown is the thickness of the GdN layer ((a) $100 \mathrm{~nm}$ and (b) $70 \mathrm{~nm}$ ) likely resulting in different GdN surface quality upon which the $\mathrm{Sm}$ is then deposited. Sample (a) has a GdN $\langle 111\rangle$ rocking curve FWHM of $1.47^{\circ}$ compared to a FWHM of sample (b) of $0.78^{\circ}$. Further work is required to identify the best thickness for the removable Sm capping layer while considering the removal process that is to be used.

As a start and to limit the effect of oxidation, a single Sm-capped epitaxial $\mathrm{GdN}$ sample was cleaved into two pieces. One piece was stored under vacuum for later ex situ measurements while the other was reloaded into the UHV chamber after having been exposed to air for fewer than 10 minutes. The respective GdN and Sm thicknesses were $30 \mathrm{~nm}$ and $80 \mathrm{~nm}$. After reloading the sample, it was noted that the same RHEED pattern that was present directly after growth of the Sm capping layer could not be reobtained and only a diffuse RHEED image was seen. Once this piece was heated at around $200{ }^{\circ} \mathrm{C}$ for 15 to 30 minutes, it was possible to recover diffraction patterns of Sm similar to those seen in Fig. 6.12. The sample was then heated for about 45 minutes at a temperature of $500{ }^{\circ} \mathrm{C}$ to $550{ }^{\circ} \mathrm{C}$. The double spot pattern characteristic of GdN was clearly identifiable suggesting that the majority of the Sm cap was thermally desorbed. It is important to note however, as 


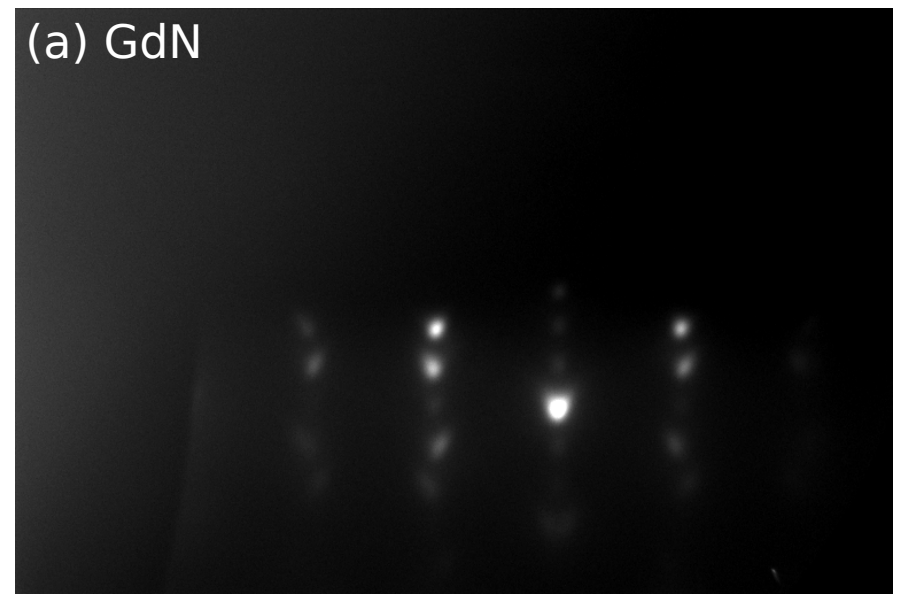

\section{(b) Sm}

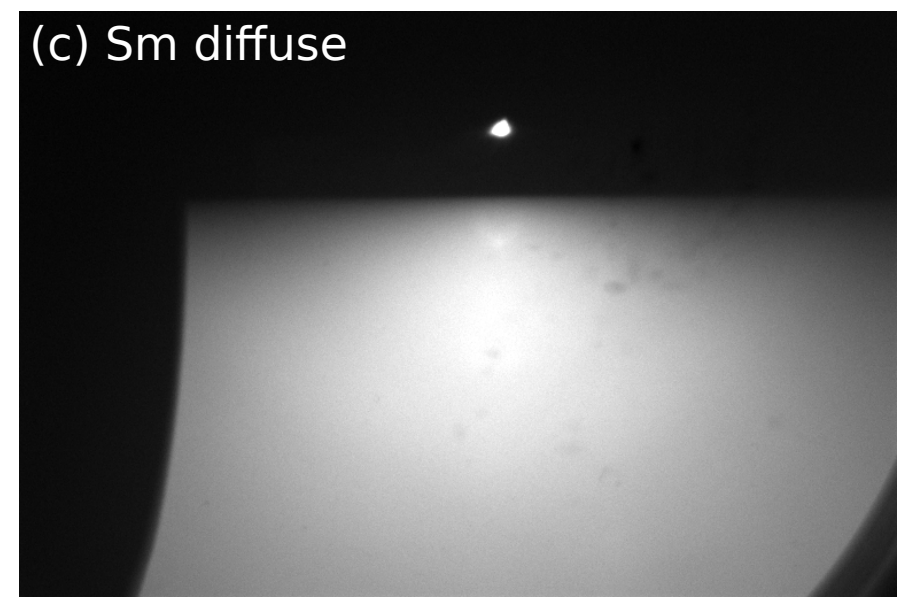

Figure 6.19: RHEED images showing (a) epitaxial GdN, capped with (b) Sm and (c) the diffuse pattern that is observed after the Sm-capped sample is exposed to atmosphere for several minutes. 
discussed below, that the time and temperature of the annealing are critical and that slight variations can lead to the formation of ring diffraction lines that could suggest deterioration of the GdN surface.

To investigate this more systematically, a Sm-capped GdN sample was grown on a full 2 inch AlN/Si template, and cleaved pieces of this sample were annealed at varying temperatures to understand the desorption of partially oxidised Sm.

RHEED images taken of this sample are shown in Fig. 6.19. The estimated film thicknesses are $80 \mathrm{~nm}$ of GdN and $50 \mathrm{~nm}$ of Sm. Fig. 6.19a shows the epitaxial GdN (111) surface with rotational twinning as before. While the epitaxial $\mathrm{Sm}$ is visible in the RHEED immediately after growth (Fig. 6.19b), once the sample is removed from the chamber and cleaved for further systematic study, the same RHEED pattern cannot be reobtained after a piece is reintroduced into the chamber as previously mentioned. Only a diffuse RHEED image is seen (Fig. 6.19k) after total exposure to air on the order of minutes. This suggests that even for this short exposure time, the oxide thickness is likely already around a few nm to block the RHEED probing of crystalline Sm beneath.

Once these pieces are back in the chamber and the heating process is started, it is possible to recover diffraction patterns similar to those seen in Fig. 6.12. RHEED patterns taken after annealing at fixed heater currents for 30 minutes are shown in Fig. 6.20. At the lower heater currents used, we do not expect the entire capping layer to be thermally desorbed (Section 6.2.3 but we do see the Sm diffraction pattern slowly recovered. At the higher currents used, $4.2 \mathrm{~A}\left(\sim 620^{\circ} \mathrm{C}\right)$ and $4.5 \mathrm{~A}\left(\sim 650^{\circ} \mathrm{C}\right)$, the double spot pattern characteristic of $\mathrm{GdN}$ is clearly identified, but pronounced ring diffraction lines begin to form. Compared to the annealing of unexposed Sm (Fig. 6.12), the resulting surface is considerably degraded. Although the majority of the Sm cap is thermally desorbed, the $\mathrm{Sm}_{2} \mathrm{O}_{3}$ formed during exposure to atmosphere likely migrates down through the film during the annealing process to react with the GdN layer, causing the polycrystalline 

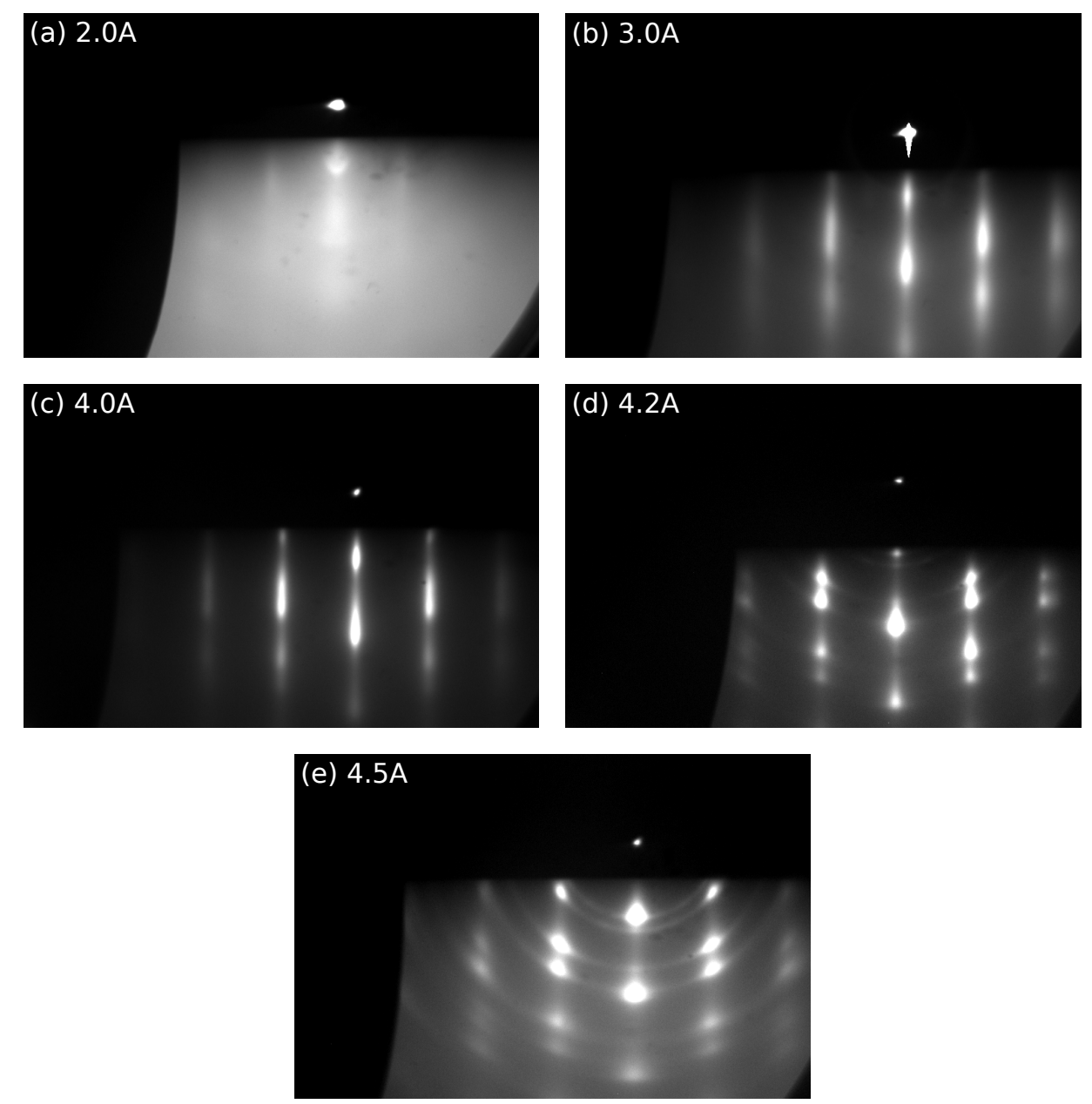

Figure 6.20: RHEED images taken after annealing different pieces of a Sm-capped GdN sample for 30 minutes at (a) $2.0 \mathrm{~A}\left(\sim 400^{\circ} \mathrm{C}\right)$, (b) $3.0 \mathrm{~A}$ $\left(\sim 500^{\circ} \mathrm{C}\right)(\mathrm{c}) 4.0 \mathrm{~A}\left(\sim 600^{\circ} \mathrm{C}\right),(\mathrm{d}) 4.2 \mathrm{~A}\left(\sim 620^{\circ} \mathrm{C}\right),(\mathrm{e}) 4.5 \mathrm{~A}\left(\sim 650^{\circ} \mathrm{C}\right)$. A progression is seen where the thermal desorption of $\mathrm{Sm}$ continues further at higher heater currents until the GdN pattern is recovered above $4.2 \mathrm{~A}$ in 30 minutes. The highest heater currents used result in a degraded GdN surface as evidenced by significant polycrystalline arcs in the RHEED pattern. 
rings to form.

These sample pieces were removed from the chamber without depositing any additional capping layer for further measurement. XRD scans are shown in Fig. 6.21. Notably, despite the double spot pattern of GdN being observed in the RHEED for the 4.2 A annealed sample, the XRD scan indicates that a significant amount of Sm remains, and only the $4.5 \mathrm{~A}$ anneal removed $\mathrm{Sm}$ almost entirely, resulting in the oxidation of the unprotected GdN layer.

\subsection{Discussion and conclusion}

We have investigated the viability of using Sm as a removable capping layer material for REN thin film samples. The main outstanding issues preventing the successful removal for experimental measurements of the REN film surface are the effects of residual samarium oxide which is not fully removed during the annealing process attempted, and inconclusive evidence for whether the annealing process does in fact remove the entirety of the Sm even without the presence of oxide.

\subsubsection{Samarium oxide}

The formation of samarium oxide is an unavoidable issue if the sample is to be transferred between experimental chambers. The two samples shown in section 6.6 .2 show that the state of the Sm capping layer can differ in many ways depending on the entire sample growth process. It may be possible to optimise the deposition process of the Sm layer to decrease the amount of oxide that can form.

Another option to consider is an additional step in the decapping process to remove the surface oxide and other possible surface contaminants such an ion sputtering process. Such a process has been implemented successfully for the case of $\mathrm{Bi}_{2} \mathrm{Se}_{3}$ capped with amorphous $\mathrm{Se}[92$ in which an $\mathrm{Ar}$ ion sputtering was carried out before the thermal annealing to remove surface 


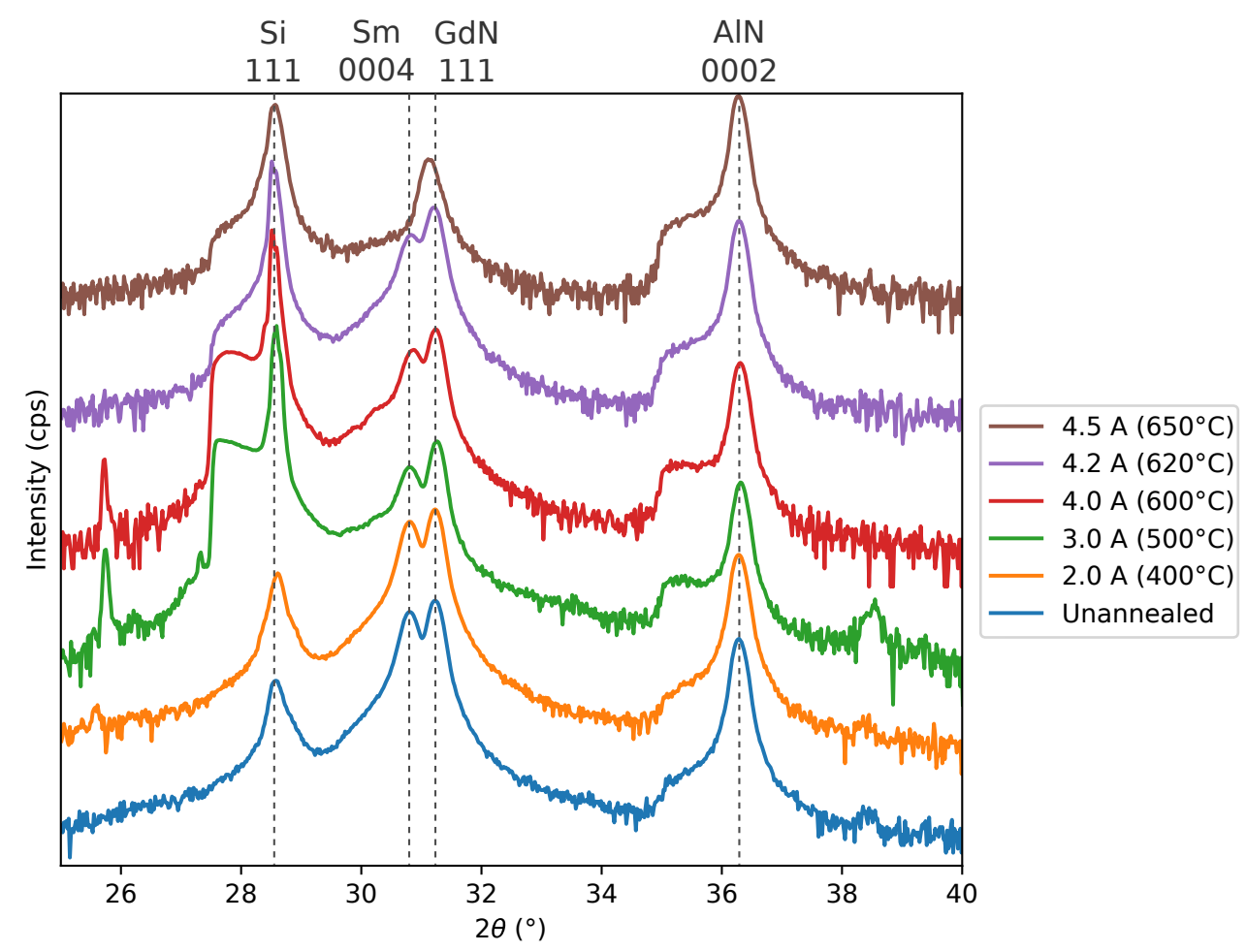

Figure 6.21: XRD 2 $\theta-\omega$ scans of a Sm-capped GdN sample annealed at fixed heater currents for 30 minutes. No capping layer was deposited after the anneal. At $4.5 \mathrm{~A}\left(\sim 650^{\circ} \mathrm{C}\right)$, the Sm appears to have been fully removed, resulting in the oxidation of the GdN layer beneath. The GdN/Sm "merges" into a single peak in the $4.5 \mathrm{~A}$ scan, with a significantly smaller peak area. 
(a)
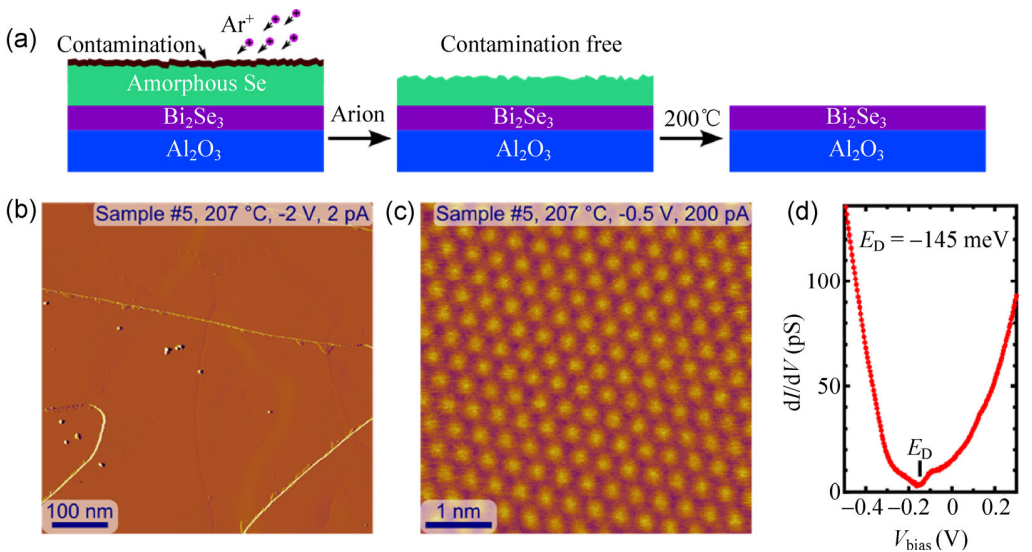

Figure 6.22: Details of the decapping process of amorphous Se. (a) An Ar ion sputtering is performed to remove contamination from the Se surface before a mild anneal is used to reveal the pristine $\mathrm{Bi}_{2} \mathrm{Se}_{3}$ surface. (b, c) AFM images showing the clean recovered surface and atomic resolution of the Se surface lattice. Reprinted by permission from Springer Nature from [92], (C)2014

contaminants which could not be removed thermally (Fig.6.22). Preliminary results obtained by Le Ster for combining sputtering and thermal annealing of the Sm capping layer have been promising although a big challenge is identifying the optimum sputtering conditions for the Sm cap system.

\subsubsection{Annealing time}

Finding the optimum annealing time for a chosen annealing temperature is necessary to limit the effect of thermally desorbing partially oxidised Sm. As seen in the fixed annealing time of 30 minutes at various heater currents in section 6.6.2, the highest temperature anneals resulted in a degraded final surface. Further investigation is required to identify whether a longer anneal at a lower temperature or a shorter anneal at high temperature is more suitable for recovering the best GdN surface, and whether or not this depends also on the degree to which the Sm cap has oxidised. As the results in section 
6.6.1 suggest that GdN as-grown is largely unaffected by annealing at the highest temperatures used for thermally desorbing the Sm capping layers, the optimum annealing recipe should aim to reduce migration of whatever contaminants remain on the surface to the GdN layer during annealing.

\subsubsection{Conclusion}

The viability of using $\mathrm{Sm}$ as a removable capping layer material for REN thin film samples has been investigated. Sm is shown to work as a passivating layer for GdN on a short-term scale, allowing measurements on the GdN layer such as XRD and SQUID magnetometry. Sm itself exhibits some interesting properties when grown on top of epitaxial GdN films such as the fact that it grows epitaxially at room temperature, smooth enough to exhibit surface reconstructions. Its reactivity, however, introduces some issues when trying to thermally desorb the capping layer as it seems $\mathrm{Sm}_{2} \mathrm{O}_{3}$ remains to react detrimentally with the GdN layer. Removal of surface contamination through an additional step such as Ar ion sputtering is suggested as a possible solution currently under investigation. 


\section{Chapter 7}

\section{Summary and outlook}

\section{$7.1 \quad$ Summary}

In this thesis, the growth and structural characterisation of thin films of GdN and $\mathrm{SmN}$, two members of the rare earth nitride class of materials, has been explored. The structural properties are related to the electrical and magnetic properties of the films.

The formation of a preferential orientation in polycrystalline GdN thin films was investigated primarily using x-ray diffraction on thin films of varying thickness grown on amorphous fused silica substrates. GdN is observed to grow preferentially along the [1 11 ] crystal direction perpendicular to the substrate, and this process is understood in terms of an evolutionary selection process selecting for crystallites oriented with a fast growth axis oriented nearly parallel to the film growth direction. The influence of the variations in the deposition parameters between film growths are correlated to the film properties. Most notably, a wide range of average lattice constants are observed across samples along with a huge range of film conductivities ranging from heavily doped semiconducting behaviour to highly insulating films. Magnetic behaviour of all of the GdN thin films was consistent with previous observations of GdN magnetism in polycrystalline films; Curie temperatures ranging between $60 \mathrm{~K}$ to $75 \mathrm{~K}$ with coercive fields between 200 Oe to 500 Oe. 
The epitaxial growth of $\mathrm{SmN}$ on AlN surfaces was studied as a function of growth temperature. A novel growth orientation transition was observed, from the previously known and expected (111)-oriented SmN on AlN 0001 to an unexpected (001)-oriented $\mathrm{SmN}$ on the same surface at high growth temperatures $\left(\geq 800^{\circ} \mathrm{C}\right)$. This observation is confirmed using ex situ x-ray diffraction and in situ RHEED. A computational simulation tool was developed to model and understand the electron diffraction pattern resulting from the new epitaxial orientation. The first direct measurements of the $\mathrm{SmN}$ surface morphology via STM were also obtained. The origin of the growth orientation transition and its dependence on the nitrogen precursor used are discussed.

The growth of Sm layers on REN thin film samples has been studied with the goal of developing a temporary capping layer solution. Sm is the second most volatile rare earth element and the possibility of removing deposited Sm layers thermally is explored. Sm is noted to grow epitaxially on top of epitaxially grown GdN at room temperature, observed through ex situ x-ray diffraction and in situ RHEED, with surface reconstructions visible in the latter attesting to the quality of the as-deposited layer. However, reactivity of Sm with the atmosphere when removed from the growth chamber introduce problems with the thermal removal of the capping layer.

\section{$7.2 \quad$ Future work}

Many limitations exist in the studies presented in this thesis. While the effect of variation in the growth parameters was probed for polycrystalline $\mathrm{GdN}$, a systematic investigation into wider ranges of Gd and $\mathrm{N}_{2}$ fluxes would undoubtedly reveal much more about the film growth process. High temperature polycrystalline deposition as well as post-deposition annealing are also two related topics that have not been explored for REN thin films. Replicating these studies for other REN members is another avenue of research.

The main tool for the GdN film microstructure characterisation was x- 
ray diffraction, however the hardware setup available did not allow for complicated asymmetric measurements to be made. In particular, pole figure measurements that probe a wide area of reciprocal space would be an ideal measurement to make to fully determine the crystallographic texture of the film beyond just the preferred growth orientation. The ability to make measurements like this may be very helpful in characterising future REN film samples.

The growth orientation transition observed for epitaxial SmN on AlN surfaces is interesting for a number of reasons. The question remains whether any other REN members could also exhibit a similar orientation transition, although the low volatility of $\mathrm{Sm}$ in particular has been hypothesised to be a major factor. The $\left(\begin{array}{lll}0 & 0 & 1\end{array}\right)$ oriented $\mathrm{SmN}$ could potentially be used as a buffer layer to grow other RENs in a $(001)$ orientation without requiring cubic substrates, possibly making a test bed for orientation-dependent properties of REN thin films.

A complete decapping process for removable Sm capping layers has not yet been achieved due to the effects of residual samarium oxide on the annealing process. Work has started on an in situ ion bombardment of the capping layer prior to a thermal annealing to try to eliminate the residual samarium oxide effects with some success, but further refinement is still required. A successful process would allow characterisation of REN surfaces by techniques that have previously been impossible through standard capping layers such as angle-resolved photoemission spectroscopy. 


\section{Bibliography}

[1] C. Chappert, A. Fert, and F. N. Van Dau. "The emergence of spin electronics in data storage". Nature materials 6.11 (2007), p. 813. DOI: $10.1038 /$ nmat2024.

[2] S. Wolf et al. "Spintronics: a spin-based electronics vision for the future". Science 294.5546 (2001), pp. 1488-1495. DOI: 10 . 1126 / science.1065389.

[3] I. Žutić, J. Fabian, and S. D. Sarma. "Spintronics: Fundamentals and applications". Reviews of Modern Physics 76.2 (2004), p. 323. DOI: 10.1103/RevModPhys.76.323.

[4] D. D. Awschalom and M. E. Flatté. "Challenges for semiconductor spintronics". Nature physics 3.3 (2007), p. 153. DOI: $10.1038 /$ nphys551.

[5] C. Aerts et al. "Half-metallic to insulating behavior of rare-earth nitrides". Physical Review B 69.4 (2004), p. 045115. DOI: 10 . $1103 /$ PhysRevB.69.045115.

[6] P. Wachter and E. Kaldis. "Magnetic interaction and carrier concentration in GdN and $\mathrm{GdN}_{1-x} \mathrm{O}_{x}$ ". Solid State Communications 34.4 (1980), pp. 241-244. DOI: 10.1016/0038-1098(80)90401-9.

[7] F. Natali et al. "Rare-earth mononitrides". Progress in Materials Science 58.8 (2013), pp. 1316-1360. DOI: 10.1016/j .pmatsci.2013.06. 002 . 
[8] E.-M. Anton et al. "NdN: An intrinsic ferromagnetic semiconductor". Physical Review B 93.6 (2016), p. 064431. DoI: 10.1103/PhysRevB. 93.064431.

[9] E.-M. Anton et al. "Spin/orbit moment imbalance in the near-zero moment ferromagnetic semiconductor SmN". Physical Review B 87.13 (2013), p. 134414. DOI: 10.1103/physrevb.87.134414.

[10] D. Li et al. "Magnetic properties of stoichiometric Gd monopnictides". Journal of Physics: Condensed Matter 9.48 (1997), p. 10777. DOI: 10. 1088/0953-8984/9/48/019.

[11] F. Ullstad et al. "Ohmic contacts of Au and Ag metals to n-type GdN thin films". AIMS Materials Science 2.2 (2015), pp. 79-85. DOI: 10 . 3934/matersci.2015.2.79.

[12] J. R. Chan et al. "Temperature-Induced Four-Fold-on-Six-Fold Symmetric Heteroepitaxy, Rocksalt SmN on Hexagonal AlN". Crystal Growth $\&$ Design 16.11 (2016), pp. 6454-6460. DOI: 10.1021/acs.cgd.6b01133.

[13] J. R. Chan et al. "Effect of the growth temperature and nitrogen precursor on the structural and electrical transport properties of $\mathrm{SmN}$ thin films". MRS Advances 2.3 (2017), pp. 165-171. DOI: $10.1557 /$ $\operatorname{adv} .2017 .74$.

[14] F. Natali et al. "Rare Earth Nitride Structures and Devices and Method for Removing a Passivating Capping". European Patent 16202663.7 (Filed 07 December 2016).

[15] F. Natali et al. "Rare Earth Nitride Structure or Device and Fabrication Method". US Patent No. 15/607,596 (Filed 29 May 2017).

[16] F. Hulliger. "Magnetic properties of the rare earth pnictides". Journal of Magnetism and Magnetic Materials 8.3 (1978), pp. 183-205. DOI: 10.1016/0304-8853(78)90121-X. 
[17] R. Brown and N. Clark. "Composition limits and vaporization behaviour of rare earth nitrides". Journal of Inorganic and Nuclear Chemistry 36.11 (1974), pp. 2507-2514. DOI: 10 . 1016/0022-1902(74) 80462-8.

[18] R. A. Cutler and A. Lawson. "Synthesis and magnetic behavior of GdN". Journal of Applied Physics 46.6 (1975), pp. 2739-2744. DOI: 10.1063/1.321949.

[19] N. Plank et al. "Enhanced Curie temperature in N-deficient GdN". Applied Physics Letters 98.11 (2011), p. 112503. DoI: $10.1063 / 1$. 3566996.

[20] O. Vogt and K. Mattenberger. "Magnetic measurements on rare earth and actinide monopnictides and monochalcogenides". Handbook on the Physics and Chemistry of Rare Earths 17 (1993), pp. 301-407. DOI: 10.1016/S0168-1273(05)80031-5.

[21] D. Le Binh et al. "Europium Nitride: a novel diluted magnetic semiconductor". Physical review letters 111.16 (2013), p. 167206. DOI: 10. 1103/PhysRevLett.111.167206.

[22] F. Natali et al. "Role of magnetic polarons in ferromagnetic GdN". Physical Review B 87.3 (2013), p. 035202. DOI: 10.1103/PhysRevB. 87.035202 .

[23] S. Granville et al. "Semiconducting ground state of GdN thin films". Physical Review B 73.23 (2006), p. 235335. DOI: 10.1103/PhysRevB. 73.235335.

[24] F. Natali et al. "Epitaxial growth of GdN on silicon substrate using an AlN buffer layer". Journal of Crystal Growth 312.24 (2010), pp. 35833587. DOI: $10.1016 / \mathrm{j} \cdot$ jcrysgro.2010.09.030.

[25] F. Natali et al. "Epitaxial growth and properties of GdN, EuN and SmN thin films". Physica Status Solidi C: Conferences and Critical Reviews 9.3-4 (2012), pp. 605-608. DOI: 10.1002/pssc. 201100363. 
[26] C. M. Lee. "Electrical and Magnetic Investigations of Magnesiumdoped Epitaxial Gadolinium Nitride Thin Films". Victoria University of Wellington (2015). DOI: 10063/4764.

[27] H. Trodahl et al. "Ferromagnetic redshift of the optical gap in GdN". Physical Review B 76.8 (2007), p. 085211. DoI: 10.1103/PhysRevB. 76.085211 .

[28] H. Yoshitomi et al. "Optical and magnetic properties in epitaxial GdN thin films". Physical Review B 83.15 (2011), p. 155202. DOI: 10.1103/ PhysRevB.83.155202.

[29] M. Azeem et al. "Optical response of DyN". Journal of Applied Physics 113.20 (2013), p. 203509. DOI: 10.1063/1.4807647.

[30] J. Richter et al. "Electronic structure of EuN: Growth, spectroscopy, and theory". Physical Review B 84.23 (2011), p. 235120. DOI: 10.1103/ PhysRevB.84.235120.

[31] H. Warring et al. "YbN: An intrinsic semiconductor with antiferromagnetic exchange". Physical Review B 90.24 (2014), p. 245206. DoI: 10.1103/PhysRevB.90.245206.

[32] T.-Y. Lee et al. "Growth and physical properties of epitaxial CeN layers on MgO (001)". Journal of Applied Physics 94.2 (2003), pp. 921-927. DOI: $10.1063 / 1.1579113$

[33] J. Gerlach, J. Mennig, and B. Rauschenbach. "Epitaxial gadolinium nitride thin films". Applied Physics Letters 90.6 (2007), p. 061919. DOI: $10.1063 / 1.2472538$

[34] M. Scarpulla et al. "GdN (111) heteroepitaxy on GaN (0001) by $\mathrm{N}_{2}$ plasma and $\mathrm{NH}_{3}$ molecular beam epitaxy". Journal of Crystal Growth 311.5 (2009), pp. 1239-1244. DOI: 10.1016/j . jcrysgro. 2008.12.050.

[35] B. Ludbrook et al. "Growth and properties of epitaxial GdN". Journal of Applied Physics 106.6 (2009), p. 063910. Dor: 10.1063/1.3211290. 
[36] F. Natali et al. "Molecular beam epitaxy of ferromagnetic epitaxial GdN thin film". Journal of Crystal Growth 404 (2014), pp. 146-151. DOI: $10.1016 / j \cdot j$ jcrysgro.2014.07.010.

[37] S. Vézian et al. "AlN interlayer to improve the epitaxial growth of $\mathrm{SmN}$ on GaN (0001)". Journal of Crystal Growth 450 (2016), pp. 22-27. DOI: $10.1016 / j \cdot j$ crysgro.2016.06.006

[38] A. P. Milanov et al. "Homoleptic Gadolinium Guanidinate: A Single Source Precursor for Metal-Organic Chemical Vapor Deposition of Gadolinium Nitride Thin Films". Journal of the American Chemical Society 131.47 (2009), pp. 17062-17063. DOI: 10.1021/ja907952g.

[39] T. B. Thiede et al. "Evaluation of homoleptic guanidinate and amidinate complexes of gadolinium and dysprosium for MOCVD of rareearth nitride thin films". Chemistry of Materials 23.6 (2011), pp. 14301440. DOI: $10.1021 / \mathrm{cm} 102840 \mathrm{v}$.

[40] O. Ambacher. "Growth and applications of group III-nitrides". Journal of Physics D: Applied Physics 31.20 (1998), p. 2653. Dor: 10.1088/ 0022-3727/31/20/001.

[41] J. Chan. Kinematic RHEED simulation implemented in python. 2017. URL: https://github.com/chanjr/rheedsim

[42] N. Nagaosa et al. "Anomalous hall effect". Reviews of modern physics 82.2 (2010), p. 1539. DOI: 10.1103/RevModPhys.82.1539.

[43] A. H. Warring. "Device Applications of Rare-Earth Nitrides". Victoria University of Wellington (2016). DOI: 10063/5452.

[44] L. Schulz. "The structure and growth of evaporation $\mathrm{LiF}$ and $\mathrm{NaCl}$ films on amorphous substrates". The Journal of Chemical Physics 17.11 (1949), pp. 1153-1162. DOI: 10.1063/1.1747131.

[45] H. K. Yu and J.-L. Lee. "Understanding of Preferred Orientation Formation in Rock-Salt Materials: The Case of MgO". Crystal Growth \&3 Design 16.4 (2016), pp. 1978-1983. DOI: 10.1021/acs.cgd.5b01564. 
[46] A. Van der Drift. "Evolutionary selection, a principle governing growth orientation in vapour-deposited layers". Philips Research Reports 22.3 (1967), pp. 267-88.

[47] B. Movchan and A. Demchishin. "STRUCTURE AND PROPERTIES OF THICK CONDENSATES OF NICKEL, TITANIUM, TUNGSTEN, ALUMINUM OXIDES, AND ZIRCONIUM DIOXIDE IN VACUUM." Fizika metallov $i$ metallovedenie 28.653 (Oct. 1969), p. 60 .

[48] J. A. Thornton. "The microstructure of sputter-deposited coatings". Journal of Vacuum Science \& Technology A: Vacuum, Surfaces, and Films 4.6 (1986), pp. 3059-3065. DOI: 10.1116/1.573628.

[49] R. Messier, A. Giri, and R. Roy. "Revised structure zone model for thin film physical structure". Journal of Vacuum Science \&f Technology A: Vacuum, Surfaces, and Films 2.2 (1984), pp. 500-503. DOI: 10.1116/ 1.572604

[50] E. Mirica, G. Kowach, and H. Du. "Modified structure zone model to describe the morphological evolution of $\mathrm{ZnO}$ thin films deposited by reactive sputtering". Crystal Growth \& Design 4.1 (2004), pp. 157-159. DOI: $10.1021 / \mathrm{cg} 025596 \mathrm{~b}$.

[51] I. Petrov et al. "Microstructural evolution during film growth". Journal of Vacuum Science \& Technology A: Vacuum, Surfaces, and Films 21.5 (2003), S117-S128. DOI: 10.1116/1.1601610.

[52] F. Hulliger. "Rare earth pnictides". Handbook on the physics and chemistry of rare earths 4 (1979), pp. 153-236. DOI: 10 . 1016/S01681273(79) 04006-X.

[53] K. Senapati et al. "Structural evolution and competing magnetic orders in polycrystalline GdN films". Physical Review B 83.1 (2011), p. 014403. DOI: 10.1103/PhysRevB.83.014403. 
[54] E. Chason. "A kinetic analysis of residual stress evolution in polycrystalline thin films". Thin Solid Films 526 (2012), pp. 1-14. DOI: $10.1063 / 1.4704683$

[55] P. Scardi, M. Leoni, and R. Delhez. "Line broadening analysis using integral breadth methods: a critical review". Journal of Applied Crystallography 37.3 (2004), pp. 381-390. DOI: 10.1107/S0021889804004583

[56] R. Gambino et al. "Magnetic Properties and Structure of GdN and GdN1- x O x". Journal of Applied Physics 41.3 (1970), pp. 933-934. DOI: $10.1063 / 1.1659030$

[57] S. Ingvarsson et al. "Thickness-dependent magnetic properties of Ni81Fe19, Co90Fe10 and Ni65Fe15Co20 thin films". Journal of magnetism and magnetic materials 251.2 (2002), pp. 202-206. DOI: 10.1016/S0304-8853(02) 00577-2

[58] E. Kneller and F. Luborsky. "Particle size dependence of coercivity and remanence of single-domain particles". Journal of Applied Physics 34.3 (1963), pp. 656-658. DOI: 10.1063/1.1729324.

[59] M. Knobel et al. "Superparamagnetism and other magnetic features in granular materials: a review on ideal and real systems". Journal of Nanoscience and Nanotechnology 8.6 (2008), pp. 2836-2857. DOI: 10.1166/jnn.2008.15348.

[60] E. Wohlfarth. "A mechanism of magnetic hysteresis in heterogeneous alloys". Philosophical Transactions of the Royal Society A 240.826 (1948), pp. 599-642. DOI: 10.1098/rsta.1948.0007.

[61] C.-M. Lee et al. "Highly resistive epitaxial Mg-doped GdN thin films". Applied Physics Letters 106.2 (2015), p. 022401. DOI: 10.1063/1. 4905598.

[62] C. Meyer et al. "Near-zero-moment ferromagnetism in the semiconductor SmN". Physical Review B 78.17 (2008), p. 174406. Dor: 10.1103/ PhysRevB.78.174406. 
[63] E.-M. Anton et al. "Superconductivity in the ferromagnetic semiconductor samarium nitride". Physical Review B 94.2 (2016), p. 024106. DOI: $10.1103 /$ PhysRevB.94.024106.

[64] J. Linder and J. W. Robinson. "Superconducting spintronics". Nature Physics 11.4 (2015), pp. 307-315. DOI: 10.1038/nphys3242.

[65] A. Le Louarn et al. "AlN buffer layer growth for GaN epitaxy on (111) Si: Al or N first?" Journal of Crystal Growth 311.12 (2009), pp. 32783284. DOI: $10.1016 / \mathrm{j}$. jcrysgro.2009.04.001.

[66] C. Lee et al. "Reconstructions of the AlN (0001) surface". Physical Review B 68.20 (2003), p. 205317. DOI: 10.1103/PhysRevB.68.205317.

[67] K. H. Ahn, S. Baik, and S. S. Kim. "Change of growth orientation in Pt films epitaxially grown on $\mathrm{MgO}$ (001) substrates by sputtering". Journal of Materials Research 17.09 (2002), pp. 2334-2338. DOI: 10 . 1557/jmr.2002.0342.

[68] B. Xiao et al. "Competition between (001) and (111) $\mathrm{MgO}$ thin film growth on $\mathrm{Al}$-doped $\mathrm{ZnO}$ by oxygen plasma assisted pulsed laser deposition". Journal of Applied Physics 113.21 (2013), p. 214102. DOI: $10.1063 / 1.4807933$.

[69] Y. Kato et al. "Fabrication of semiconducting $\mathrm{SrB}_{6-\delta}$ thin films on ultrasmooth sapphire substrates by laser molecular beam epitaxy". Journal of Crystal Growth 312.3 (2010), pp. 378-381. DOI: $10.1016 / \mathrm{j}$. jcrysgro.2009.11.015.

[70] M. Grundmann, T. Böntgen, and M. Lorenz. "Occurrence of Rotation Domains in Heteroepitaxy". Physical Review Letters 105.14 (2010), p. 146102. DOI: 10.1103/PhysRevLett.105.146102.

[71] M. Grundmann. "Formation of epitaxial domains: Unified theory and survey of experimental results". Physica Status Solidi B: Basic Solid State Physics 248.4 (2011), pp. 805-824. DOI: 10.1002/pssb. 201046530 . 
[72] N. Tarakina et al. "Suppressing twin formation in $\mathrm{Bi}_{2} \mathrm{Se}_{3}$ thin films". Advanced Materials Interfaces 1.5 (2014). DOI: 10 . 1002 / admi . 201400134.

[73] W. Luo et al. "Epitaxial growth of $\mathrm{SrTiO}_{3}\left(\begin{array}{lll}0 & 0 & 1\end{array}\right)$ films on multilayer buffered GaN (lo 000 2) by pulsed laser deposition". Journal of Physics D: Applied Physics 46.6 (2013), p. 065307. Dor: 10.1088/0022-3727/ 46/6/065307.

[74] J. Griesche, R. Enderlein, and D. Schikora. "Orientation and strain in heteroepitaxial growth". Physica Status Solidi A: Applications and Materials Science 109.1 (1988), pp. 11-38. DOI: 10.1002/pssa.2211090102.

[75] M. Mesrine, N. Grandjean, and J. Massies. "Efficiency of $\mathrm{NH}_{3}$ as nitrogen source for GaN molecular beam epitaxy". Applied Physics Letters 72 (1998), p. 350. DOI: 10.1063/1.120733.

[76] P. Tasker. "The stability of ionic crystal surfaces". Journal of Physics C: Solid State Physics 12.22 (1979), p. 4977. DOI: 10.1088/0022$3719 / 12 / 22 / 036$.

[77] J. Narayan and B. Larson. "Domain epitaxy: A unified paradigm for thin film growth". Journal of Applied Physics 93.1 (2003), pp. 278-285. DOI: $10.1063 / 1.1528301$.

[78] B.-J. Schäfer et al. "Arsenic passivation of MOMBE grown GaAs surfaces". Surface Science 204.3 (1988), pp. 485-490. DOI: 10.1016/00396028(88) 90228-2.

[79] U. Resch et al. "Arsenic passivation of MBE grown GaAs (100): structural and electronic properties of the decapped surfaces". Surface science 269 (1992), pp. 797-803. DOI: 10.1016/0039-6028(92)91351-B.

[80] L. H. Lewis and F. Jiménez-Villacorta. "Perspectives on permanent magnetic materials for energy conversion and power generation". Met- 
allurgical and Materials Transactions A 44.1 (2013), pp. 2-20. DOI: 10.1007/s11661-012-1278-2.

[81] W. Y. Dong et al. "Phase transitions in samarium at high pressures". Physical Review B 35 (3 Jan. 1987), pp. 966-970. DoI: $10.1103 /$ PhysRevB.35.966.

[82] C. Dufour et al. "Epitaxial growth of dhcp samarium: single crystal films and Sm/Nd superlattices". Journal of Crystal Growth 234.2 (2002), pp. 447-453. DOI: 10.1016/S0022-0248(01)01719-5.

[83] A. K. Burnham and G. T. Jameson. "Oxidation kinetics for thin rareearth metal films". Journal of Vacuum Science \& Technology A: Vacuum, Surfaces, and Films 5.4 (1987), pp. 1713-1716. DOI: 10.1116/1. 574515 .

[84] K. W. Kolasinski. Surface Science: foundations of catalysis and nanoscience. John Wiley \& Sons, Ltd, Apr. 2012. DOI: 10 . 1002 / 9781119941798.

[85] C. Alcock, V. Itkin, and M. Horrigan. "Vapour pressure equations for the metallic elements: 298-2500K". Canadian Metallurgical Quarterly 23.3 (1984), pp. 309-313. DOI: 10.1179/cmq.1984.23.3.309.

[86] N. Grandjean et al. "GaN evaporation in molecular-beam epitaxy environment". Applied Physics Letters 74.13 (1999), pp. 1854-1856. DOI: $10.1063 / 1.123691$.

[87] S. Fernández-Garrido et al. "In situ GaN decomposition analysis by quadrupole mass spectrometry and reflection high-energy electron diffraction". Journal of Applied Physics 104.3 (2008), p. 033541. DOI: $10.1063 / 1.2968442$.

[88] A. Stenborg et al. "Valence-transition-induced $5 \times 5$ surface reconstruction of Sm (0001)". Physical Review Letters 63.2 (1989), p. 187. DOI: 10.1103/PhysRevLett.63.187. 
[89] A. Feher and J. Dudáš. "ON INFLUENCE OF MAGNETIC STRUCTURE ON THE ELECTRIC CHARGE TRANSPORT IN SAMARIUM AND THULIUM THIN FILMS". Periodica Polytechnica Electrical Engineering 36.1 (1992), pp. 53-59.

[90] A. Rosengren and B. Johansson. "Valence instability of the samarium metal surface". Physical Review B 26.6 (1982), p. 3068. DOI: 10.1103/ PhysRevB.26.3068.

[91] E. Lundgren et al. "Geometry of the valence transition induced surface reconstruction of Sm (0001)". Physical Review Letters 88.13 (2002), p. 136102. DOI: $10.1103 /$ PhysRevLett.88.136102.

[92] J. Dai et al. "Restoring pristine $\mathrm{Bi}_{2} \mathrm{Se}_{3}$ surfaces with an effective Se decapping process". Nano Research 4.8 (2015), pp. 1222-1228. DOI: 10.1007/s12274-014-0607-8. 